\title{
Measurement of the Weak Mixing Angle in Møller Scattering*
}

\author{
Klejda Bega \\ Stanford Linear Accelerator Center \\ Stanford University \\ Stanford, CA 94309
}

SLAC-Report-739

Prepared for the Department of Energy under contract number DE-AC02-76SF00515

Printed in the United States of America. Available from the National Technical Information Service, U.S. Department of Commerce, 5285 Port Royal Road, Springfield, VA 22161.

* Ph.D. thesis, California Institute of Technology, Pasadena, CA 


\title{
Measurement of the Weak Mixing Angle in Møller Scattering
}

\author{
Thesis by \\ Klejda Bega \\ In Partial Fulfillment of the Requirements \\ for the Degree of \\ Doctor of Philosophy
}

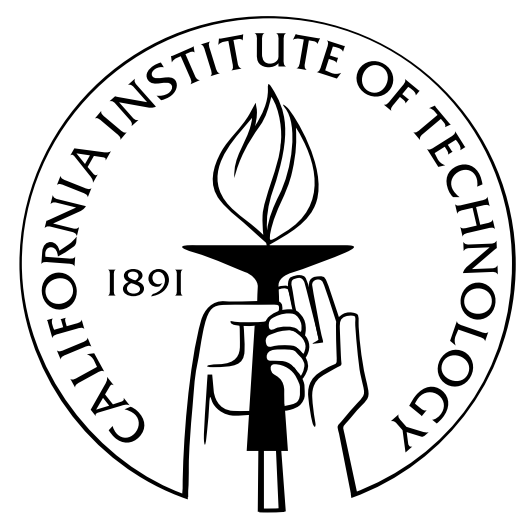

California Institute of Technology

Pasadena, California

2004

(Defended April 12, 2004) 
(C) 2004

Klejda Bega

All Rights Reserved 
Në mes tuaj kam qëndruar

E jam duke përvëluar, Që t'u ap pakëzë dritë, Natënë t'ua bënj ditë.

Do të tretem, të kullohem, Të digjem, të përvëlohem, Që t'u ndrinj mir' e të shihni,

Njëri-tjatërin të njihni.

Naim Frashëri, Fjalët e Qiririt

To my father, Adnan Bega (1944 - 1999) 


\section{Acknowledgements}

During graduate school, I had the opportunity to work with and be taught by a great group of people. First, I would like to acknowledge Emlyn Hughes, who for all practical purposes has been my advisor ever since I signed up for a summer project at the end of my freshman year at Caltech. His support, guidance, encouragement and savvy were instrumental throughout my student years. Furthermore, his openmindness and frankness allowed me to pursue alternative career options.

I was fortunate to be part of the last fixed target experiment at the Stanford Linear Accelerator Center. While there, I had many mentors who enabled me to learn and grow as a scientist, and without whom Experiment E158 would not have been a success. I will always be grateful to my "second advisor", Yury Kolomesky, for trusting me with beam monitoring and tirelessly answering all my questions. I have boundless appreciation for the help, advice and example set by Krishna Kumar, Paul Souder, Ray Arnold, Mike Woods, Ted Fieguth, Peter Bosted, Piotr Decowski, Steve Rock, Zen Szalata, Perry Anthony, John Weisend, Alexandre Deur and Dieter Walz. Many thanks go to Ken Baird, MO, Mike Racine, Terry Tuck and Carl Hudspeth for welcoming me to the End Station A and assisting with the alcove installation.

The experiment was at times stressful and the shifts were numerous but going through this experience with my "comrades in arms" made it so much easier. Mark J., Pete, Dave, Carlos, Antonin, Waled, Brian, Imran, Brock, Mark C., Baris and Lisa altruistically shared shifts, plots, code, life stories, laughs, junk food, and offered unlimited assistance. Guodong and Wenjin accommodated my return to Caltech and graciously allowed me take over the office. Georgia, whose expediency, dependability and care made coming to work enjoyable, deserves a special mention. 
I am very grateful to have had my life enriched by many close friends and family, who not only offered steady encouragement, but never allowed me to loose perspective. There are so many people to thank: Ganya and Yuri for their friendship, generosity and career advice; you guys are an inspiration to me. My gal pals Jasmine, Adrienne, Tina and Yue for San Francisco outings, fondue nights, insightful chats, goofiness, optimism and much needed female perspective. Anatole for his perceptive opinions and long distance phone calls. John for his open tab at Hailie's and for TiVo-ing my favorite show. Olta, Fiori, Frida and Erinda for continuing to be my good and trusted friends since elementary school and for making me an "auntie" several times now. My aunt Zana for her kindness, humor and for taking such good care of my grandma. I am particularly indebted to Mr. Christos Achis for his hospitality, wisdom and for teaching me a good lesson on how to separate facts from propaganda.

The last three years of graduate school would not have been as adventurous and fulfilling if it wasn't for Kaice. Without him I would have missed out on taekwondo, surfing, 50 miles/hour downhill skiing, stand-up comedy, the intricacies of moving cement blocks, being lost on a dingy boat in the middle of the Pacific, and eating "vegetarian" stuffed tomatoes in Greece.

Finally and above all, I want to thank my mother, Afërdita, whose unconditional love, unwavering support, selflessness and strength saw us through good times and bad. She never made me feel guilty about being absent from home for so long and always stood by my decisions. I feel privileged to be her daughter. 


\section{Abstract}

The weak mixing parameter, $\sin ^{2} \theta_{w}$, is one of the fundamental parameters of the Standard Model. Its tree-level value has been measured with high precision at energies near the $Z^{0}$ pole; however, due to radiative corrections at the one-loop level, the value of $\sin ^{2} \theta_{w}$ is expected to change with the interaction energy. As a result, a measurement of $\sin ^{2} \theta_{w}$ at low energy $\left(Q^{2} \ll m_{Z}\right.$, where $Q^{2}$ is the momentum transfer and $m_{Z}$ is the $Z$ boson mass), provides a test of the Standard Model at the one-loop level, and a probe for new physics beyond the Standard Model.

One way of obtaining $\sin ^{2} \theta_{w}$ at low energy is from measuring the left-right, parityviolating asymmetry in electron-electron (Møller) scattering: $A_{P V}=\frac{\sigma_{R}-\sigma_{L}}{\sigma_{R}+\sigma_{L}}$, where $\sigma_{R}$ and $\sigma_{L}$ are the cross sections for right- and left-handed incident electrons, respectively. The parity violating asymmetry is proportional to the pseudo-scalar weak neutral current coupling in Møller scattering, $g_{e e}$. At tree level $g_{e e}=\left(\frac{1}{4}-\sin ^{2} \theta_{w}\right)$. A precision measurement of the parity-violating asymmetry in Møller scattering was performed by Experiment E158 at the Stanford Linear Accelerator Center (SLAC). During the experiment, $\sim 50 \mathrm{GeV}$ longitudinally polarized electrons scattered off unpolarized atomic electrons in a liquid hydrogen target, corresponding to an average momentum transfer $Q^{2} \sim 0.03(\mathrm{GeV} / \mathrm{c})^{2}$. The tree-level prediction for $A_{P V}$ at such energy is $\simeq 300 \mathrm{ppb}$. However one-loop radiative corrections reduce its value by $\sim 40 \%$.

This document reports the E158 results from the 2002 data collection period. The parity-violating asymmetry was found to be $A_{P V}=-160 \pm 21$ (stat.) \pm 17 (syst.) ppb, which represents the first observation of a parity-violating asymmetry in Møller scattering. This value corresponds to a weak mixing angle at $Q^{2}=0.026(\mathrm{GeV} / \mathrm{c})^{2}$ of $\sin ^{2} \theta_{w \overline{M S}}=0.2379 \pm 0.0016$ (stat.) \pm 0.0013 (syst.), which is -0.3 standard deviations 
vii

away from the Standard Model prediction: $\sin ^{2} \theta_{w} \frac{\text { predicted }}{M S}=0.2385 \pm 0.0006$ (theory). The E158 measurement of $\sin ^{2} \theta_{w}$ at a precision of $\delta\left(\sin ^{2} \theta_{w}\right)=0.0020$ provides new physics sensitivity at the $\mathrm{TeV}$ scale. 


\section{Contents}

Acknowledgements $\quad$ iv

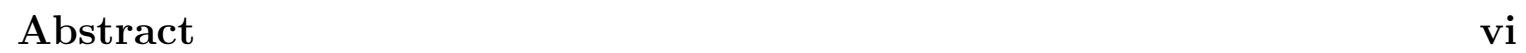

1 Theoretical Background 1

1.1 Introduction . . . . . . . . . . . . . . . . . . 1

1.2 Parity Violation in Møller scattering . . . . . . . . . . . . . . 4

1.2.1 One-Loop Electroweak Radiative Corrections . . . . . . . . . . 7

1.2.2 Sensitivity to New Physics . . . . . . . . . . . . . 8

1.3 Overview of the Experiment . . . . . . . . . . . . . . . 10

2 Polarized Electron Source and Feedbacks 13

2.1 The source . . . . . . . . . . . . . . . . . 13

2.1.1 The Laser System . . . . . . . . . . . . . . . . . 15

2.1.2 Diagnostics Bench ................ 17

2.1.3 Helicity Control Bench . . . . . . . . . . . . . . 18

2.1.4 Cathode Diagnostics Bench . . . . . . . . . . . . . 19

2.1.5 The Cathode ..................... 19

2.2 The Feedback System . . . . . . . . . . . . . . . . . . . . . . . 20

2.2.1 Helicity Sequence . . . . . . . . . . . . . . . . 21

2.2.2 Active Feedback Loops and Feedback Algorithm . . . . . . . . 22

3 Precision Beam Monitoring $\quad 25$

3.1 Charge Monitors . . . . . . . . . . . . . . 26 
3.2 Beam Position Monitors . . . . . . . . . . . . . . . 28

3.2.1 Theoretical Description of Resonant Cavities . . . . . . . . . . 29

3.2.1.1 Excitation by Gaussian Bunch . . . . . . . . . 30

3.2.1.2 Excitation by a Bunch Train . . . . . . . . . . 31

3.2.1.3 Detuning from Resonance . . . . . . . . . . . 32

3.2.2 Mechanical Design . . . . . . . . . . . . . 32

3.2 .3 Electronics . . . . . . . . . . . . . . . . . . 35

3.2.4 Position Measurement . . . . . . . . . . . . . . 37

3.2.5 Results........................ 40

3.3 Wire Array . . . . . . . . . . . . . . . . . . . . . . . . . 41

3.4 Synchrotron Light Monitor . . . . . . . . . . . . . . . . . . . . 42

4 Target and the Spectrometer $\quad 44$

4.1 Liquid Hydrogen Target . . . . . . . . . . . . . . . . 45

4.1.1 Foil Target. . . . . . . . . . . . . . . 47

4.2 E158 Spectrometer . . . . . . . . . . . . . . . 48

4.2.1 Dipole Chicane . . . . . . . . . . . . . . . . 49

4.2.2 Photon Collimators . . . . . . . . . . . . . 50

4.2 .3 Momentum Collimator . . . . . . . . . . . . . . . . . 51

4.2.4 "Holey" Collimator . . . . . . . . . . . . . . . 52

4.2.5 Quadrupoles................... 53

4.2.6 Synchrotron Collimators and Collimator Masks . . . . . . . 54

5 Detectors $\quad 57$

5.1 Profile Detector . . . . . . . . . . . . . . . . . 57

5.2 The Møller and the ep Detector . . . . . . . . . . . . . . 60

5.2 .1 Detector Geometry . . . . . . . . . . . . . 60

5.2.2 Detector Electronics . . . . . . . . . . . . . . . 62

5.3 Pion Detector ........................ 64

5.4 Polarimeter Detector . . . . . . . . . . . . . . . . 65

5.5 Luminosity Monitor . . . . . . . . . . . . . . . . . . . 68 
5.6 Good Spill and Bad Spill Monitors . . . . . . . . . . . . . . . 69

6 Analysis and Results $\quad 70$

6.1 The Møller Detector Analysis . . . . . . . . . . . . . . . 70

6.1.1 Calculating the Raw Asymmetry . . . . . . . . . . . . 70

6.1.2 Removing Beam Helicity Correlations . . . . . . . . . . . . . . 71

6.1.2.1 Regression................. 71

6.1.2.2 Dithering ..................... 73

6.1.3 Calculating the Overall Asymmetry . . . . . . . . . . . . 74

6.1.4 Blind Analysis . . . . . . . . . . . . . . . 75

6.1.5 Analysis Data Selection . . . . . . . . . . . 75

6.1.5.1 Baseline Cuts .............. 76

6.1.5.2 Reducing Systematic Effects . . . . . . . . . 77

6.2 The Møller Detector Asymmetry . . . . . . . . . . . . . . 78

6.3 Corrections and Dilution Factors . . . . . . . . . . . . . 81

6.3.1 Beam Systematic Uncertainties . . . . . . . . . . . 82

6.3.1.1 Systematic Uncertainties from Beam Spot Size Asymmetries ................. 85

6.3.2 Electron-Proton Background . . . . . . . . . . . . 85

6.3.3 Pion Corrections . . . . . . . . . . . . . . . 87

6.3.4 Corrections due to Neutral Backgrounds . . . . . . . . . . . 87

6.3.5 Linearity of the Møller Detector Response . . . . . . . . . . . 88

6.3.6 Beam Polarization ................. 89

6.3.7 Luminosity Monitor Results . . . . . . . . . . . . . . . . 90

6.4 The Parity-Violating Asymmetry . . . . . . . . . . . . . . . 91

7 The Weak Mixing Angle and Final Conclusions 95

7.1 Calculating the Weak Mixing Angle . . . . . . . . . . . . 95

7.1 .1 New Physics Limits . . . . . . . . . . . . . . . 99

7.2 Future Experiments . . . . . . . . . . . . . . . . . 100

7.3 Conclusions . . . . . . . . . . . . . . . . . 105 


\section{List of Figures}

1.1 Measurements of $\sin ^{2} \theta_{w}$ as a function of momentum transfer $Q$. The solid line and the dotted line are the theoretical prediction at high $Q$ and low $Q$, respectively. PDG 2002 refers to the 2002 result reported by the Particle Data Group, and corresponds to an average of the SLD and LEP measurements. . . . . . . . . . . . . . . . . .

1.2 Tree level Feynman diagrams for Møller scattering, representing the electromagnetic and weak interactions. . . . . . . . . . . . 5

1.3 Kinematics for Møller scattering in the center of mass frame. . . . . . . 6

1.4 The primary one-loop contributions to $A_{P V}: \gamma-Z$ mixing diagrams (a-c) and the W-loop contribution to the anapole moment $(\mathrm{d}) . \quad$. . . 8

1.5 Box diagrams with two heavy bosons. . . . . . . . . . . . . 8

1.6 One-photon vertex and the Z-loop contribution to the anapole moment. 8

1.7 Schematic of the E158 experimental design. . . . . . . . . . . . . 11

1.8 (a) The parity-violating asymmetry and the differential scattering cross section as a function of $\left|\cos \Theta_{c m}\right|$, where $\Theta_{c m}$ is the scattering angle in the center of mass frame. (b) The figure of merit (f.o.m) as a function of $\left|\cos \Theta_{c m}\right| \ldots \ldots \ldots \ldots \ldots$. . . . . . . . . . . . . . . . . 12

2.1 Overview of E158 polarized source. . . . . . . . . . . . . . 14

2.2 Laser intensity and the percent jitter as a function of time within the pulse. The SLICE Pockels cell selects a 270 ns section at the region with the lowest jitter. . . . . . . . . . . . . . . . 16

2.3 Temporal profile of the laser pulse. . . . . . . . . . . . . 16

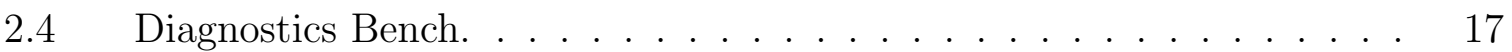


2.5 Definition of right- and left-helicity for the electron beam (top) and the laser beam polarization (bottom) . . . . . . . . . . . . . 18

2.6 Gradient-doped strained GaAs cathode. . . . . . . . . . . . . 20

2.7 Band-gap diagram for GaAs. . . . . . . . . . . . . . . . . 21

2.8 Feedback performance during Run I. The dotted line corresponds to $\frac{1}{\sqrt{N}}$ statistical scaling. (a) Plot of the integrated charge asymmetry in parts per million (ppm). (b) Integrated energy asymmetry in keV. (c)

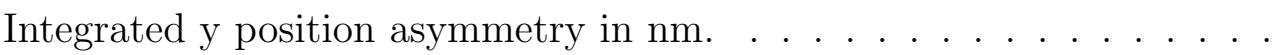

3.1 Overview of the SLAC 2 mile accelerator, A-Line and End Station A. . 26

3.2 Mechanical design of E158 toroids. . . . . . . . . . . . . . . 27

3.3 Toroid resolution for one run (given by the root mean square), using two of the ESA toroids. . . . . . . . . . . . . . . . . 28

3.4 Dynamic variables of a cavity coupled to a waveguide and the beam. . 29

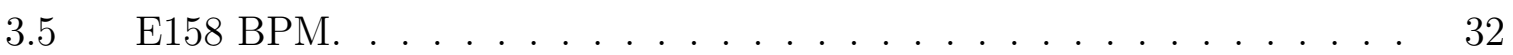

$3.6 \phi$-cavity dimensions. . . . . . . . . . . . . . . . . . 33

$3.7 \quad$ x-cavity dimensions. . . . . . . . . . . . . . . . . . . . 34

3.8 Cavity setup. . . . . . . . . . . . . . . . . . 34

3.9 Power distribution chassis. . . . . . . . . . . . . . . . . . 36

3.10 BPM processor. . . . . . . . . . . . . . 36

3.11 On the left: $I F_{1}$ and $I F_{2}$ waveforms after phase adjusts. On the right:

Fitting $A \cdot e^{-\frac{\pi \cdot 2856 \cdot 10^{6}}{Q_{L}} t}$ to the exponentially decaying part of $I F_{1}$ waveform. 38

3.12 BPM placement along the beam line. . . . . . . . . . . . . . . 39

3.13 Resolution and agreement in $\mathrm{y}$ for the BPM triplet. The spread in the linear plot is due to beam jitter. A Gaussian fit of the residuals determines the BPM resolution and agreement. . . . . . . . . . . . 40

3.14 Wire array display when averaged over 1 second. . . . . . . . . . . . . 42

3.15 Top view schematic of the Synchrotron Light Monitor. . . . . . . . . . 43

4.1 The layout of End Station A. . . . . . . . . . . . . . . . 44 
xiv

4.2 The liquid hydrogen target. (a) Photograph of the target cell and the pump. (b) Schematic of the target loop. (c) Photograph of the scattering chamber. (d) Photograph of the wire mesh disks. . . . . . . . . . . . 46

4.3 The orientation of the polarimetry foils with respect to the beam. . . . 47

4.4 Top view schematic of the spectrometer and detector layout. The scale along the $\mathrm{z}$ direction is in meters, while the scale along the $\mathrm{x}$ is in centimeters. . . . . . . . . . . . . . . . . . 48

4.5 AutoCAD rendering of the first photon collimator. . . . . . . . . . 50

4.6 (a) Photograph of the momentum collimator. (b) Upstream and downstream dimensions of the momentum collimator. . . . . . . . . . . . 51

4.7 Simulations of the phase space of the Møller and ep flux at the Møller detector. (a) Without the momentum collimator. (b) With the momentum collimator. . . . . . . . . . . . . . . . .

4.8 Photograph of the "holey" collimator 3QC1A (front) and the momentum collimator 3QC1B (back). . . . . . . . . . . . . . . 53

4.9 Scans of the flux profile at the detector with the quadrupoles on and off. The points represent the data scan and the open histogram is the Monte Carlo simulation. The Møller contribution is represented by a shaded histogram and the ep contribution by a hatched one. The parityviolating asymmetry was measured for regions I and III. . . . . . . . . 54

4.10 Schematic of the drift pipe containing the synchrotron collimators and the collimator masks. . . . . . . . . . . . . . . . .

5.1 The E158 detectors. Top: schematic of the side view of the bottom half of the detector cart. Bottom: a 3-D rendering of the detector cart. All detectors had cylindrical symmetry. Note that the polarimetry detector is not shown, but was mounted on the back and to the right of the profile detector when looking downstream. . . . . . . . . . . . . 
5.2 Schematic of the profile detector. One can see the four Cerenkov counters mounted to the wheel. Depicted in darker blue on the left figure is the cable used to rotate the wheel. . . . . . . . . . . . . . . 59

5.3 Schematic of a Cerenkov counter and the moving mechanism. . . . . . 60

5.4 A photograph of the Møller and ep detector as the ep ring was being assembled. The lightguides would be attached to the fibers protruding out of the copper slabs. . . . . . . . . . . . . . . . . 61

5.5 (a) Schematic of the Møller detector. Quartz fibers are sandwiched between copper slabs. (b) Schematic of the the orientation of copper slabs and fibers with respect to the incident beam. The copper-fibercopper wedges are separate for the Møller and ep rings. Also shown are the outer diameters for each ring. . . . . . . . . . . . . . . . 62

5.6 Schematic of the Møller and ep detector electronics. . . . . . . . . . . .

5.7 Plot of the square of the experimental asymmetry width $\left(\sigma_{e x p}^{2}\right)$ versus the inverse of beam intensity ( $1 / \mathrm{N}, \mathrm{N}=$ number of electrons.) The Møller detector resolution is given by the square root of the offset: $\sqrt{12000 \mathrm{ppm}^{2}}$

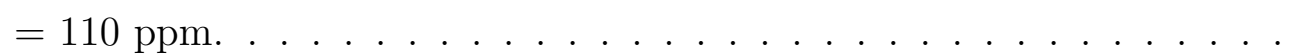

5.8 Schematic of the Pion detector (in red) sitting behind shielding and the Møller and ep detector. . . . . . . . . . . . . . . . . 65

5.9 Schematic of the polarimeter detector. . . . . . . . . . . . . 67

5.10 (a) Diagram of the luminosity monitor chambers. (b) Photograph of the luminosity monitor. . . . . . . . . . . . . . . .

6.1 (a) The raw asymmetry of a single channel taken over one hundred thousand pulse pairs, namely, two hundred thousand pulses. (b) The regressed asymmetry of the same channel. Note how the RMS has been reduced by more than a factor of two. (c) Raw channel asymmetry versus position asymmetry. (d) Same plot as in (c), except that the channel asymmetry is now regressed out. The first-order position dependence has been removed. . . . . . . . . . . . . . . . . . 
6.2 Møller asymmetry versus run and versus slug during Run II. The average asymmetries are obtained from fitting a zeroth degree polynomial to each plot. . . . . . . . . . . . . . . . . .

6.3 Plot of the Møller detector asymmetry for the four different sign-flip combinations and the overall average for both Run I and Run II. The asymmetries have been corrected, such that they all have the same sign.

6.4 The Møller asymmetry per channel versus channel (azimuth) for the inner ring. . . . . . . . . . . . . . . . . .

6.5 A profile detector scan taken with the "holey" collimator inserted. Also shown is the Monte Carlo simulation, normalized to the data sample. .

6.6 A Monte Carlo simulation of the expected asymmetry at the luminosity monitor. Contributions from the Møller and ep electrons, and from electrons scattering off the target aluminum windows (eA Elastic) are shown together with their total sum. . . . . . . . . . .

6.7 Luminosity monitor asymmetry for the four different spin-flip combinations and the overall average for Run I and Run II. The plots demonstrate how averaging over asymmetry reversals suppressed systematic effects. . . . . . . . . . . . . . . . .

6.8 The parity violating asymmetry for each slug for both Run I and Run II. The data has not been corrected for asymmetry sign flips and the pink solid line represents the grand average with the expected asymmetry sign for each sign flip configuration. The uncertainty shown is only the statistical uncertainty. . . . . . . . . . . . . .

7.1 Bremsstrahlung contributions to the analyzing power. . . . . . . . . 96

7.2 Measurements of the weak mixing angle at the $Z^{0}$ pole by the atomic parity violation experiment (Qw), NuTeV experiment, E158 Experiment, and the LEP and SLD experiments (PDG2002) . . . . . . . . . . 
xvii

7.3 Measurements of $\sin ^{2} \theta_{w}$ as a function of momentum transfer $Q$. The solid line and the dotted line are the theoretical prediction at high $Q$ and low $Q$, respectively. . . . . . . . . . . . . . 


\section{List of Tables}

$2.1 \quad$ Laser pulse properties. . . . . . . . . . . . . . . . . 15

$3.1 \quad$ BPM resolutions per pulse during Run I and Run II. . . . . . . . . . . 41

3.2 BPM agreement averaged over Run I and Run II. . . . . . . . . . . . . 41

6.1 List of systematic cuts and their cumulative acceptance for both Run I and Run II. . . . . . . . . . . . . . . . . . . . . . . . . 78

6.2 Various ring combinations used to study first-order beam systematic uncertainties for each beam parameter. . . . . . . . . . . . . . 83

6.3 Total (elastic+inelastic) ep asymmetry corrections and dilution factors. 86

6.4 Corrections and dilution factors due to neutral backgrounds. . . . . . . 88

6.5 Corrections and dilution factors for Run I and Run II. . . . . . . . . . 92

7.1 Analyzing powers for Run I and Run II given for each energy, and averaged over an entire Run. . . . . . . . . . . . . . . . . . . 96

7.2 The weak charge and weak mixing angle measured at $Q^{2}=0.026$ $(\mathrm{GeV} / \mathrm{c})^{2}$ during Run I and Run II. The results are -0.6 and +0.3 standard deviations away from the Standard Model prediction for Run I and Run II, respectively. . . . . . . . . . . . . . . . . . . . . . . . . 98 


\section{Chapter 1}

\section{Theoretical Background}

\section{$1.1 \quad$ Introduction}

One of the greatest achievements of physics in the twentieth century was the formulation of the Standard Model. Incorporating quantum electrodynamics, quantum chromodynamics and the electroweak theory, the Standard Model successfully described all the interactions among elementary particles, except for gravity. One of the cornerstones of the Standard Model is the electroweak theory, which unifies electromagnetic and weak interactions. Although seemingly of a different nature (massless mediator for electromagnetic interactions versus massive mediators for weak interactions), the electroweak theory incorporates these interactions into a single mathematical framework.

The electroweak theory was formulated by Glashow, Weinberg and Salam. In 1961 Glashow proposed a model where he unified the electromagnetic and weak interactions [23]. The model postulated the existence of weak neutral currents and, at that point, only charged weak interactions, mediated by $W^{+}$and $W^{-}$, had been observed. In 1967, Weinberg and Salam took Glashow's model one step further [65] [53], formulating it as a gauge theory with "spontaneous symmetry breaking" ${ }^{1}$. In 1971, 't Hooft showed that the Glashow-Weinberg-Salam scheme (GWS) was renormaliz-

\footnotetext{
${ }^{1}$ Spontaneous symmetry breaking occurs when the ground state of a system is asymmetric with respect to the symmetries that govern its dynamics. In the electroweak theory the weak and electromagnetic interactions are symmetries of the Lagrangian, but are broken by the vacuum (the ground state). The spontaneous symmetry breaking requires gauge vector bosons to acquire mass, which is known as the Higgs mechanism.
} 
able [62] [61]. The discovery of weak neutral currents in 1973 [29] provided evidence for the GWS scheme. However, this was not the only electroweak unification model proposed at the time. What set it apart from other models was its $\mathrm{SU}(2)_{L} \times \mathrm{U}(1)_{Y}$ symmetry. Among the implications was that the weak interactions do not conserve parity.

The confirmation for the GWS model arrived in 1978, when SLAC Experiment E122 measured a parity-violating asymmetry $A_{P V}=\left(-9.5 \times 10^{-5}\right) Q^{2}(\mathrm{GeV} / \mathrm{c})^{-2}$ in the cross section of scattering longitudinally polarized electrons off a deuteron target [49]. The asymmetry was used to determine the weak mixing angle $\sin ^{2} \theta_{w}$, a fundamental parameter of the Standard Model, which correlates the weak coupling constants $\mathrm{g}_{w}$ and $\mathrm{g}_{z}$ to the electromagnetic coupling constant $\mathrm{g}_{e}$ :

$$
\mathrm{g}_{\mathrm{w}}=\frac{\mathrm{g}_{\mathrm{e}}}{\sin \theta_{w}} \quad, \quad \mathrm{~g}_{\mathrm{z}}=\frac{\mathrm{g}_{\mathrm{e}}}{\cos \theta_{w} \sin \theta_{w}}
$$

The weak mixing angle found by the E122 experiment was $\sin ^{2} \theta_{w}=0.224 \pm 0.020$,

The parity violation measured by Experiment E122 was consistent with the GWS model and the value found for the weak mixing angle was consistent with results from previous neutrino-nucleon experiments [50]. On the other hand, the E122 results ruled out gauge theories which predicted no parity violation. Moreover, the E122 results ruled out "hybrid" models, which assigned right-handed electrons to a doublet and right-handed quarks to a singlet. The "hybrid" models had not been excluded by the neutrino-nucleon experiments, however they did not agree with the E122 data.

The establishment of the GWS electroweak theory necessitated the task of measuring to high precision the electroweak parameters, such as the weak mixing angle, as means of testing the Standard Model. One way to measure the weak mixing angle with high precision was to conduct experiments at energies near the $\mathrm{Z}^{0}$ pole, where the weak interactions dominate over the electromagnetic interactions. The LEP [60] and SLD collaborations [58] ascertained the weak mixing angle by measuring leftright and forward-backward asymmetries from electron-positron collisions near the $\mathrm{Z}^{0}$ pole. The results were in excellent agreement with the Standard Model. However, one-loop radiative corrections to the tree level diagrams introduce an energy depen- 
dence to the value of $\sin ^{2} \theta_{w}$. As a result, it becomes worthwhile to measure $\sin ^{2} \theta_{w}$ at different energy scales. These measurements not only provide important tests for the Standard Model, but also shed some light to possible new physics phenomena beyond the Standard Model.

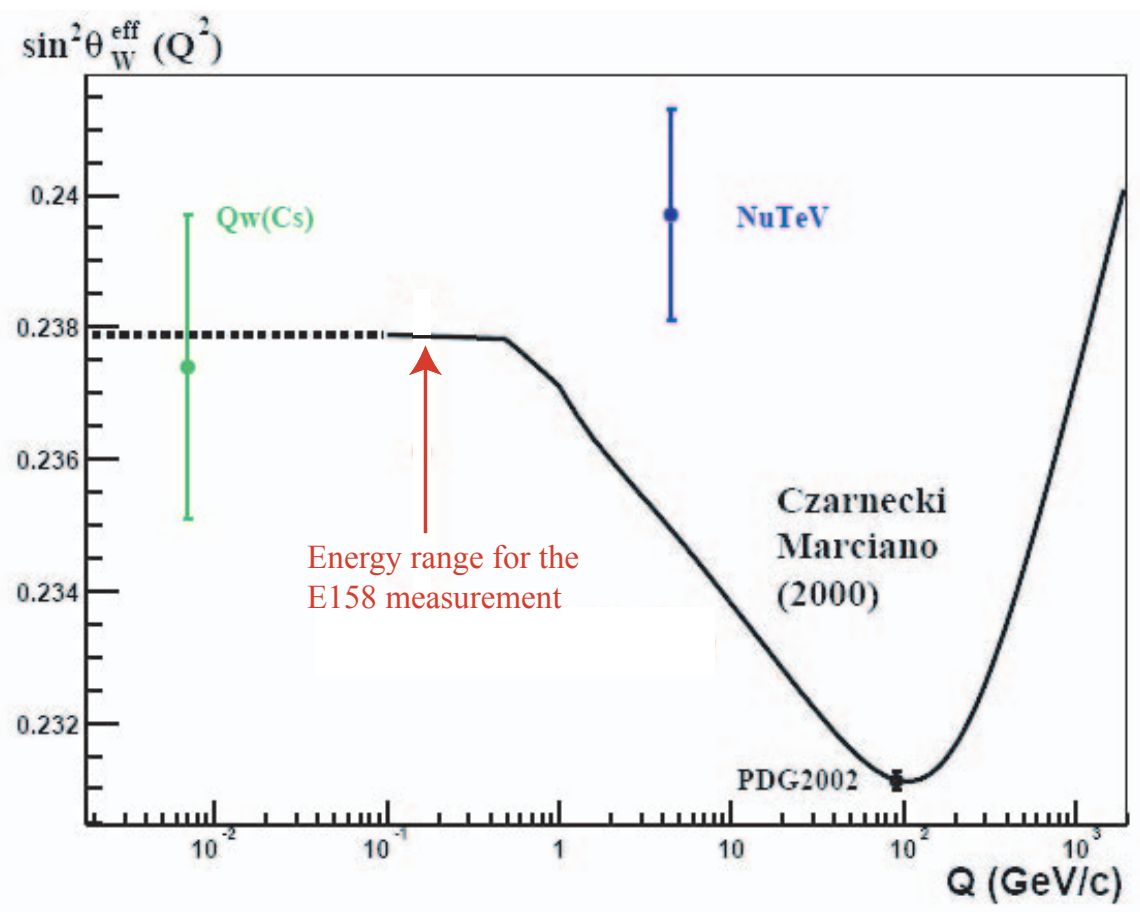

Figure 1.1: Measurements of $\sin ^{2} \theta_{w}$ as a function of momentum transfer $Q$. The solid line and the dotted line are the theoretical prediction at high $Q$ and low $Q$, respectively. PDG 2002 refers to the 2002 result reported by the Particle Data Group, and corresponds to an average of the SLD and LEP measurements.

Two experiments have been conducted at much lower energies. The NuTeV experiment [59] at Fermi National Accelerator Laboratory (FNAL) deduced $\sin ^{2} \theta_{w}$ from neutrino-nucleon deep inelastic scattering at an energy scale of $Q^{2} \sim 20(\mathrm{GeV} / \mathrm{c})^{2}$. The value reported by the $\mathrm{NuTeV}$ Collaboration is three standard deviations above the Standard Model prediction (Figure 1.1). There have been several attempts to explain the cause of this discrepancy such as nuclear effects in the iron target [44], or new physics effects including extra U(1) gauge bosons, new couplings, etc. [15]. Recent calculations of the $\mathcal{O}(\alpha)$ electroweak radiative corrections to deep-inelastic 
neutrino scattering suggest that radiative corrections can introduce theoretical uncertainties large enough to account for the $3 \sigma$ difference between the NuTeV result and the theoretical prediction [18].

The second experiment conducted at University of Colorado measured atomic parity violation for the $6 \mathrm{~S} \rightarrow 7 \mathrm{~S}$ transition in cesium [7] [16], probing at the $Q^{2} \sim 10^{-4}$ $(\mathrm{GeV} / \mathrm{c})^{2}$ energy scale. The experiment has a large theoretical uncertainty (Figure 1.1), rising from the complexity in determining the electronic wavefunction in heavy atoms, such as cesium. Figure 1.1 depicts measurements of $\sin ^{2} \theta_{w}$ as a function of $Q^{2}$ and the theoretical prediction of the running of $\sin ^{2} \theta_{w}$, as estimated by Czarnecki and Marciano [14]. From Figure 1.1 it is apparent that another measurement of the weak mixing angle at low energy would be useful in order to verify the Standard Model prediction for the energy dependence of $\sin ^{2} \theta_{w}$.

The goal of determining the weak mixing angle at low energy is fulfilled by SLAC Experiment E158. The experiment allows one to obtain the weak mixing angle from the parity-violating asymmetry in electron-electron (Møller scattering) at an average of $Q^{2} \sim 0.03(\mathrm{GeV} / \mathrm{c})^{2}$ and at a precision of $\delta\left(\sin ^{2} \theta_{w}\right) \sim 0.001$. The measurement is the first determination of parity non-conservation in Møller scattering. The E158 results offer unique sensitivity to new physics beyond the Standard Model at the TeV scale, as discussed in Section 1.2.2.

During the experiment, the parity-violating asymmetry was measured by scattering longitudinally polarized electrons off atomic electrons in a liquid hydrogen target. The polarized electrons were obtained from a 45.0 and $48.3 \mathrm{GeV}$ polarized electron beam at the Stanford Linear Accelerator Center (SLAC). This document contains a description of the E158 experimental design and analysis method, concluding with the results from the 2002 data collection periods.

\subsection{Parity Violation in Møller scattering}

Two electrons can interact electromagnetically, which conserves parity, or weakly, which violates parity. The scattering cross section of polarized electrons scattering 
off unpolarized electrons is spin dependent, due to the interference between the electromagnetic and weak interactions. Specifically, if left- and right-handed polarized incident electrons are used, there will be a non-zero left-right, parity-violating asymmetry $\left(A_{P V}\right)$ in the scattering cross section defined as

$$
A_{P V}=\frac{\sigma_{R}-\sigma_{L}}{\sigma_{R}+\sigma_{L}}
$$

where $\sigma_{R}$ and $\sigma_{L}$ are the scattering cross sections, integrated over the entire azimuth, for incident right- and left-handed electrons, respectively.
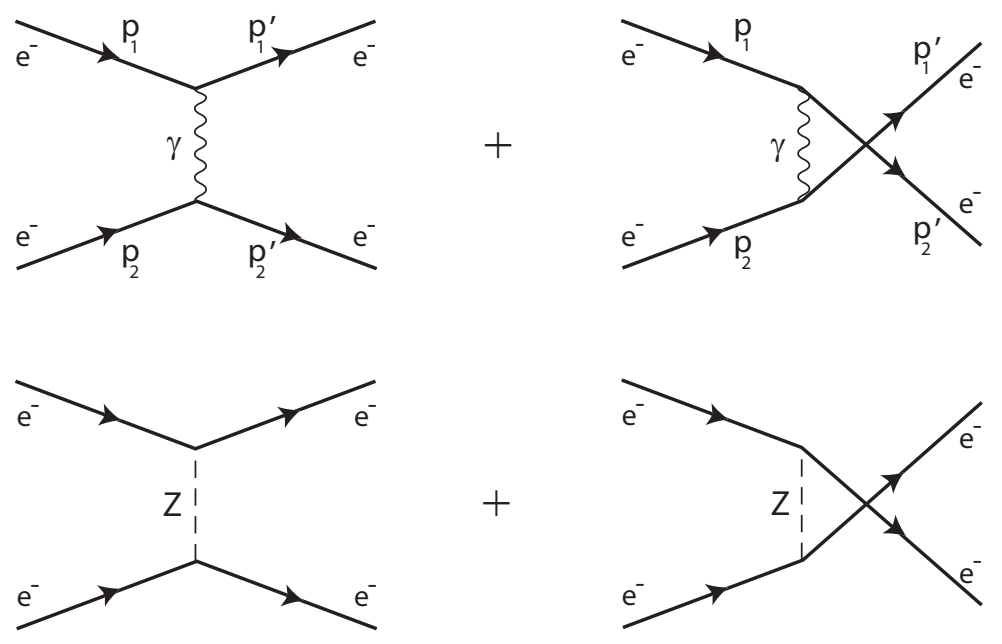

Figure 1.2: Tree level Feynman diagrams for Møller scattering, representing the electromagnetic and weak interactions.

The tree level Feynman diagrams for Møller scattering are shown in Figure 1.2, in which the interacting electrons exchange a photon or a $Z^{0}$. Since the electrons are indistinguishable, the crossed diagrams are also included. The spin-averaged differential scattering cross section for electron-electron scattering is given by

$$
\frac{d \sigma}{d \Omega}=\frac{\alpha^{2}}{2 m E} \frac{\left(3+\cos ^{2} \Theta_{c m}\right)^{2}}{\sin ^{4} \Theta_{c m}},
$$


where $\alpha$ is the fine structure constant, $m$ is the electron mass, $E$ is the incident beam energy, and $\Theta_{c m}$ is the scattering angle in the center of mass frame, shown in Figure 1.3.

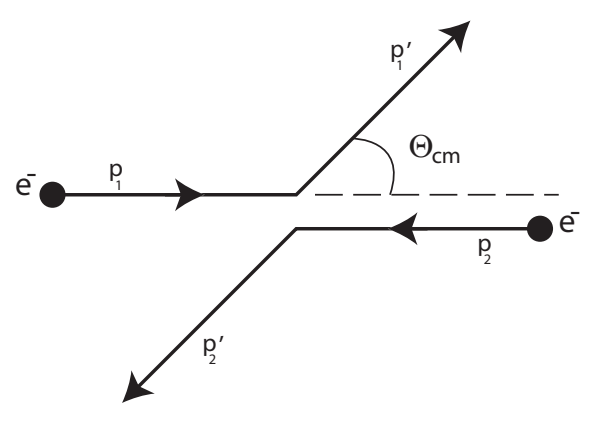

Figure 1.3: Kinematics for Møller scattering in the center of mass frame.

At tree level, the parity-violating asymmetry $A_{P V}$ is given by [17]

$$
A_{P V}=m E \frac{G_{F}}{\sqrt{2} \pi \alpha} \frac{16 \sin ^{2} \Theta_{c m}}{\left(3+\cos ^{2} \Theta_{c m}\right)^{2}}\left(\frac{1}{4}-\sin ^{2} \theta_{w}\right),
$$

where $G_{F}$ is the Fermi coupling constant. For SLAC Experiment E158, $E \approx 50 \mathrm{GeV}$, which corresponds to $Q^{2} \sim 0.03(\mathrm{GeV} / \mathrm{c})^{2}$. The expression in Eq. 1.3 is maximal when $\Theta_{c m}=90^{\circ}$. At this angle, assuming $100 \%$ beam polarization, and employing the $\mathrm{Z}$ pole value of $\sin ^{2} \theta_{w}=0.23$, the tree level prediction for the parity-violating asymmetry $A_{P V}$ is $\sim 300$ parts per billion (ppb). However, as it will be shown in Section 1.2.1, radiative corrections reduce $A_{P V}$ by $\sim 40 \%$ [14] to $\sim 180 \mathrm{ppb}$.

From Eq. 1.3 one can estimate the sensitivity of $A_{P V}$ to small changes in $\sin ^{2} \theta_{w}$ :

$$
\frac{\delta \sin ^{2} \theta_{w}}{\sin ^{2} \theta_{w}} \simeq-\frac{1-4 \sin ^{2} \theta_{w}}{4 \sin ^{2} \theta_{w}} \frac{\delta A_{P V}}{A_{P V}} .
$$

Since the value of the weak mixing angle is close to 0.25 , there is an enhanced sensitivity to small changes in $\sin ^{2} \theta_{w}$. Plugging into Eq. 1.4 the Standard Model prediction for the weak mixing angle at $Q^{2} \sim 0.03(\mathrm{GeV} / \mathrm{c})^{2}, \sin ^{2} \theta_{w}=0.238$ (Figure 1.1), 
one finds

$$
\frac{\delta \sin ^{2} \theta_{w}}{\sin ^{2} \theta_{w}} \simeq-0.05 \frac{\delta A_{P V}}{A_{P V}}
$$

Eq. 1.5 shows that a measurement of the parity-violating asymmetry with a precision of $\frac{\delta A_{P V}}{A_{P V}}=0.1$ leads to measurement of the weak mixing angle with a precision of $\frac{\delta \sin ^{2} \theta_{w}}{\sin ^{2} \theta_{w}}=0.005$, that is $\delta\left(\sin ^{2} \theta_{w}\right) \sim 0.001$.

\subsubsection{One-Loop Electroweak Radiative Corrections}

Møller scattering is a leptonic process. Therefore, one-loop radiative corrections can be calculated to high precision. For the E158 energy scale, the one-loop electroweak radiative corrections to $A_{P V}$ have been estimated by Czarnecki and Marciano [14]. The largest contributions to $A_{P V}$ come from the $\gamma-Z$ mixing and anapole moment ${ }^{2}$ diagrams shown in Figure 1.4. They modify the tree level $\left(\frac{1}{4}-\sin ^{2} \theta_{w}\right)$ in Eq. 1.3 by

$$
\frac{1}{4}-\kappa(0) \sin ^{2} \theta_{w}\left(m_{z}\right) \overline{M S}
$$

where $\sin ^{2} \theta_{w}\left(m_{z}\right)_{\overline{M S}}$ is the weak mixing angle using the modified minimal subtraction scheme $(\overline{M S})$ defined at an energy scale of $m_{Z}$, the $Z$ boson mass. The $\kappa(0)$ modification factor has been calculated to be

$$
\kappa(0)=1.0301 \pm 0.0025
$$

which represents a $3 \%$ increase in the value of $\sin ^{2} \theta_{w}$ from its $Z^{0}$ pole value. This result accounts for the running of $\sin ^{2} \theta_{w}$ as one moves from energies $Q^{2} \sim m_{Z}^{2}$ to the low $Q^{2}$ appropriate for experiment E158. The $3 \%$ shift in $\sin ^{2} \theta_{w}$ corresponds to a $38 \%$ reduction in $A_{P V}$.

The next largest radiative corrections to $A_{P V}$ come from heavy boson box diagrams and photonic vertex and box diagrams (Figures 1.5 and 1.6). However, the contribution to $A_{P V}$ is only at the few percent level. Taken together, all one-loop ra-

\footnotetext{
${ }^{2}$ The parity-violating coupling of an electron to an external electromagnetic field is defined as the electron anapole moment.
} 


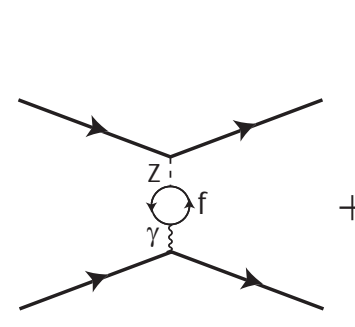

(a)

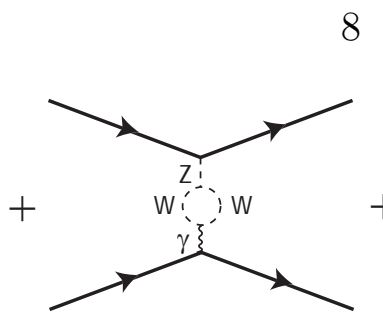

(b)

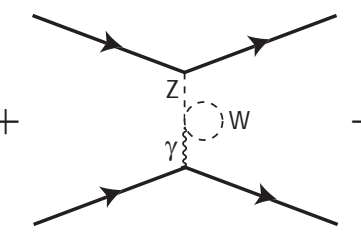

(c)

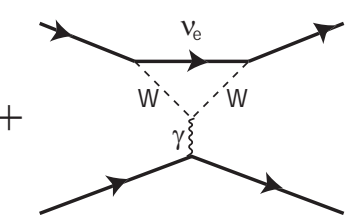

(d)

+ inverted + crossed diagrams

Figure 1.4: The primary one-loop contributions to $A_{P V}: \gamma-Z$ mixing diagrams (a-c) and the W-loop contribution to the anapole moment (d).

diative corrections contribute a $40 \pm 3 \%$ reduction in the asymmetry. Although these reductions increase the difficulty in measuring $A_{P V}$ - a very small quantity already - they do have the beneficial effect of making the the E158 result more sensitive to $\sin ^{2} \theta_{w}$ and "new physics" phenomena.

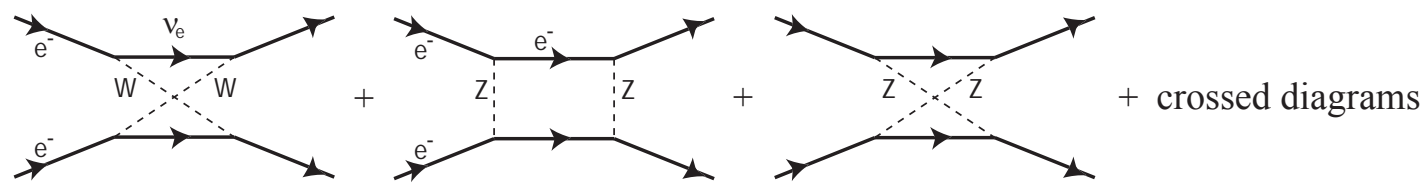

Figure 1.5: Box diagrams with two heavy bosons.

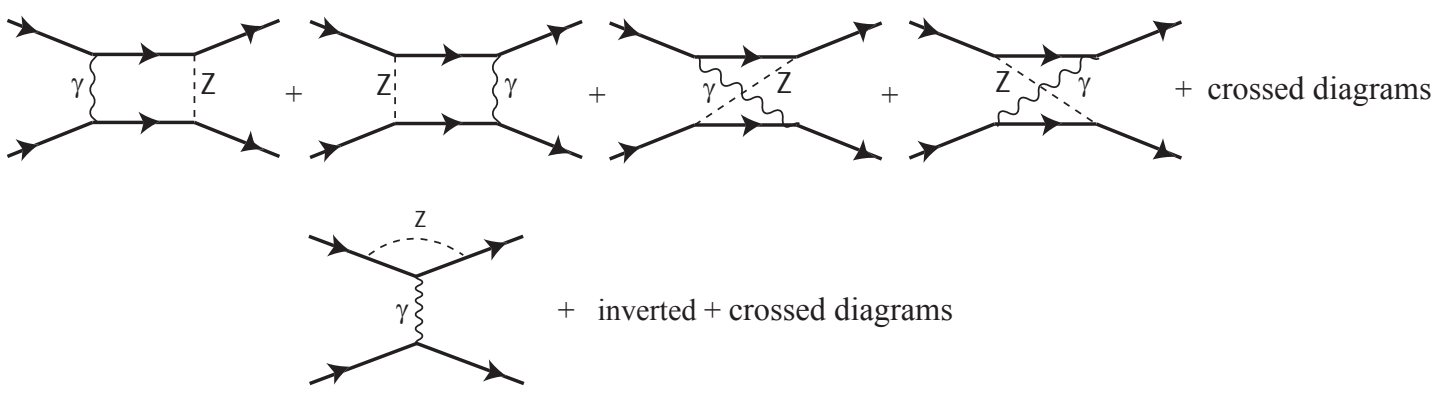

Figure 1.6: One-photon vertex and the Z-loop contribution to the anapole moment.

\subsubsection{Sensitivity to New Physics}

Measuring the weak mixing angle at an energy far from the $Z^{0}$ resonance with a precision of $\delta\left(\sin ^{2} \theta_{w}\right) \sim 0.001$, not only provides an important test of the Standard 
Model, but allows for exploration of new physics effects that may manifest themselves at the one-loop level. For these effects to be noticed, they have to contribute to the parity-violating $e^{-} e^{-} \longrightarrow e^{-} e^{-}$amplitude. Clearly, the single E158 measurement of $A_{P V}$ can not specify the exact source from the various "new physics" possibilities. However the magnitude and the sign of the deviation of $A_{P V}$ from the Standard Model prediction can discriminate among potential new physics models and provide useful limits. Furthermore, the E158 result is complementary to high energy collider experiments, which are sensitive to $L L+R R$ interactions, whereas E158 is sensitive to $L L-R R$ interactions. Examples of new physics scenarios that effect the E158 result include additional $Z^{\prime}$ bosons, new contact interactions and oblique corrections, all of which will be discussed briefly.

The presence of new neutral gauge bosons $Z^{\prime}$ at the $\mathrm{TeV}$ scale is predicted by several extensions of the Standard Model. The effect of these bosons to the parityviolating weak neutral current measured by the E158 experiment is of the form

$$
\sigma_{L}-\sigma_{R} \propto\left(\bar{\psi}_{L} \gamma^{\nu} \psi_{L}\right)^{2}-\left(\bar{\psi}_{R} \gamma^{\nu} \psi_{R}\right)^{2} \Longrightarrow \delta\left(A_{P V}\right) \propto \frac{e^{2}\left(Q_{L}^{2}-Q_{R}^{2}\right)}{M_{Z^{\prime}}^{2}}
$$

where $\psi_{L, R}$ and $\bar{\psi}_{L, R}$ are the left- and right-handed electron chiral spinors, $\gamma^{\nu}$ are the Dirac matrices, and $e Q_{L}$ and $e Q_{R}$ are chiral couplings of the electron to the $Z^{\prime}$ boson. The sensitivity of the E158 experiment to new bosons can be defined as the minimum $Z^{\prime}$ mass required to produce a result two standard deviations away from the Standard Model prediction. For specific models, this definition leads to a sensitivity ranging from 600 to $900 \mathrm{GeV}$ [10], comparable to the sensitivity expected for results from Run II at the Tevatron [24].

Other new physics effects can arise from new contact interactions, such as an interaction mediated by a doubly charged Higgs boson $\Delta^{--}[26]$. The Lagrangian for the contact interaction is given by [19]

$$
\mathcal{L}_{e e}=\frac{4 \pi}{2 \Lambda_{e} e^{2}}\left[\eta_{L L}\left(\bar{\psi}_{L} \gamma^{\nu} \psi_{L}\right)^{2}+\eta_{R R}\left(\bar{\psi}_{R} \gamma^{\nu} \psi_{R}\right)^{2}+2 \eta_{L R}\left(\bar{\psi}_{R} \gamma^{\nu} \psi_{R}\right)\left(\bar{\psi}_{L} \gamma^{\nu} \psi_{L}\right)\right]
$$


where $\Lambda_{e e}$ is the energy scale at which the electron compositeness becomes important and $\eta_{i f}$ are free parameters, such that $\left|\eta_{i f}\right| \leq 1$. If there are parity-violating terms in the Lagrangian, that is, $\eta_{L L}$ or $\eta_{R R}$ are \pm 1 , then the E158 measurement can set a $2 \sigma$ limit on $\Lambda_{e e}$ at $\sim 10 \mathrm{TeV}[10]$. This result is better than what is obtained from current $e^{+} e^{-}$collider experiments, whose limits on $\Lambda_{e e}$ are in the range of 1 to $4 \mathrm{TeV}$ [54].

New physics at heavy mass scales can modify the effective coupling for low energy electroweak processes through higher-order contributions to the $W$ and $Z$ propagators [48]. The modifications are known as oblique corrections, and can be parametrized by a set of six parameters $(S, T, U, V, W$ and $X)[40]$ [25]. In order to constrain all parameters independently, electroweak measurements have to be performed at energies away from the $Z^{0}$ pole. The E158 parity-violation measurement at low energy is particularly sensitive to the $X$ parameter, which is sensitive to the running of $\sin ^{2} \theta_{W}$. If $X$ turns out to be nonzero, it is a good indication that the new physics scale is close to the electroweak scale and that the new physics does not couple strongly to the $Z^{0}$ boson. The world average so far for $X$ is $0.13 \pm 0.51$ [30], which is consistent with 0, but has a large uncertainty. SLAC Experiment E158 is sensitive to $\delta(X)$ at the level of 0.1 .

The sensitivity to a variety of new physics scenarios, combined with the necessity for measuring the running of the weak mixing angle as a test of the Standard Model, provide a strong motivation for the E158 experiment. Furthermore, the E158 results place important new physics constraints relevant to the Tevatron Run II, which has just started and before the LHC experiments turn on.

\subsection{Overview of the Experiment}

To achieve the goal of measuring the weak mixing angle to a precision of $\delta\left(\sin ^{2} \theta_{w}\right) \sim$ 0.001 the parity-violating asymmetry $A_{P V}$ has to be measured to a precision of $10^{-8}$. This task requires a highly polarized electron beam, a large luminosity, and stringent control over beam systematic effects. The E158 experiment used the SLAC polarized electron source to produce beams with $\sim 80 \%$ polarization containing $3.5-4 \times 10^{11}$ 
electrons per pulse. The polarized electrons scattered off unpolarized electrons in a liquid hydrogen target. The number of scattered Møller electrons was $2-4.5 \times 10^{7}$ particles per pulse. The scattered flux was detected by a total absorption shower calorimeter (Figure 1.7). For this technique to work, the Møller electrons had to be focused on the detector region and separated as much as possible from the backgrounds.

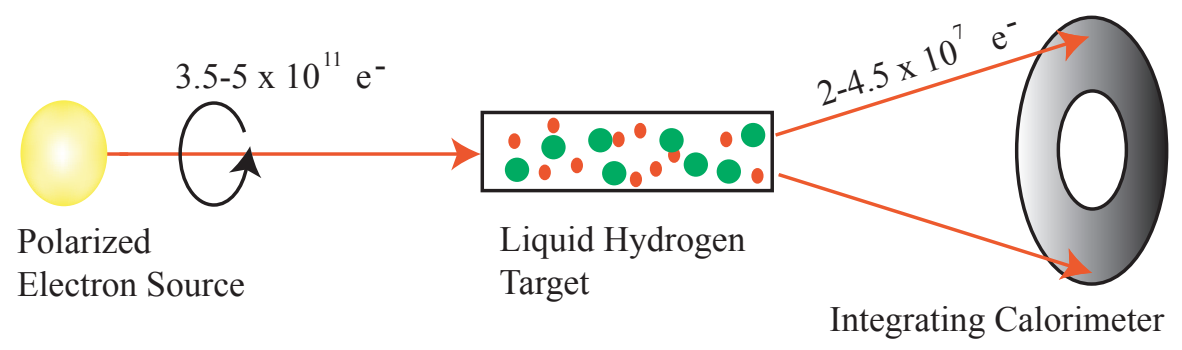

Figure 1.7: Schematic of the E158 experimental design.

The focusing of the signal and defocusing of the backgrounds (consisting mostly of Mott scatters) was accomplished with the help of a magnetic spectrometer. In designing the spectrometer acceptance, the figure of merit (f.o.m.) was taken into account. The f.o.m. parameter quantifies the variation of the statistical uncertainty of the experiment with respect to the scattering angle in the center of mass frame. For the E158 experiment, the f.o.m. $\sim\left(A_{P V}\right)^{2} \times \frac{d \sigma}{d \Omega}$ and its variation with $\cos \Theta_{c m}$ is given in Figure 1.8. The f.o.m varies slowly with $\left|\cos \Theta_{c m}\right|$ with the maximum located at $\cos \Theta_{c m}=0$, which corresponds to electrons having an energy of $24 \mathrm{GeV}$. In order to avoid double-counting the Møller interactions, which is the case when both the incoming electron and the target electron from which it scatters hit the detector, the spectrometer had to accept Møller electrons either above or below $24 \mathrm{GeV}$. Since the lower momentum Møller electrons scatter at a wider angle, the spectrometer was designed to accept Møller electrons in the range of $-0.5<\cos \Theta_{c m}<0$ around the azimuth, corresponding to a momentum range of $12-24 \mathrm{GeV} / \mathrm{c}$ and a total cross section of $\sim 14 \mu$ Barns.

The E158 experiment was approved in September 1997. Construction began in 


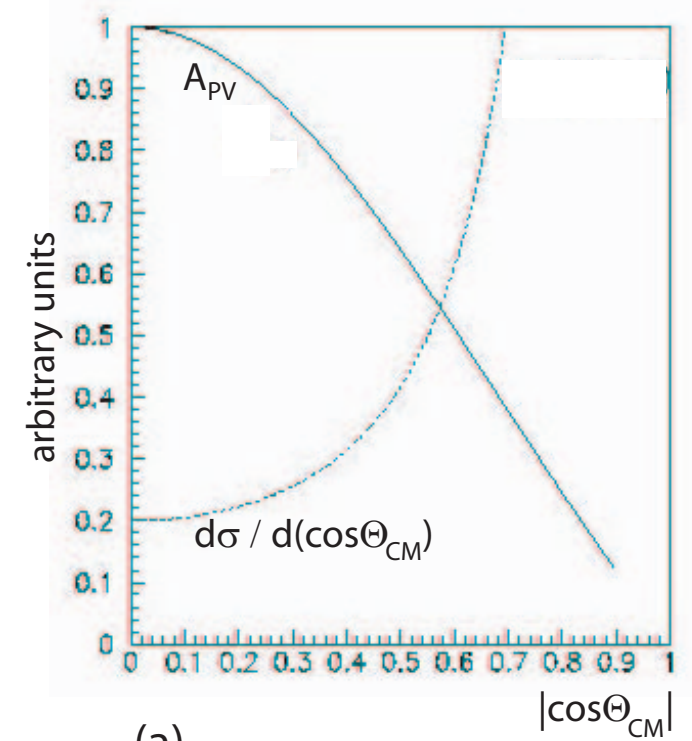

(a)

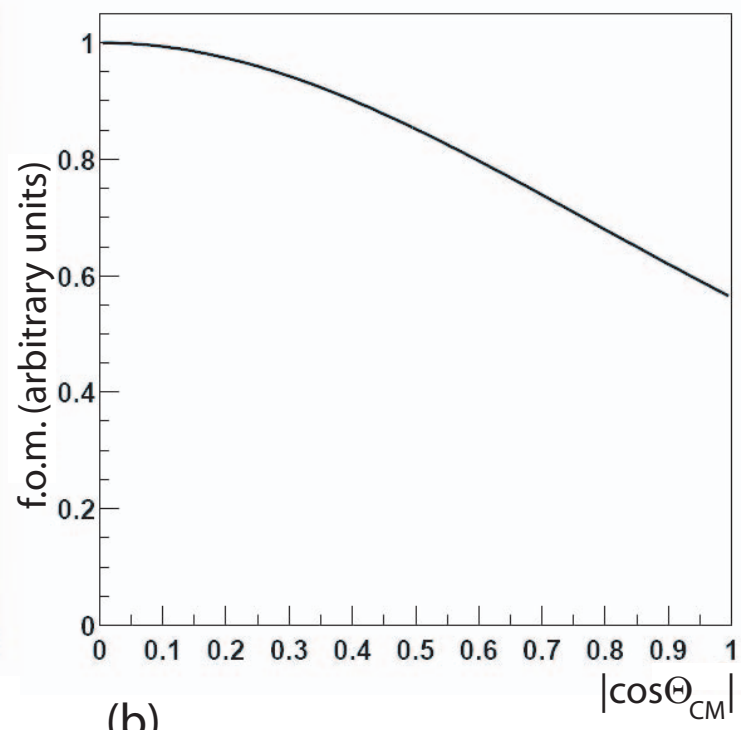

(b)

Figure 1.8: (a) The parity-violating asymmetry and the differential scattering cross section as a function of $\left|\cos \Theta_{c m}\right|$, where $\Theta_{c m}$ is the scattering angle in the center of mass frame. (b) The figure of merit (f.o.m) as a function of $\left|\cos \Theta_{c m}\right|$.

2000. Initial commissioning took place in 2001 followed by three data collection periods in spring 2002, fall 2002 and summer 2003. The data collection periods are known as Run I, Run II and Run III, respectively. In this document the combined results from Run I and Run II are presented. The following chapters contain a detailed description of the E158 experiment consisting of the polarized source (Chapter 2), beam monitoring (Chapter 3), the target and the spectrometer (Chapter 4), and the detectors (Chapter 5). The analysis and results are given in Chapter 6. Chapter 7 presents the value of the weak mixing angle and the conclusions. 


\section{Chapter 2}

\section{Polarized Electron Source and Feedbacks}

SLAC Experiment E158 used a polarized electron source, in which polarized electrons are produced via photoemission from a strained GaAs cathode. The source requirements were the production of $\sim 5 \cdot 10^{11}$ electrons per pulse, polarization of $\sim 80 \%$, and stringent control of helicity-correlated beam asymmetries. An important device used in minimizing helicity-correlated beam asymmetries was an active feedback system. This chapter gives a description of the main source components, and, in particular, the feedback system.

\subsection{The source}

An overview of the E158 source configuration is shown in Figure 2.1. The source consisted of four main parts, all housed in a temperature controlled environment upstream of the accelerator. The assembly began with the laser bench containing the laser and the pulse-shaping optics, followed by a diagnostics bench. The beam polarization was established at the helicity control bench, after which the beam was taken via the optical transport system to the cathode diagnostics bench, where it was finally sent to the cathode. Upon exiting the cathode, the emitted polarized electron beam was bent by $38^{\circ}$ as it entered the accelerator. 


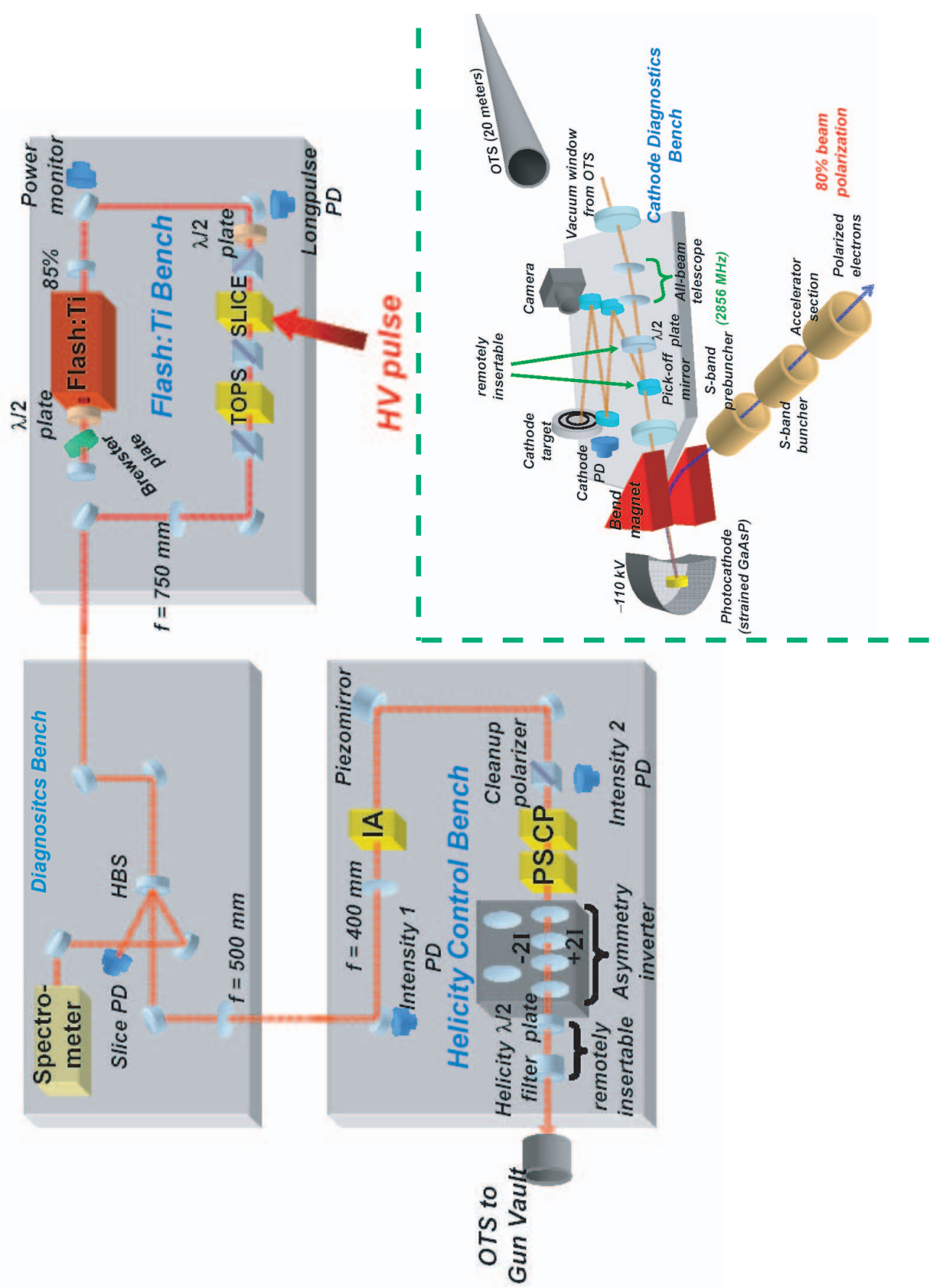

Figure 2.1: Overview of E158 polarized source. 


\subsubsection{The Laser System}

The flashlamp-pumped Ti:Sapphire laser [32] was designed at SLAC and custom built by Big Sky Laser Technologies. Pulse properties of the laser are given in Table 2.1. The laser consisted of a cylindrical Ti:Sapphire crystal centered between two flashlamps, each coupled with an elliptically shaped, rhodium coated reflector. The rhodium was chosen for its mechanical and chemical durability, which minimized laser maintenance work. The operating wavelength and bandwidth of the laser were selected such that the polarization of the photoemitted electrons from the cathode was at its maximum. This corresponded to a wavelength of $805 \mathrm{~nm}$ with a bandwidth of $0.7 \mathrm{~nm}$, narrow enough to ensure that all electrons were emitted at the maximum possible polarization. To maximize the laser output power and minimize pulse-topulse jitter, a one meter cavity was created by using an 85\%-reflective planar output coupler on one end with a 99.9\%-reflective concave mirror on the other end (Figure 2.1). A Brewster plate was added to the cavity in order to optimize transmission for the desired output wavelength and bandwidth.

\begin{tabular}{|l|c|}
\hline s Wavelength & $805 \mathrm{~nm}$ (out of $750-850 \mathrm{~nm}$ range) \\
\hline Bandwidth & $0.7 \mathrm{~nm} \mathrm{FWHM}$ \\
\hline Repetition rate & $120 \mathrm{~Hz}$ \\
\hline Pulse length & $270 \mathrm{~ns}$ (out of $50-370 \mathrm{~ns}$ range) \\
\hline Pulse energy & $60 \mu \mathrm{J}(600 \mu \mathrm{J}$ maximum) \\
\hline Circular polarization & $99.8 \%$ \\
\hline Energy jitter & $0.5 \% \mathrm{rms}$ \\
\hline Position jitter at photocathode & $<70 \mu \mathrm{m} \mathrm{rms}$ \\
\hline
\end{tabular}

Table 2.1: Laser pulse properties.

The laser cavity was followed by pulse-shaping optics, consisting of two Pockels cells sandwiched in between three polarizers. A Pockels cell is a crystal, which becomes birefringent when a high voltage is applied to it. The first Pockels cell in the series was the SLICE Pockels cell, used to determine the laser pulse length and intensity. It was driven by a high voltage pulser with the gate set such that it cut a $270 \mathrm{~ns}$ slice out of $\sim 15 \mu$ s laser pulse (Figure 2.2). The intensity of the laser pulse was controlled by the amplitude of the high voltage, which was part of our feedback system. 
As it will be shown in Section 2.2.2, this feedback not only regulated the electron beam intensity, but it compensated for the decrease in the Quantum Efficiency of the cathode and degradation of the laser flashlamps.

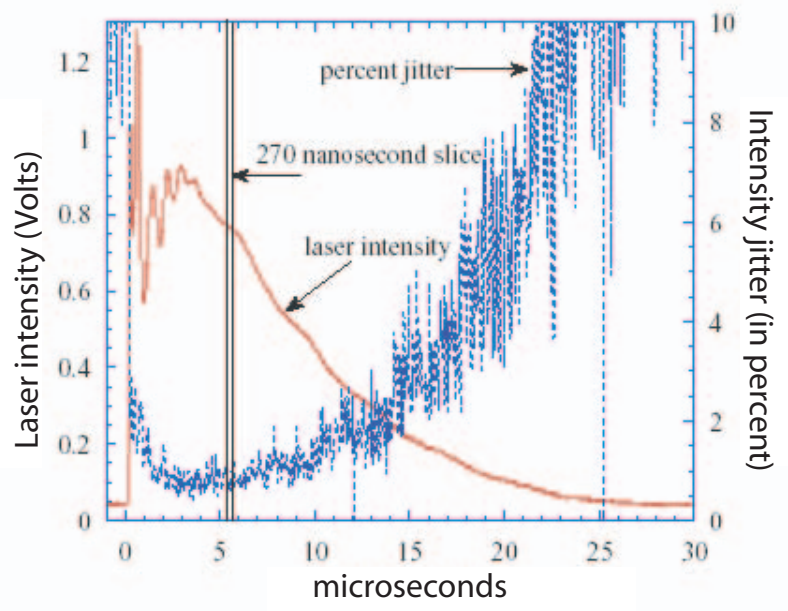

Figure 2.2: Laser intensity and the percent jitter as a function of time within the pulse. The SLICE Pockels cell selects a 270 ns section at the region with the lowest jitter.

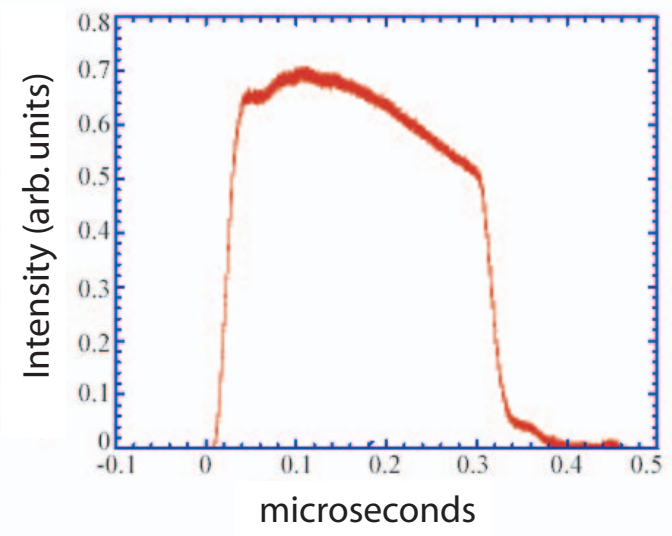

Figure 2.3: Temporal profile of the laser pulse.

The second Pockels cell was a Top-hat Pulse Shaper (TOPS) Pockels cell. It established the temporal profile of the laser pulse, chosen to compensate for a phenomenon known as "beam loading." As electrons cross through an accelerating cavity, they 
absorb power from it, with the result being that the electrons arriving later in the pulse have less power available to them and therefore less energy. If we had used a flat temporal profile, "beam loading" would have induced an energy spread along the pulse length. Instead, the pulse shape shown in Figure 2.3 was used.

\subsubsection{Diagnostics Bench}

The characteristics of the laser beam were monitored in the Diagnostics Bench (Figure 2.4). A holographic beam sampler (HBS) divided the laser beam into three branches with the main one traveling through to the Helicity Control Bench. The other two branches consisted of samples of $1 \%$ of the beam each. One sample was used to monitor the intensity of the pulse with a photocathode (Slice PD), while the other was sent to a spectrometer, where one could measure the laser beam wavelength.

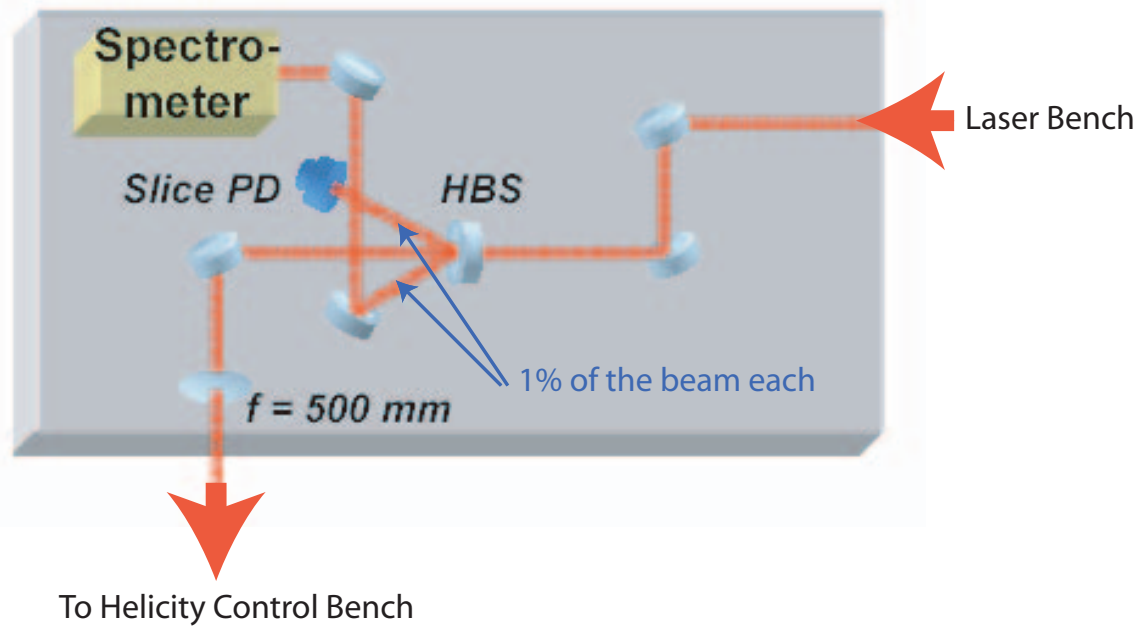

Figure 2.4: Diagnostics Bench. 


\subsubsection{Helicity Control Bench}

At the Helicity Control Bench circularly polarized light was produced on a pulseby-pulse basis. The helicity was selected for each pulse and will be discussed in section 2.2.1. The purpose of the Helicity Control Bench was not only to generate highly polarized beam, but to reduce beam helicity-correlated asymmetries. The polarization of the beam was determined by three Pockels cells, shown in Figure 2.1 as Cleanup Polarizer, Circular Polarization (CP), and Phase Shift (PS). The Cleanup Polarizer served to combine the E158 laser beam with the beam generated for the BaBar experiment ${ }^{1}$, enabling the beams for both experiments to share the remaining source optics. The CP cell acted as a quarter wave plate, whose fast axis was $45^{\circ}$ off the horizontal, and whose retardation sign could be switched on a pulse-by-pulse basis, producing either left- or right-helicity of the light. The definition of left- and right-helicities for E158 is given in Figure 2.5. One could adjust the voltage of the $\mathrm{CP}$ cell (typical operating value was $2.7 \mathrm{kV}$ ) to compensate for residual linear polarization along the horizontal or vertical axis. In addition, the residual linear polarization along the $\pm 45^{\circ}$ axis was minimized with the help of the PS cell. The final circular polarization was $\geq 99.8 \%$ with an unpolarized component $\leq 0.2 \%$.
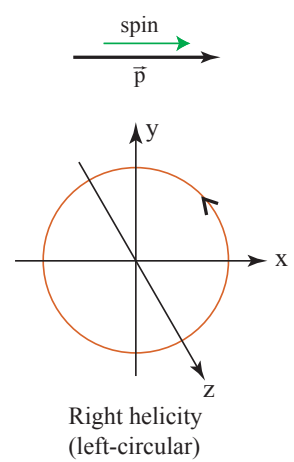
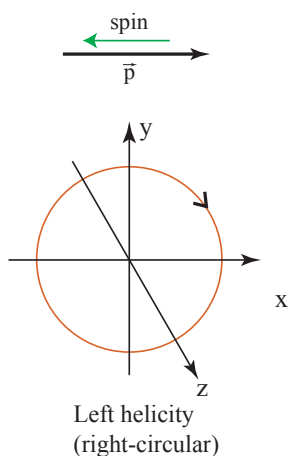

Figure 2.5: Definition of right- and left-helicity for the electron beam (top) and the laser beam polarization (bottom).

An important tool in reducing helicity-correlated systematics was to employ slow helicity reversals. At the source this was accomplished in two ways: by flipping the

\footnotetext{
${ }^{1}$ During Run I, data collection for E158 occurred concurrently with the BaBar experiment.
} 
sign of the beam asymmetry but leaving the physics asymmetry unchanged, and by flipping both the physics and the beam asymmetry. The first was achieved via an Asymmetry Inverter, which consisted of a series of four lenses mounted in parallel, downstream of the polarization optics. These lenses inverted the position and intensity asymmetry of the beam without changing its helicity [32]. The physics and beam asymmetry reversal was achieved by inserting a zeroth-order half-wave plate, which flipped the helicity independently of the Pockels cells. The insertable half-wave plate was located in the Cathode Diagnostics Bench.

\subsubsection{Cathode Diagnostics Bench}

The beam was transported from the Helicity Control Bench through the Optical Transport System (OTS), which was just a 20 m long pipe containing a converging lens and a system of mirrors. Once it entered the Cathode Diagnostics Bench, the beam was directed through two telescope lenses. These lenses served to image the CP cell onto the cathode (Figure 2.1), in order to minimize possible beam asymmetries generated while the beam was steered from the $\mathrm{CP}$ cell to the cathode. Following the telescope lenses was the insertable half-wave plate mentioned in the previous paragraph. An insertable $50 \%$ pick-off mirror was used to send the beam through the auxiliary diagnostics line in order to measure the beam spotsize. The reason for a separate diagnostics line was to reduce the number of optical elements in the transport system, so that a high degree of circular polarization was ultimately maintained. At this point, the polarized beam was ready to hit the cathode.

\subsubsection{The Cathode}

During Experiment E158, a new gradient-doped strained GaAs cathode was used, which could yield up to $2 \cdot 10^{12}$ electrons at a polarization greater than $80 \%$, given our available laser power. High charge and polarization were achieved by applying a strain to a $100 \mathrm{~nm}$ deep surface layer of the cathode, which broke the degeneracy of the $P_{3 / 2}$ energy levels in GaAs and increased the level of polarization. Doping the 
top $10 \mathrm{~nm}$ of that layer with high levels of $\mathrm{Zn}$, forced the GaAs crystal to overcome a charge limit [42]. A schematic of the various GaAs layers is shown in Figure 2.6.

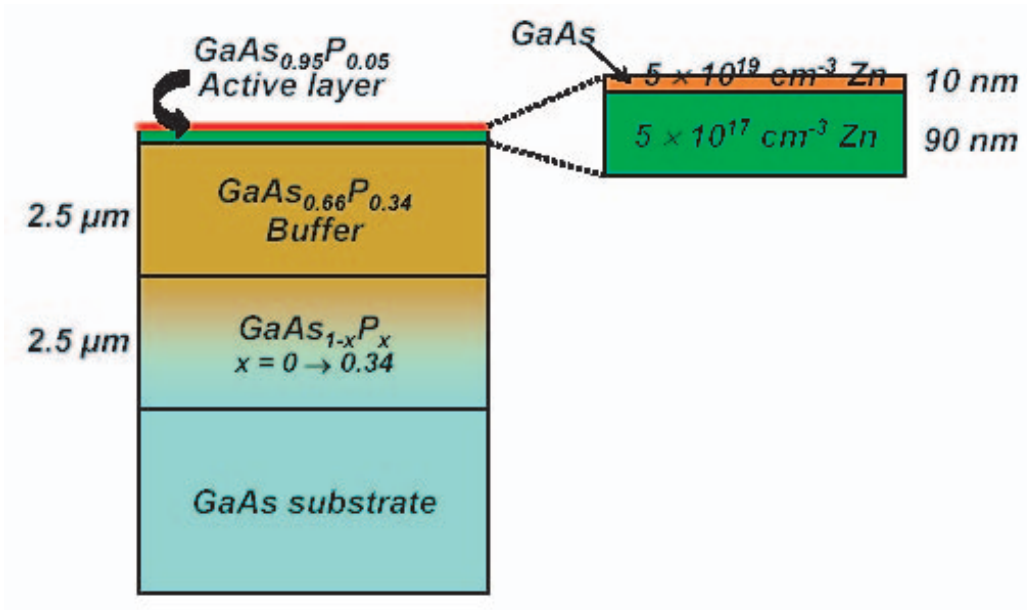

Figure 2.6: Gradient-doped strained GaAs cathode.

The band-gap diagram for GaAs is illustrated in Figure 2.7. Laser light pumped electrons from the valence band into the conduction band, with right-helicity light exciting electrons from the $P_{3 / 2}, m_{J}=-3 / 2$ to the $S_{1 / 2}, m_{J}=-1 / 2$ level and lefthelicity light exciting electrons from the $P_{3 / 2}, m_{J}=+3 / 2$ to the $S_{1 / 2}, m_{J}=+1 / 2$ level. The cathode was operated such that the emitted electrons moved in the opposite direction of the incoming light, so that right- and left-helicity laser light yielded rightand left-helicity electrons, respectively, as defined in Figure 2.5. Once the electrons left the cathode they were ready for acceleration.

\subsection{The Feedback System}

In the E158 experiment, it was important to minimize systematic uncertainties to the level of a few parts per billion. This was partially achieved by periodically flipping the helicity to minimize drifts, as well as by employing active feedbacks. We used slow and fast asymmetry reversals. The slow asymmetry reversals are described in section 2.1.3. The fast asymmetry reversals were accomplished by flipping the beam 


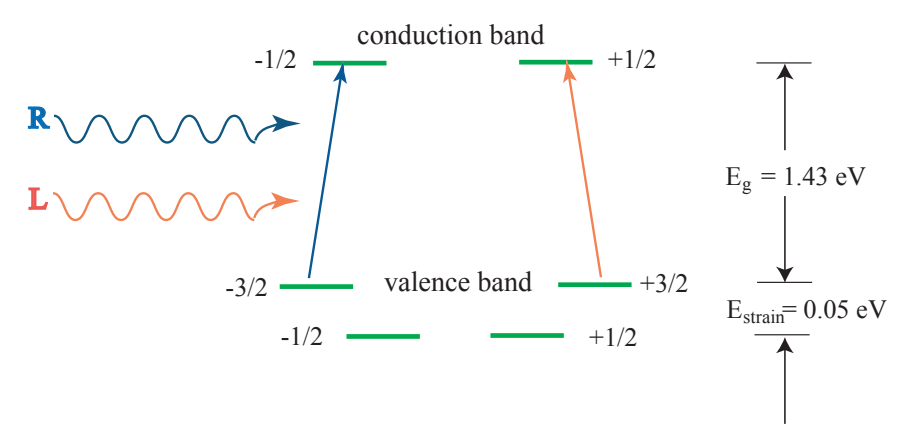

Figure 2.7: Band-gap diagram for GaAs.

helicity on a pulse-by-pulse basis to reduce the effects from energy, intensity and position drifts for each helicity.

\subsubsection{Helicity Sequence}

The beam helicity was governed by the Polarization Monitor (PMON), a SLAC built electronic system, which controlled the source optics and was read out by the E158 data acquisition (DAQ). PMON generated a pseudo-random helicity sequence, using a 33-bit shift register algorithm [31]. Pulses were produced at a rate of $120 \mathrm{~Hz}$, which were grouped into quadruplets at $30 \mathrm{~Hz}$ each. The helicities of the first two members of the quadruplet were chosen randomly by PMON, while the subsequent two were complements of the first two pulses, respectively. For example, a sequence of quadruplets could be LLRR-RLLR-LRRL and so on. In the analysis we calculated the asymmetry for each pulse pair, where a pair was the first and third member of the quadruplet, or the second and fourth. The reason for devising the helicity sequence this way was that pairs came at $60 \mathrm{~Hz}$, which means that members of the same pair were at the same phase with respect to the $60 \mathrm{~Hz} \mathrm{AC}$ noise, inherent in the accelerator environment. Additionally, this sequencing enabled running the experiment in two independent $60 \mathrm{~Hz}$ timeslots, with each pair from the quadruplet corresponding to 
one timeslot. Analyzing the data from each timeslot separately created the benefit of essentially conducting two independent experiments. Apart from determining the helicity sequence, PMON controlled all the helicity-correlated devices at the source, such as setting the voltages for the PS and CP cells, as well recording in the E158 DAQ pulse helicities and their identification numbers.

\subsubsection{Active Feedback Loops and Feedback Algorithm}

Helicity-correlated beam asymmetries were suppressed with the help of three active feedback loops located in the Helicity Control Bench (Figure 2.1). The "IA loop", which was just another Pockels cell, induced intensity asymmetry into the laser beam, which compensated for the measured intensity asymmetry on the electron beam. The "POS loop", which was a piezo mirror, could change the angle of the laser beam through the optics system, which translated into helicity-correlated displacements of the laser beam on the cathode. This system helped correct for measured electron beam position asymmetries. The third loop, "Phase Feedback", fed back on the CP and PS cell voltages, adjusting them in order to keep the correction induced by the "IA loop" small. It provided a second layer of feedback by minimizing the effect of drifts in the polarization state of the laser beam, which created laser beam intensity asymmetries.

The goal of the feedback algorithm was to use the feedback loops such that the beam asymmetry over a large number of runs was minimized. The electron beam asymmetry $A_{\text {beam }}$, measured by the beam monitors described in Chapter 3 , averaged over a mini-run $n$ is given by ${ }^{2}$

$$
A_{\text {beam }}^{n}=A_{\text {fbk }}^{n}+A_{\text {source }}^{n}+A_{\text {stat }}^{n},
$$

where $A_{f b k}^{n}$ is the asymmetry induced by the feedback loops, $A_{\text {source }}^{n}$ is the helicitycorrelated asymmetry caused by the source, and $A_{\text {stat }}^{n}$ is due to statistical fluctuations

\footnotetext{
${ }^{2}$ The number of pairs in each mini-run was determined by the size of the jitter, in order to collect sufficient statistics. We collected 800 pairs for the intensity feedback (IA loop), 10000 pairs for the position feedback (POS loop), and 24000 pairs for the Phase Feedback.
} 
in the electron beam. For each mini-run we set $A_{f b k}^{n}$ according to the following algorithm:

$$
\begin{aligned}
& A_{f b k}^{1}=0 \\
& A_{f b k}^{2}=-g A_{\text {beam }}^{1} \\
& A_{f b k}^{3}=A_{f b k}^{2}-g A_{\text {beam }}^{2} \\
& \cdots \ldots \\
& A_{f b k}^{n}=A_{f b k}^{n-1}-g A_{\text {beam }}^{n-1},
\end{aligned}
$$

where $g$ is the feedback loop gain.

In the ideal situation, $g=1, A_{\text {source }}$ is constant, and the feedback loops have perfect resolution. In this case, when averaged over $N$ mini-runs, the feedback algorithm would yield

$$
\begin{aligned}
<A_{\text {beam }}> & =\frac{1}{N} \sum_{n=1}^{N} A_{\text {beam }}^{n} \\
& =\frac{1}{N}\left(A_{\text {source }}+A_{\text {stat }}^{1}-A_{\text {stat }}^{1}+A_{\text {stat }}^{2}-\ldots-A_{\text {stat }}^{N-1}+A_{\text {stat }}^{N}\right) \\
& =\frac{1}{N}\left(A_{\text {source }}+A_{\text {stat }}^{N}\right) .
\end{aligned}
$$

Instead of $1 / \sqrt{N}$ scaling that one would expect from pure statistical behavior, the active feedbacks cause the beam asymmetry measured over $N$ runs to scale as $1 / N$.

In reality, several factors limited the $1 / N$ scaling. The gain for each loop was not exactly unity and the devices used by the feedback loops had finite resolutions. Most importantly, we ran the feedbacks at the source, where the beam energy was just 1 $\mathrm{GeV}$, while the Møller detector was in the experimental hall downstream of the 2 mile accelerator with the beam at 45-48 GeV. By nulling the asymmetries before the beam was accelerated, the asymmetries at the detector were minimized. During the experiment, no energy feedback was implemented. However, since beam intensity and energy were correlated due to beam loading, the existence of the intensity feedback at the source helped maintain the energy helicity-correlated asymmetries to a reasonable 
level.

During the experiment typical IA loop induced corrections were of the order of \pm 100 ppm, while the average induced correction was 0.69 ppm. The POS loop induced a correction of $-24 \mathrm{~nm}$ in $\mathrm{x}$ and $2 \mathrm{~nm}$ in $\mathrm{y}$ [43]. The plots in Figure 2.8 illustrate how the feedbacks worked during the experiment.

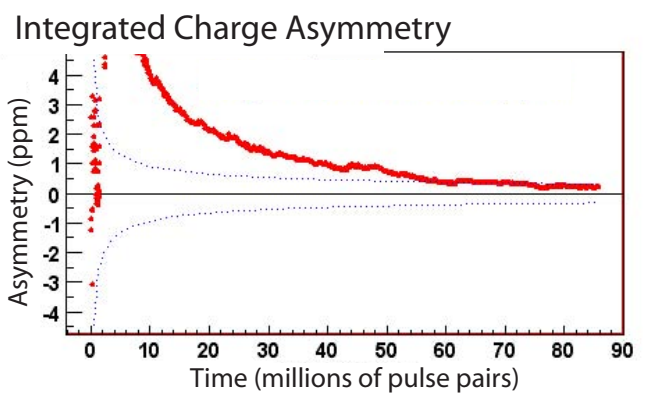

(a)

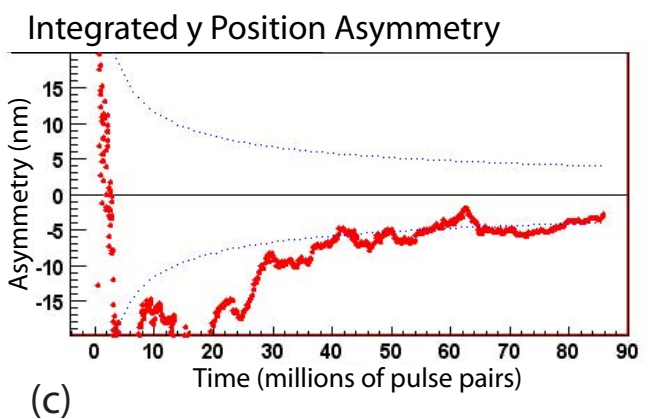

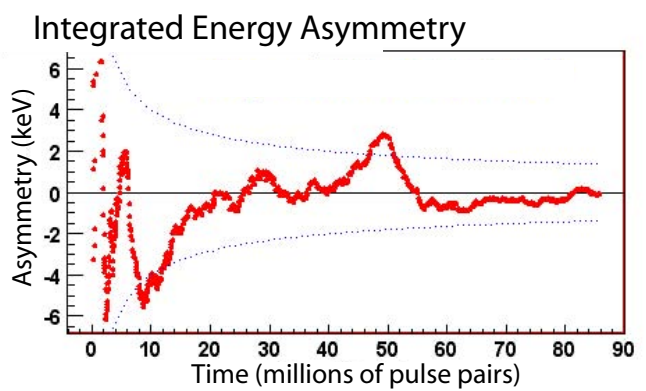

(b)

Figure 2.8: Feedback performance during Run I. The dotted line corresponds to $\frac{1}{\sqrt{N}}$ statistical scaling. (a) Plot of the integrated charge asymmetry in parts per million (ppm). (b) Integrated energy asymmetry in keV. (c) Integrated y position asymmetry in $\mathrm{nm}$. 


\section{Chapter 3}

\section{Precision Beam Monitoring}

From the source to the detectors the beam has a long journey, starting with the linear accelerator, continuing with the A-Line and ending at the experimental hall known as End Station A (ESA), which housed the target, the spectrometer and the detectors (Figure 3.1). The accelerator at SLAC contains 30 sectors of 8 klystrons each. A klystron, invented in 1936 by Hansen and Varian brothers, consists of a copper cavity driven by 65 megawatt, $2856 \mathrm{MHz} \mathrm{RF}$ power [45]. Each structure can accelerate electrons up to $200 \mathrm{MeV}$. After leaving the accelerator the beam is bent by $24.5^{\circ}$ in order to enter ESA. The bend is achieved in a separate beamline called the "A-Line" where the beam is steered using 12 quadrupole and 12 dipole magnets. The effect of the bend provides a g-2 spin precession of the electrons, which, during the E158 experiment, was exploited as a way of performing a slow asymmetry reversal. First, we selected to run at an energy for which the beam spin direction in ESA was purely longitudinal. Second, we ran at two such energies, 45 and $48.3 \mathrm{GeV}$, because the energy change corresponded to a reversal of the electron spin direction.

Each beam pulse consisted of a $270 \mathrm{~ns}$ long electron bunch train, where each bunch arrived at a $2856 \mathrm{MHz}$ rate. One $\mathrm{Hz}$ of pedestal pulses, containing no beam, were used for pedestal subtractions. It was paramount for the E158 experiment to measure and monitor beam parameters very precisely on a pulse by pulse basis, in order to minimize their contribution to the physics asymmetry width. We had monitors for beam charge, position, energy, and spot size, as well as a synchrotron light monitor used to detect beam energy fluctuations. These monitors were located throughout 


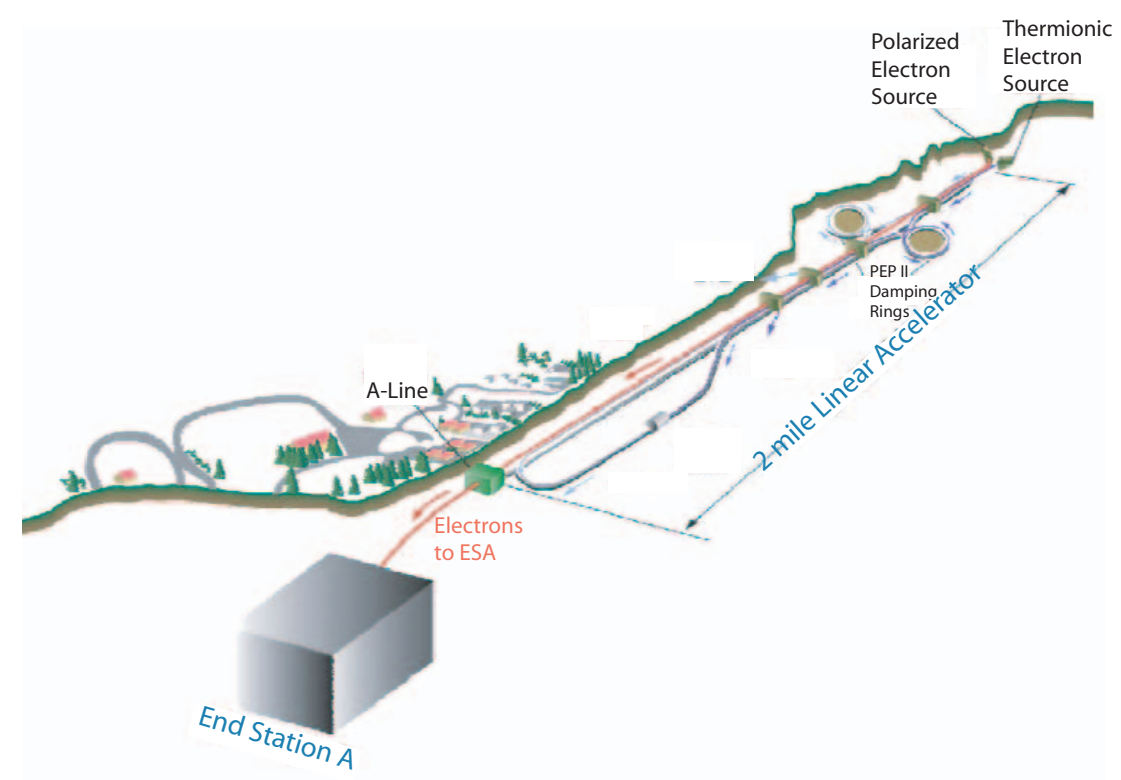

Figure 3.1: Overview of the SLAC 2 mile accelerator, A-Line and End Station A.

the accelerator, A-Line and ESA and are described in the following sections.

\subsection{Charge Monitors}

The beam charge was monitored for every pulse using toroids consisting of a doughnutlike iron core wrapped with copper wire [46]. The wire was connected to a resistor and a capacitor, thus creating an RLC circuit. When crossed by an electron beam, a pulse would be induced in the coils, producing a ringing signal in the RLC circuit. The signal was amplified by a set of amplifiers, whose gains could be set remotely. In order to reduce pickup noise, the amplifiers were located near the toroids, only a few feet away from the beam. To prevent radiation damage, they were enclosed in lead brick huts. The rest of the beam monitoring electronics was located in the counting house - a radiation shielded room adjacent to ESA, which contained most of the DAQ electronics.

In the counting house, the signal was rectified to enable integration by a 16-bit custom-designed Analog to Digital Converter (ADC). The larger the height of the ADC signal, the better the resolution, since ADCs had fixed pedestal noise (1 count 
for toroid ADCs). As a result, the Q of the RLC toroid circuit was set to be as high as possible with the integration lasting for longer than $1 \mathrm{~ms}$. One drawback was that, with such a large Q, approximately $1 \%$ of the charge per pulse would "leak" into the next pulse. This difficulty was solved by damping the pulse after $3 \mathrm{~ms}$, which was well after the integration gate for the pulse had closed, but prior to the integration gate for the subsequent pulse. Toroids were calibrated by injecting a calibration signal of known charge and comparing the calibration signal to the ADC output. Figure 3.2a shows a photograph of a pair of toroids. Figure $3.2 \mathrm{~b}$ presents a schematic of the toroid stand. Each toroid was surrounded by an aluminum shield, and the beam pipe for that section was replaced with ceramic, both intended to prevent interferences with the toroid pickup.

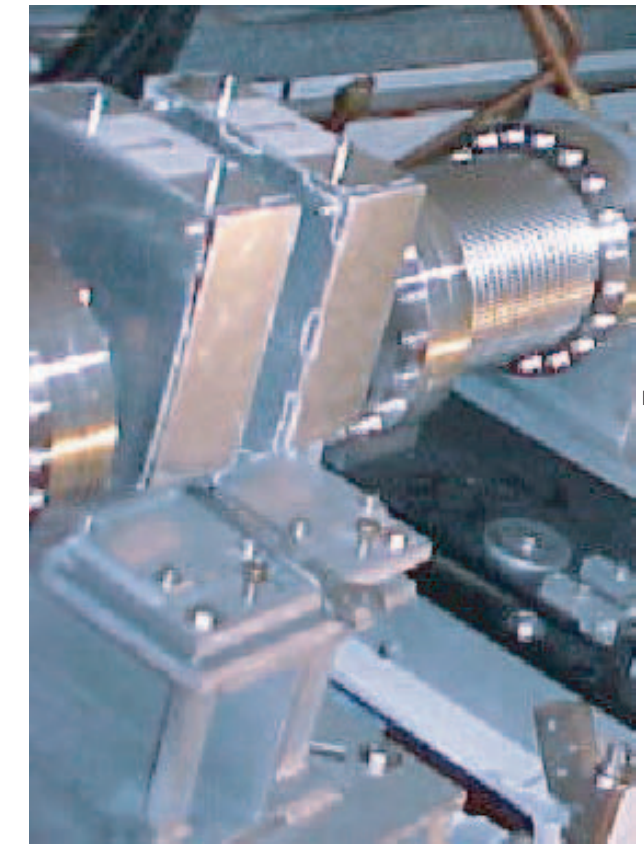

(a)

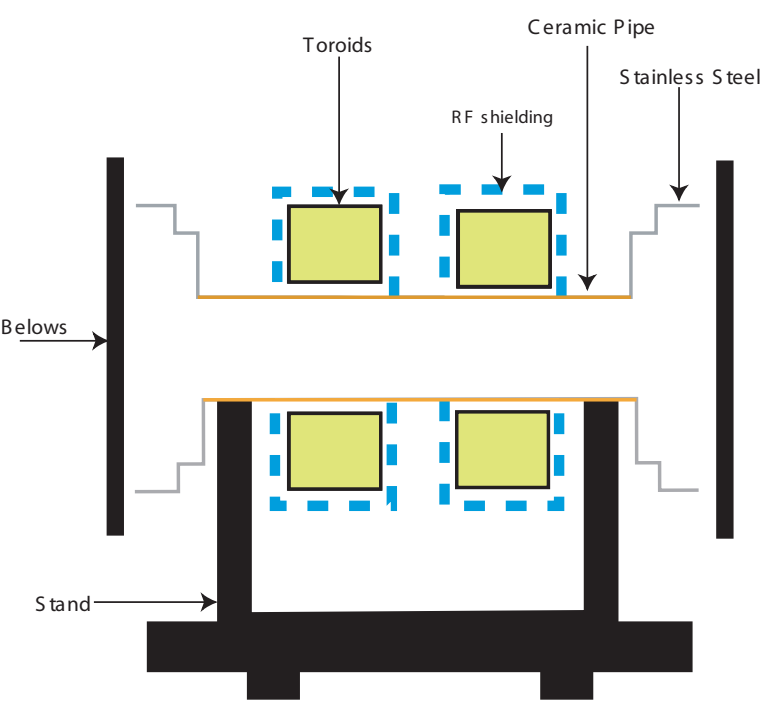

(b)

Figure 3.2: Mechanical design of E158 toroids.

Three pairs of toroids were used to measure charge. One pair was installed in the $\mathrm{ASSET}^{1}$ region, while the other two pairs were installed at the entrance of ESA

\footnotetext{
${ }^{1}$ ASSET stands for Accelerator Setup Structure for Experimental Testing. ASSET is a $2 \mathrm{~m}$ long
} 
(Figure 3.12.) The location selection is explained in Section 3.2.4. Such a pairing of toroids, not only offered redundancy, but it enabled a measurement of higher resolution than by using a single toroid. To determine the resolution, the charge asymmetry per pulse pair measured by one toroid $\left(\frac{L-R}{L+R}\right)$ was subtracted from the asymmetry measured by the adjacent one. This served to remove beam jitter, which was much larger than the toroid resolution. Typical resolution for ESA toroids during Run I and Run II was 40-60 ppm (Figure 3.3.)

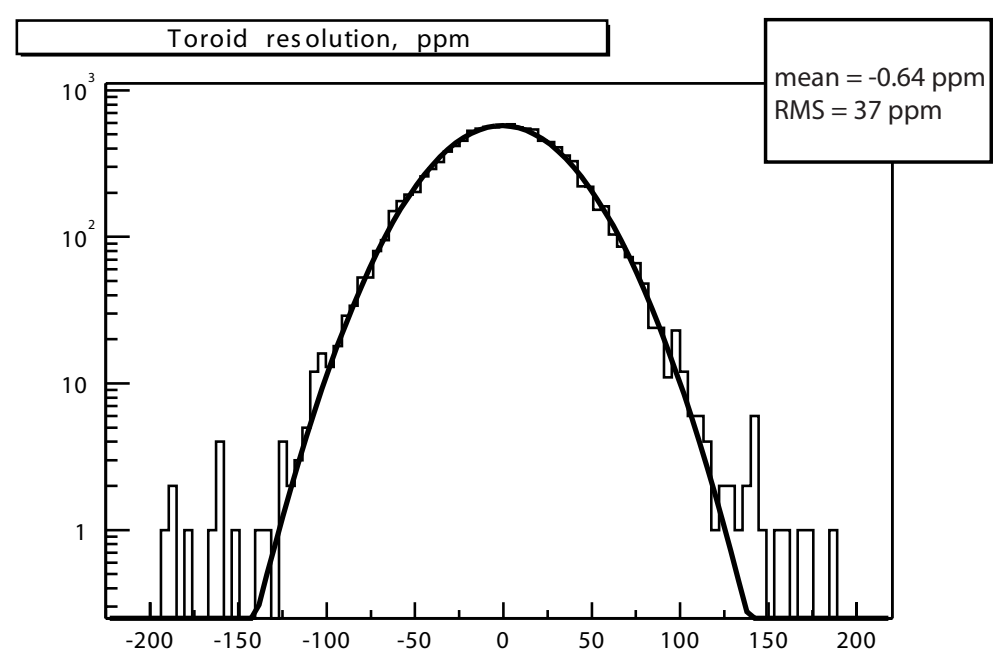

Figure 3.3: Toroid resolution for one run (given by the root mean square), using two of the ESA toroids.

\subsection{Beam Position Monitors}

Beam position, angle and energy were measured using beam position monitors (BPMs) consisting of resonant copper cavities. For Experiment E158 the BPM resolution was improved by a factor of 10 , compared to the past.

region of the accelerator beam line located approximately $250 \mathrm{~m}$ downstream from the source, where the beam energy has reached only $1.2 \mathrm{GeV}$. 


\subsubsection{Theoretical Description of Resonant Cavities}

When charged particles pass through a resonant cavity they excite a superposition of transverse electromagnetic (TEM) modes depending on the cavity geometry, which can be picked up by an antenna inside. The cavity acts as a damped harmonic oscillator, driven by the beam, where the beam multipole moments couple to the cavity modes $\lambda$, each characterized by a resonant frequency $w_{\lambda}$. The E158 BPMs used a resonant cavity with two beam ports, coupled to a waveguide. The pickup voltage $V_{c}$ and the forward and reverse voltages $V_{F}$ and $V_{R}$, shown in Figure 3.4, are given by [67].

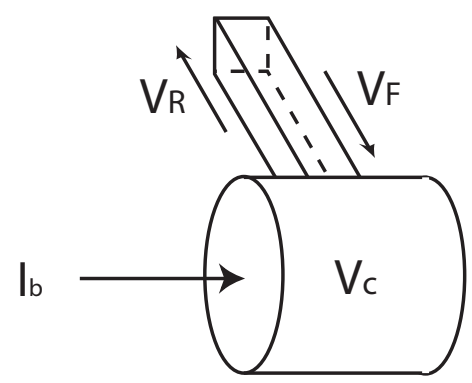

Figure 3.4: Dynamic variables of a cavity coupled to a waveguide and the beam.

$$
\left(\frac{d^{2}}{d t^{2}}+\frac{\omega_{\lambda}}{Q_{L \lambda}} \frac{d}{d t}+\omega_{\lambda}^{2}\right) V_{c \lambda}=2 \frac{\omega_{\lambda}}{Q_{e \lambda}} \frac{d V_{F \lambda}}{d t}-2 K_{\lambda}\left(\mathbf{r}_{\perp b}\right) \frac{d I_{b}}{d t}
$$

Here $V_{c \lambda}=V_{F \lambda}+V_{R \lambda}, I_{b}$ is the beam current waveform, $Q_{e \lambda}$ is the external $Q$, which characterizes the coupling strength between the cavity and the waveguide, $Q_{L \lambda}$ is the port-loaded Q defined as

$$
\frac{1}{Q_{L \lambda}}=\frac{1}{Q_{w \lambda}}+\frac{1}{Q_{e \lambda}},
$$

where $Q_{w \lambda}$ quantifies the wall losses. $K_{\lambda}$ is a generalized loss factor, characterizing the coupling of the beam offset by $\mathbf{r}_{\perp b}$ to the particular cavity mode $\lambda$ and is strongly dependent on the cavity geometry and the beam position. This becomes evident if 
Eq. 3.1 is rewritten as

$$
\left(\frac{d^{2}}{d t^{2}}+\frac{\omega_{\lambda}}{Q_{L \lambda}} \frac{d}{d t}+\omega_{\lambda}^{2}\right) V_{c \lambda} \approx 2 \frac{\omega_{\lambda}}{Q_{e \lambda}} \frac{d V_{F \lambda}}{d t}-2 k_{\lambda} \frac{d}{d t} X_{\lambda}\left(\mathbf{r}_{\perp b}\right) I_{b}(t)
$$

where $k_{\lambda}=K_{\lambda}\left(\mathbf{r}_{\perp 0}\right)$ and $X$ is the beam coordinate function.

\subsubsection{Excitation by Gaussian Bunch}

In the simple case of a tri-Gaussian beam ${ }^{2}$ with charge $Q_{b}$, bunch size $\sigma_{x}$ and $\sigma_{y}$, bunch length $c \sigma_{t}$, with bunch position $\left(x_{b}, y_{b}\right)$ and bunch arrival time $t_{b}$, the current density $J_{b}$ and current waveform $I_{b}$ are given by

$$
\begin{gathered}
J_{b}(x, y, t)=\frac{I_{b}(t)}{2 \pi \sigma_{x} \sigma y} \exp \left[-\frac{\left(x-x_{b}\right)^{2}}{2 \sigma_{x}^{2}}-\frac{\left(y-y_{b}\right)^{2}}{2 \sigma_{y}^{2}}\right] \\
I_{b}(t)=\frac{Q_{b}}{2 \pi^{1 / 2} \sigma_{t}} \exp \left[-\frac{\left(t-t_{b}\right)^{2}}{2 \sigma_{t}^{2}}\right] .
\end{gathered}
$$

Solving Eq. 3.3 for the case with $V_{F}=0$, the ideal case of a perfectly matched output load, one finds

$$
V_{c \lambda}=\mathcal{R} e^{j \Omega_{\lambda}\left(t-t_{b}\right)} \tilde{V}_{c \lambda}(t)
$$

Here $\Omega_{\lambda}^{2}=\omega_{\lambda}^{2}-\frac{1}{4} \nu_{\lambda}^{2}$ and $\nu_{\lambda}=\frac{\omega_{\lambda}}{Q_{L \lambda}}$. The voltage phasor $\tilde{V}_{c \lambda}$ is given by

$$
\tilde{V}_{c \lambda}=-\hat{V}_{c \lambda} H\left(t-t_{b}\right) \exp \left[\frac{1}{2} \nu_{\lambda}\left(t-t_{b}\right)\right]
$$

and the amplitude $\hat{V}_{c \lambda}$ is

$$
\hat{V}_{c \lambda}=-2 k_{\lambda} Q_{b}\left\langle X_{\lambda}\right\rangle \exp \left(-\frac{1}{2} \omega_{\lambda}^{2} \sigma_{t}^{2}\right)
$$

So the cavity pickup voltage $V_{c \lambda}$ has a step-rise at the beam arrival time $t_{b}$ and then it rings with frequency $\Omega_{\lambda}$, while its amplitude decays exponentially. The voltage $V_{c \lambda}$ depends linearly on $\left\langle X_{\lambda}\right\rangle$. For a predominantly monopole mode, such as the case

\footnotetext{
${ }^{2}$ The current density and waveform of each electron bunch has Gaussian distribution.
} 
of a cylindrical cavity, $\left\langle X_{\lambda}\right\rangle$ is to first order independent of beam position:

$$
\langle X\rangle \approx 1+B_{2}^{*}\left(x_{b}^{2}-y_{b}^{2}+\sigma_{x}^{2}-\sigma_{y}^{2}\right)
$$

For a predominantly x-dipole mode (similarly for $\mathrm{y}$ ), such as the case of a rectangular cavity which is symmetric for an $\mathrm{x}$ inversion, $\left\langle X_{\lambda}\right\rangle$ is to first order linearly dependent on beam position:

$$
\langle X\rangle \approx x_{b}+x_{b} B_{3}^{*}\left(x_{b}^{2}-3 y_{b}^{2}+3 \sigma_{x}^{2}-3 \sigma_{y}^{2}\right)
$$

Parameters $B_{2}^{*}$ and $B_{3}^{*}$ characterize the quadrupole and sextupole second-order contributions, respectively.

\subsubsection{Excitation by a Bunch Train}

The E158 bunch train was composed of tri-Gaussian bunches. If one assumes a uniformly bunched beam with small beam size and offset, the voltage induced after the passage of the $\mathrm{n}^{\text {th }}$ bunch can be expressed in terms of the arrival time and amplitude of the first bunch $(n=0)$ :

$$
V_{c \lambda}=\mathcal{R} \exp \left[\Gamma_{\lambda}\left(t-t_{b 0}\right)\right] \hat{V}_{c \lambda 0} \frac{\exp \left(\Gamma_{\lambda} \tau n\right)-1}{\exp \left(\Gamma_{\lambda} \tau\right)-1}
$$

where $\Gamma_{\lambda}=\frac{1}{2} \nu_{\lambda}-j \Omega_{\lambda}, t_{b 0}$ is the arrival time of the first bunch, and the bunch spacing is given by $\tau=\frac{2 \pi}{\Omega_{0}}$ with $\Omega_{0}$ being the accelerator frequency.

If the cavity is tuned to resonance and the beam is perfectly bunched then $\Gamma_{\lambda} \tau \ll$ 1 , and

$$
V_{c \lambda} \approx \mathcal{R} \exp \left[j \Omega_{\lambda}\left(t-t_{b 0}\right)\right] \hat{V}_{e f f, \lambda}
$$

with

$$
\hat{V}_{e f f, \lambda}=\frac{Q_{L \lambda}}{\pi} \hat{V}_{c \lambda 0}=-2 k_{\lambda} Q_{b e f f, \lambda}\left\langle X_{\lambda}\right\rangle \exp \left(-\frac{1}{2} \omega_{\lambda}^{2} \sigma_{t}^{2}\right)
$$

where $Q_{b e f f, \lambda}=Q_{L \lambda} Q_{b} / \pi$. The resonant enhancement is given by $Q_{L \lambda}$ (the portloaded Q) [67]. 


\subsubsection{Detuning from Resonance}

In the case of detuning, the cavity resonant frequency $\Omega_{\lambda}$ is different from the bunch train frequency $\Omega_{0}$. To account for this effect one introduces the tuning angle $\psi_{\lambda}$ defined as

$$
\tan \psi_{\lambda}=Q_{L \lambda}\left(\frac{\Omega_{\lambda}}{\Omega_{0}}-\frac{\Omega_{0}}{\Omega_{\lambda}}\right) \approx 2 Q_{L \lambda}\left(\frac{\Omega_{\lambda}-\Omega_{0}}{\Omega_{0}}\right)
$$

in terms of which Eq. 3.12 can be rewritten as

$$
V_{c \lambda} \approx \mathcal{R} \exp \left[j \Omega_{\lambda}\left(t-t_{b 0}\right)\right] \frac{Q_{L}}{\pi} \hat{V}_{0} \cos \psi e^{j \psi}
$$

One finds that detuning modifies the phase to first order and the amplitude to second order in $\psi$. Since it is the amplitude that contains the information regarding beam position, detuning has a second-order effect on the position measurement.

\subsubsection{Mechanical Design}

The BPMs used by Experiment E158 are composed of three resonant cavities, one cylindrical and two rectangular ones (Figure 3.5). The cylindrical cavity, known as

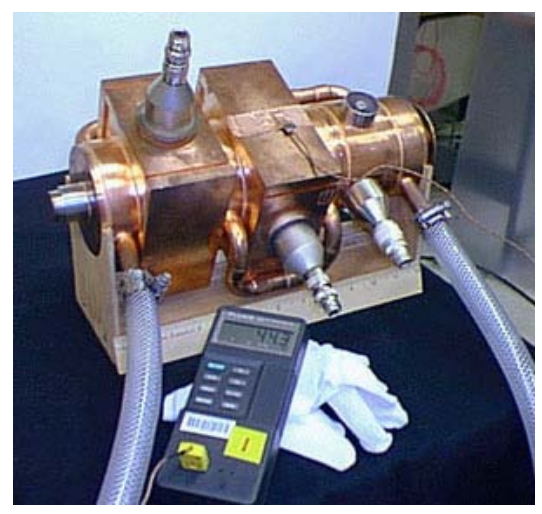

Figure 3.5: E158 BPM.

the $\phi$-cavity, was operated in the monopole mode $T M_{01}$. The cavity is sensitive to beam charge, not position and can be used as a charge monitor. Figure 3.6 shows 
the dimensions of the $\phi$-cavity. Depending on where they were positioned along the beam line, the diameter of the beam aperture in the $\phi$-cavities was 0.8 ", 2 " or 1.5 ". Bench measurements resulted in a resonant frequency of $2856 \pm .3 \mathrm{MHz}, Q_{L}$ of 1200 and $\beta \equiv Q_{w} / Q_{e} \approx 7.0 \quad[66]$.

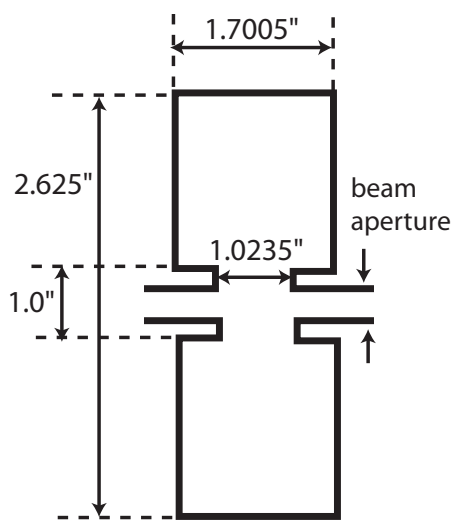

Figure 3.6: $\phi$-cavity dimensions.

The rectangular cavities, known as the x-cavity and y-cavity, were operated in the dipole modes $T M_{210}$ and $T M_{120}$, respectively. In the dipole mode the cavity is linearly dependent on charge and position, so after charge normalization it can be used as a position monitor. The dimensions of the x-cavity are given in Figure 3.7. The y-cavity has the same dimensions, except that length and height are interchanged. As in the case of the $\phi$-cavity the beam aperture was $0.8 ", 1.5 "$ or $2 "$. The resonant frequencies of these cavities were measured with a network analyzer. Their values depended on temperature due to the thermal expansion or contraction of cavity walls. Bench measurements resulted in a temperature dependence of resonance of $\approx-50 \mathrm{kHz} /{ }^{\circ} \mathrm{C}$. Such dependence meant that BPM temperatures had to be kept constant to within 1-2 ${ }^{\circ} \mathrm{C}$, to satisfy E158 experimental requirements. A water cooling system was used to stabilize the temperatures.

To improve the tune, triple stub tuners were added to the position cavities. The tuners modified the resonant frequencies by a few hundred $\mathrm{kHz}$ with a precision of the order of kHz, and increased the cavities' $Q_{L}$. Furthermore, circulators were attached 


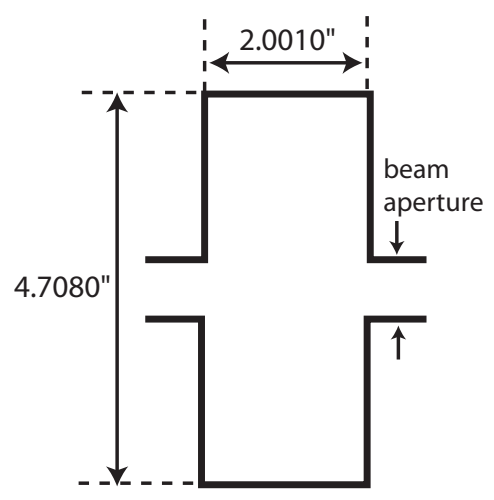

Figure 3.7: x-cavity dimensions.

to the tuner outputs (Figure 3.8), with port one of the circulator connected to the tuner and port two to the BPM processors, located in the counting house. During normal running the third port was terminated. However, a network analyzer could be connected to the second and third ports, in order to check the tune remotely, a very useful feature when running under high radiation conditions.

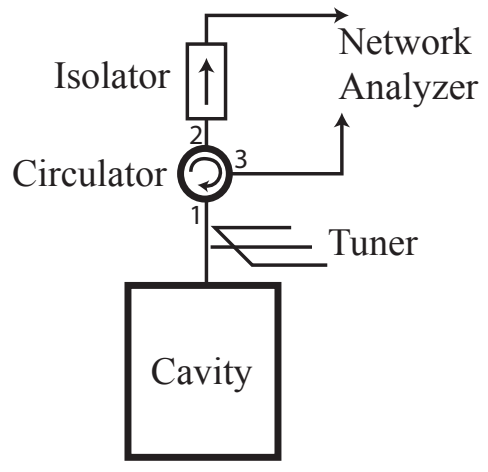

Figure 3.8: Cavity setup.

Since the beam raises the temperature by a few degrees $\mathrm{C}$, position cavities were tuned to a frequency 100-200 kHz higher than the $2856 \mathrm{MHz}$ value. While a high Q was desirable, the resonance band had to be wide enough to allow for small detuning 
or for temperature fluctuations. As a result the BPMs were set at $\beta \approx 1.15-1.3$, which corresponded to a $F W H M \approx 1 M H z$, and a $Q_{L} \approx 3000$.

The further the beam is offset from the center of the position cavities, the smaller the resolution, since increased contributions from higher-order components in Eq. 3.10 arise. The E158 BPMs were mounted on stages with two degrees of freedom, so that BPMs could be aligned in the $\mathrm{x}$ and $\mathrm{y}$ direction (here $\mathrm{z}$ is defined as the direction of the beam). The alignment precision of $0.1 \mathrm{~mm}$ was adequate.

\subsubsection{Electronics}

The radio frequency (rf) signals picked up by the BPMs were transmitted via coaxial cables to the BPM processors. As seen in Eqs. 3.6 and 3.7, these signals contain a fast oscillating part with frequency $\Omega_{\lambda}=2 \pi \times 2856 \mathrm{MHz}$, as well as an exponentially decaying envelope, whose integral is dependent on beam position and charge. Inside the processors the rf input was mixed with a signal in phase with the beam coming from a Local Oscillator (LO). This resulted in an output stripped from the fast oscillations, containing only the exponentially decaying envelope. After integration by the 16-bit ADCs the remaining, digitalized output was proportional to charge and position.

The LO signal used by each BPM processor originated from a power distribution chassis (Figure 3.9). One of the beam harmonics, a $476 \mathrm{MHz}$ signal provided by the main-drive line, was multiplied by 6 with a phase multiplier to create a 2856 $\mathrm{MHz}$ signal in phase with the beam. This signal was then amplified to $\sim 30 \mathrm{dBm}$ by a limiting amplifier, and divided through a series of splitters into outputs approximately equal in power, one for each cavity of a BPM. During the experiment, the LO power was monitored continuously with a power meter.

The BPM processor chassis (Figure 3.10) had two inputs: the LO from the power distribution chassis, and the rf from a BPM cavity. The rf input was attenuated by a variable attenuator, giving flexibility for setting the dynamic range of the BPMs. In addition, the rf signal was fed to a $180^{\circ}$ phase shifter, a bandpass filter, and 


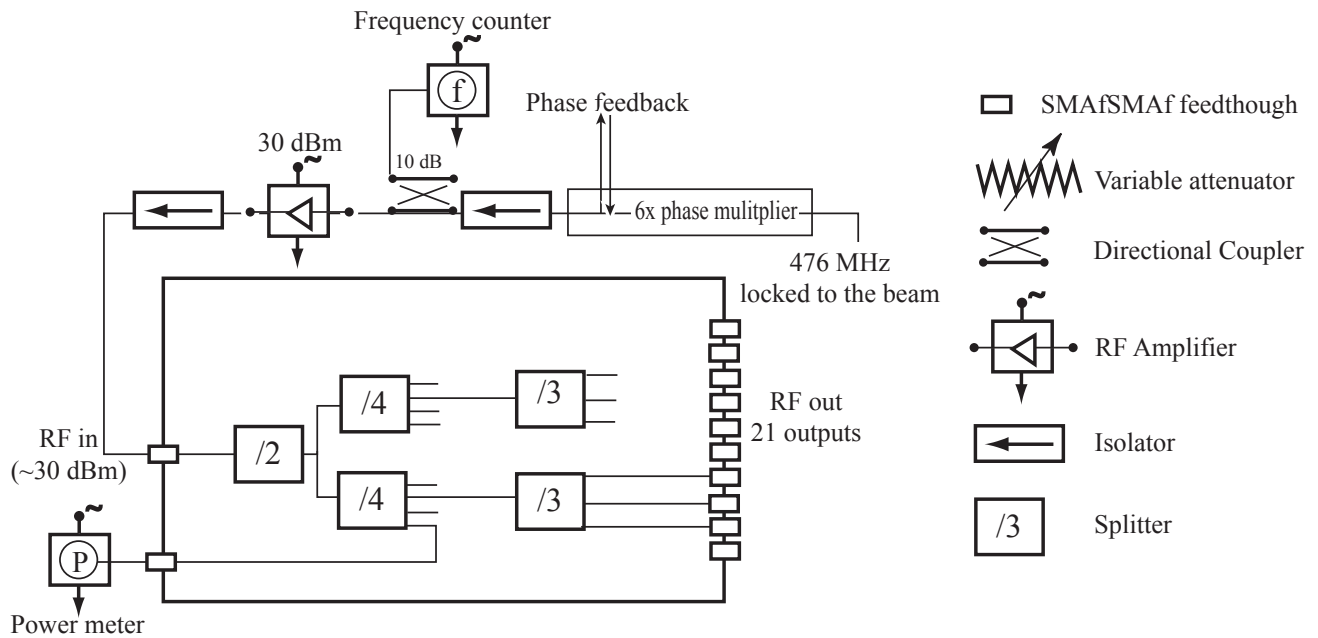

Figure 3.9: Power distribution chassis.

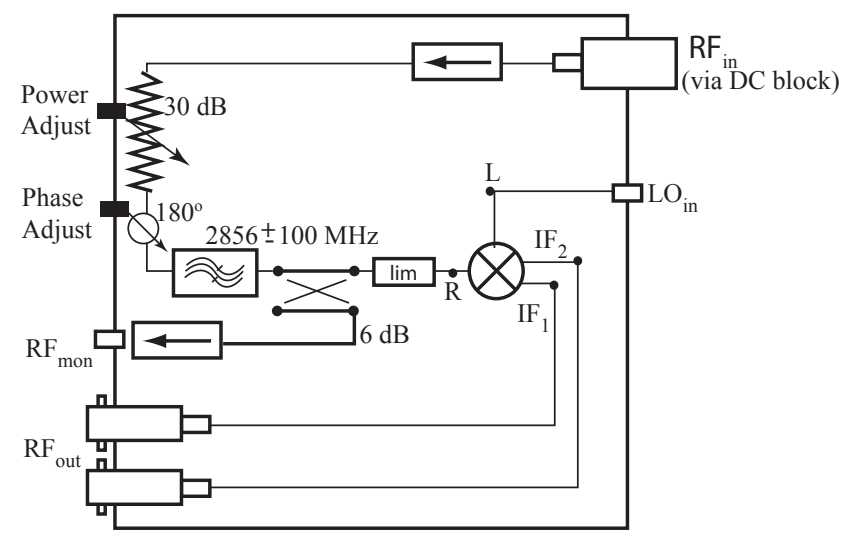

NfSMAf feedthough
SMAfSMAf feedthough

Figure 3.10: BPM processor. 
a limiter. A directional coupler split a small fraction of the signal for monitoring purposes. Finally, both the rf and the LO were sent to a quadrature IF mixer (QIF), which demodulated the signal. If the mixer input signals are $R F_{\text {input }} \propto A e^{i\left(\omega_{1} t+\phi\right)}$ and $L O_{\text {input }} \propto B e^{i \omega_{2} t}$, where $\phi$ is the phase difference between the two, the QIF mixer "multiplies" these two inputs to produce two equal-amplitude IF outputs: $I F_{1} \propto$ $A B e^{i\left[\left(\omega_{1}-\omega_{2}\right) t+\phi\right]}$ and $I F_{2} \propto A B e^{i\left[\left(\omega_{1}-\omega_{2}\right) t+\phi+\pi / 2\right]}$. In our case $\omega_{1}=\omega_{2}=2 \pi \times 2856$ $\mathrm{MHz}$, therefore the real parts of the outputs were just $I F_{1} \propto A B \cos (\phi)$ and $I F_{2} \propto$ $A B \sin (\phi)$. As a result, the fast oscillations were removed.

The amplitude of the mixer outputs had a simple linear dependence on position. To obtain just the amplitude, the phase difference $\phi$ was adjusted with the $180^{\circ}$ phase shifter until one of the IF outputs vanished, while the other one equaled the amplitude. However, during the course of the experiment, phase drifts occurred, mostly due to temperature variations. These phase drifts were minimized by adding a phase feedback to the power distribution chassis (Figure 3.9).

$I F_{1}$ and $I F_{2}$ outputs for each cavity were fed to integrating ADCs. Figure 3.11 shows what these signals looked in the oscilloscope. They start to decay exponentially after $\sim 270$ ns, which was the length of the bunch train. From Equation 3.7 one finds the decay constant to be: $\frac{1}{2} \nu_{\lambda}=\frac{1}{2} \frac{\omega_{\lambda}}{Q_{L \lambda}}$, where $\omega_{\lambda}=2 \pi \times 2856 \mathrm{MHz}$. An exponential fit demonstrated in Figure 3.11 allows one to determine that BPM cavities had $Q_{L} \approx$ 3000 .

\subsubsection{Position Measurement}

SLAC experiment E158 used nine BPMs shown in Figure 3.12. The first three, with beam aperture $=0.8 "$, were positioned in the ASSET region, where the beam energy was only $1.2 \mathrm{GeV}$, and its spot size $\sim 50 \mu \mathrm{m}$. The following four BPMs, with aperture=2", were located in the A-Line. Of those four BPMs, the two upstream ones, known as "energy BPMs", were located in a region where the beam had a large dispersion. Therefore, position offsets were used to deduce the beam energy offsets ${ }^{3}$.

\footnotetext{
${ }^{3}$ The dispersion relation is given by: $\Delta X=\eta \frac{\Delta E}{E}$, where $\eta=50 \mathrm{~cm}$ is the dispersion. Knowing the beam offset from the center of the cavity $(\Delta X)$ and the beam energy $(\mathrm{E})$, one can measure small
} 

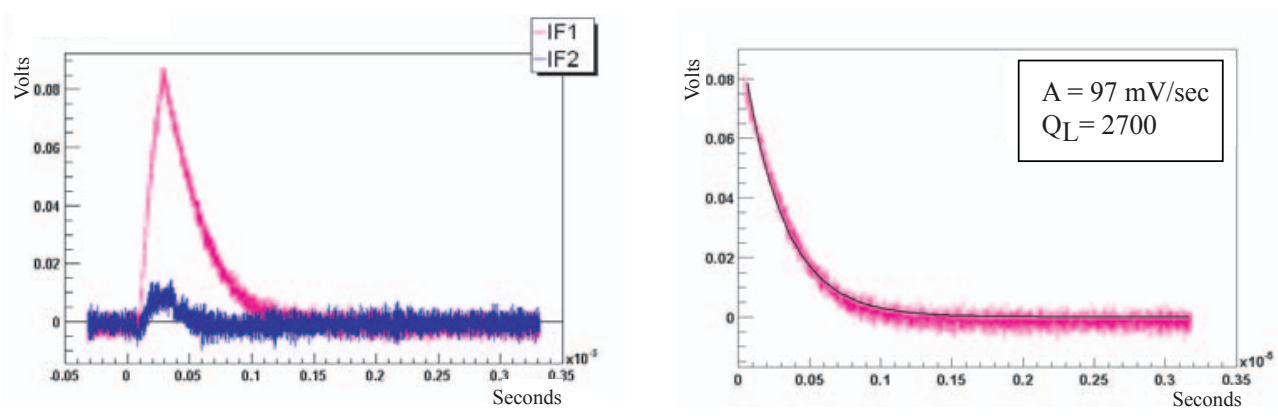

Figure 3.11: On the left: $I F_{1}$ and $I F_{2}$ waveforms after phase adjusts. On the right: Fitting $A \cdot e^{-\frac{\pi \cdot 2856 \cdot 10^{6}}{Q_{L}} t}$ to the exponentially decaying part of $I F_{1}$ waveform.

The two downstream ones, known as "angle BPMs", were mounted at the very end of the A-Line. ESA housed the last two "position BPMs", placed $\sim 3$ m upstream of the target. The BPM furthest from the target had a 2" aperture, and the one closest had a 1.5" aperture. At this point the electron beam spot size was $\sim 1-1.5 \mathrm{~mm}$.

As was the case for toroids, pairing the BPMs added to the redundancy and enhanced the position resolution. Furthermore, one can calculate the angle by which the beam was hitting the target by comparing the results of the last two BPM pairs ("angle" and "position" BPMs), since there were no bending magnets in between. The $40 \mathrm{~m}$ distance between these pairs was sufficient to measure angle at a resolution of $0.1 \mu \mathrm{rad}$.

To obtain an absolute position calibration, the integrated, charge-normalized response of the BPMs was compared to devices, whose absolute calibration was known, but lacked the BPM precision. The device most commonly used for this purpose was the wire array, which is described in Section 3.3. The absolute calibration was further improved by implementing a relative calibration for adjacent BPMs. For example, if BPMs in a pair measured $x_{1}$ and $x_{2}$ in each x-cavity, position difference $\Delta_{x}$ for a energy variations $(\Delta E)$. 


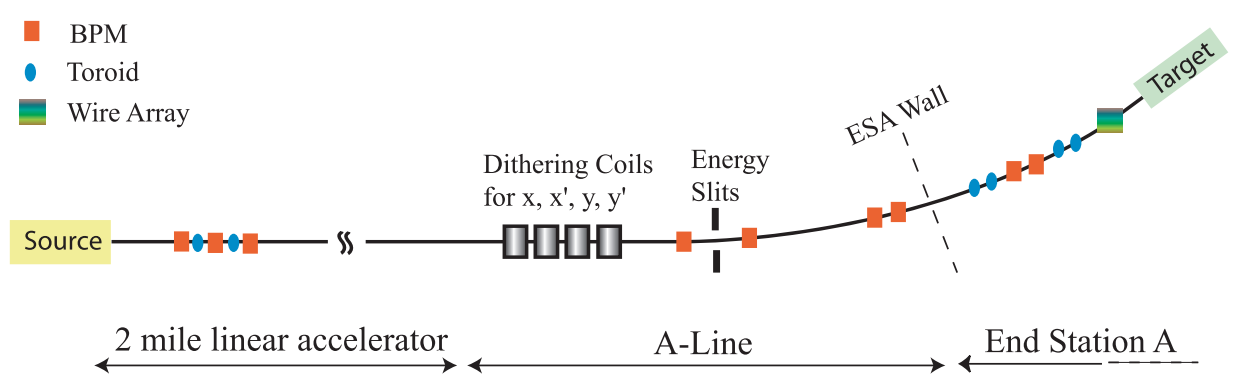

Figure 3.12: BPM placement along the beam line.

helicity pulse pair was defined as

$$
\begin{aligned}
& \Delta_{x}^{1}=x_{1}(\text { right_helicity })-x_{1}(\text { left_helicity }) \\
& \Delta_{x}^{2}=x_{2}(\text { right_helicity })-x_{2}(\text { left_helicity })
\end{aligned}
$$

A plot of $\Delta_{x}^{1}-\Delta_{x}^{2}$ versus $\Delta_{x}^{2}$ should be linear with a slope close to 0 . The difference (1-slope) would give the relative calibration in $\mathrm{x}$ of $\mathrm{BPM}_{2}$ compared to $\mathrm{BPM}_{1}$.

Once the calibrations, both absolute and relative, were complete, a comparison of $\Delta_{x}^{1}$ versus $\Delta_{x}^{2}$ for a BPM pair, or $\frac{1}{2}\left(\Delta_{x}^{1}+\Delta_{x}^{3}\right)$ versus $\Delta_{x}^{2}$ for a BPM triplet was done, and the slope $s$ from the resulting line was extracted. If the calibration was done correctly the slope had to be very close to one. The BPM resolution and agreement were given by the mean and sigma of a Gaussian fit of the residuals:

BPM pair: $\quad \frac{1}{2}\left(\Delta_{x}^{1}-s \cdot \Delta_{x}^{2}\right)$

BPM triplet: $\quad \frac{1}{\sqrt{3}}\left[\frac{1}{2}\left(\Delta_{x}^{1}+\Delta_{x}^{3}\right)-s \cdot \Delta_{x}^{2}\right]$,

where the factors of $\frac{1}{2}$ and $\frac{1}{\sqrt{3}}$ follow from the definition of $\Delta_{x}$.

The idea behind this algorithm was that it removed common beam noise, leaving 
only noise due to the readout electronics. Since the ADCs were limited by fixed pedestal noise (3 counts for BPM ADCs), the bigger the input signal, the better the resolution. However, QIF mixers saturated at about $300 \mathrm{mV}$, which forced a compromise between the resolution and the dynamic range of the BPMs. To set the dynamic range to the desired amount, the beam was offset by that particular amount and the variable attenuator (Figure 3.10) was adjusted, such that, after phase tuning, the $I F_{1}$ signal was at about $75 \%$ of what saturated the mixer. The dynamic ranges for the "angle BPMs" and "position BPMs" were set to $\pm 1.5 \mathrm{~mm}$ and $\pm 1.0 \mathrm{~mm}$, respectively, which was dictated by beam jitter and beam drifts.

\subsubsection{Results}

During beam test T-437 in the ASSET region in November 2000, the BPMs achieved a $900 \mathrm{~nm}$ and $700 \mathrm{~nm}$ resolution in $\mathrm{x}$ and $\mathrm{y}$, respectively. A small sample of the data is given in Figure 3.13, where the scatter plot of $\frac{1}{2}\left(\Delta_{1}+\Delta_{3}\right)$ versus $\Delta_{2}$ and the plot of residuals are shown.

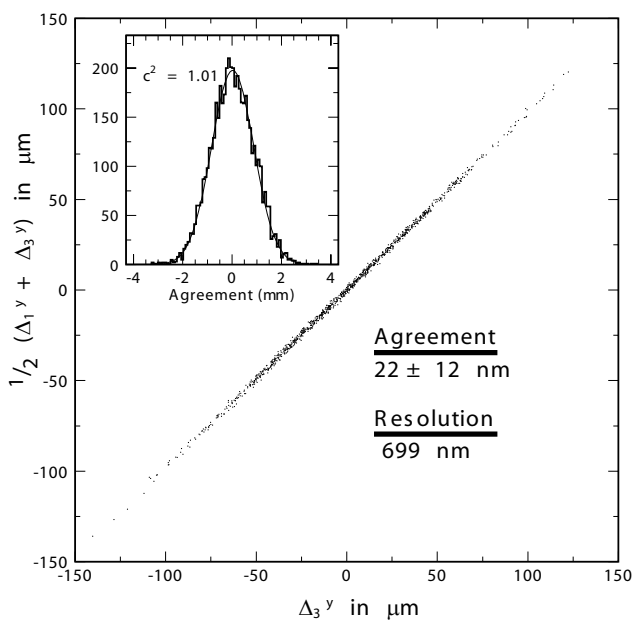

Figure 3.13: Resolution and agreement in y for the BPM triplet. The spread in the linear plot is due to beam jitter. A Gaussian fit of the residuals determines the BPM resolution and agreement.

The BPM performance during Run I and Run II varied between 1-4 $\mu \mathrm{m}$ resolution 
per pulse, as indicated in Table 3.1. While these resolutions were sufficient to satisfy the E158 running conditions, they were worse than the T-437 test for several reasons. First, beam conditions were less ideal having a bigger beam jitter (50-100 $\mu \mathrm{m})$, more drifts, and not a perfect tri-Gaussian beam pulse shape. Second, BPMs were set to a wider dynamic range in the A-Line and ESA, which lowered their resolution, as explained in Section 3.2.4. Third, temperature controls were less stringent in the A-Line and ESA than in the ASSET region. Temperature fluctuations had a twofold effect. They led to detuning from resonance due to thermal expansion of the cavities, as well as phase drifts, both of which made the position measurement less accurate and worsened the resolution. The BPM agreements averaged over an entire Run are given in Table 3.2. Within uncertainties the BPMs agreed.

\begin{tabular}{|l|c|c|}
\hline BPM Pair & Run I Resolution per pulse & Run II Resolution per pulse \\
\hline energy BPMs & $1-2 \mathrm{MeV}$ & $1-2 \mathrm{MeV}$ \\
\hline angle BPMs in $\mathrm{x}$ & $3-4 \mu \mathrm{m}$ & $2-4 \mu \mathrm{m}$ \\
\hline angle BPMs in $\mathrm{y}$ & $2-3 \mu \mathrm{m}$ & $3-4 \mu \mathrm{m}$ \\
\hline position BPMs in $\mathrm{x}$ & $1-2 \mu \mathrm{m}$ & $2-3 \mu \mathrm{m}$ \\
\hline position BPMs in $\mathrm{y}$ & $3-4 \mu \mathrm{m}$ & $2-4 \mu \mathrm{m}$ \\
\hline
\end{tabular}

Table 3.1: BPM resolutions per pulse during Run I and Run II.

\begin{tabular}{|l|c|c|}
\hline BPM Pair & Run I Agreement & Run II Agreement \\
\hline angle in $\mathrm{x}$ & $-3.3 \pm 1.0 \mathrm{~nm}$ & $0.42 \pm 0.87 \mathrm{~nm}$ \\
\hline angle in $\mathrm{y}$ & $0.91 \pm 0.61 \mathrm{~nm}$ & $-2.0 \pm 0.86 \mathrm{~nm}$ \\
\hline position in $\mathrm{x}$ & $0.95 \pm 0.55 \mathrm{~nm}$ & $-0.41 \pm 0.61 \mathrm{~nm}$ \\
\hline position in $\mathrm{y}$ & $-0.15 \pm 0.89 \mathrm{~nm}$ & $-2.5 \pm 0.88 \mathrm{~nm}$ \\
\hline
\end{tabular}

Table 3.2: BPM agreement averaged over Run I and Run II.

\subsection{Wire Array}

The beam spot size and higher-order moments were measured with the wire array. The wire array consisted of a grid of $0.18 \mathrm{~mm}$ in diameter $\mathrm{Cu}$-Be wires, placed 0.36 $\mathrm{mm}$ apart. As the beam passes through, it ionizes the wires, with the ionization level depending on the beam current density. One can reconstruct the beam profile by 
measuring the voltage across each wire. The signal from the wires was read by a standard CAMAC 2219W 11-bit ADC. The wire thickness and separation limited the wire array resolution to $\sim 13 \mu \mathrm{m}$. During data collection the wire array results were displayed online. A typical online histogram is given in Figure 3.14, where the mean of the profiles determines the beam position, while the root mean square characterizes the beam jitter.
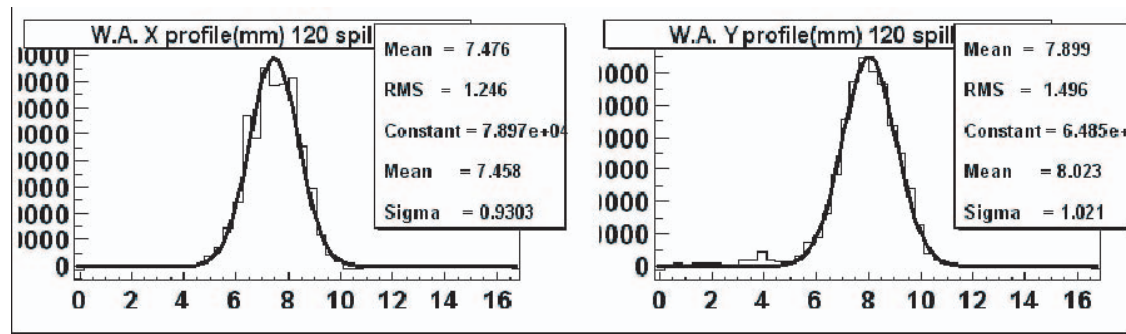

Figure 3.14: Wire array display when averaged over 1 second.

During the first half of Run I, the wire array was continuously in the beam. Unfortunately, radiation damage to the wires ruined the device for the later part of the Run. The wire array was repaired for Run II, during which it was inserted periodically to what amounted to $5 \%$ of all production runs. The device was also used occasionally for beam steering and calibration.

\subsection{Synchrotron Light Monitor}

As electrons are bent into the A-Line, they emit synchrotron radiation whose power is proportional to $E^{2} B^{2}$, where $B$ is the magnetic field of the dipole and $E$ is the beam energy [68]. Measuring the synchrotron radiation asymmetry provided an additional monitor for estimating beam energy fluctuations. The Synchrotron Light Monitor (SLM) was positioned in the A-Line, on the left of the beam pipe when looking upstream of the beam. From Figure 3.15 one can see that the main SLM components 
consisted of an aluminum flange followed by a lead filter/radiator and a quartz radiator, which served to convert the $1 \mathrm{MeV}$ synchrotron radiation into visible light [63]. The light was sent though a lightguide and a system of mirrors into three UDT PIN 10D photodiodes surrounded by lead shielding, with a fourth photodiode measuring the background outside the shielding. Finally, the photodiode signals were read by a CAMAC 2219W 11-bit ADC.

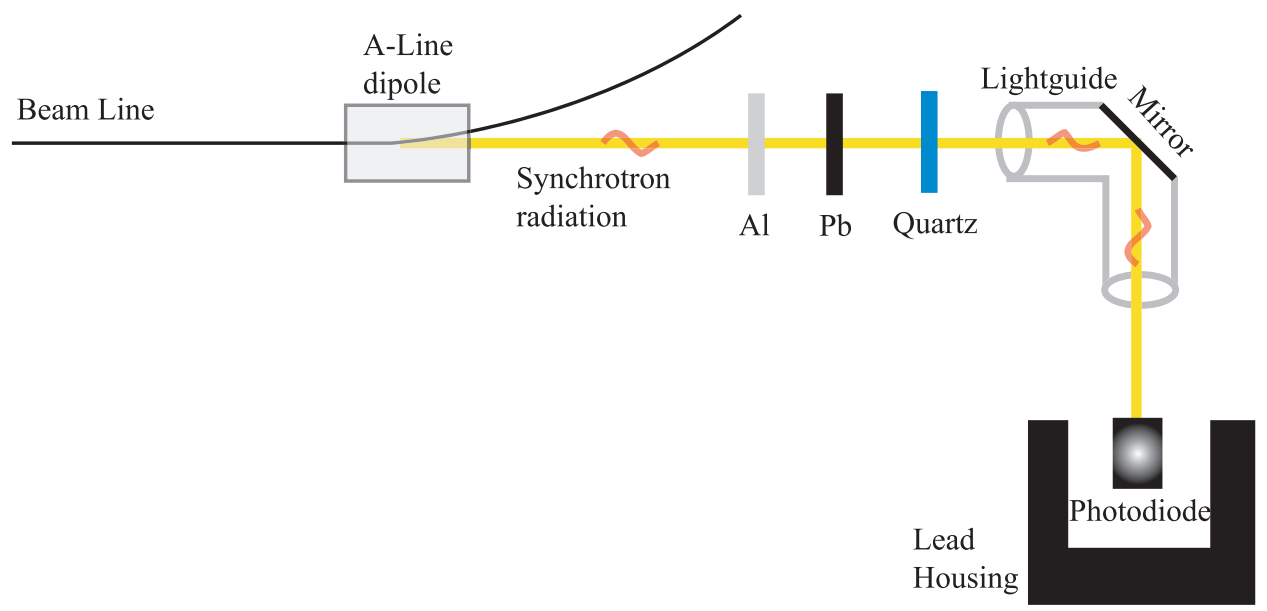

Figure 3.15: Top view schematic of the Synchrotron Light Monitor. 


\section{Chapter 4}

\section{Target and the Spectrometer}

In January 2000 End Station A (ESA) was an empty experimental hall. After one year of intensive construction, the $60 \mathrm{~m}$ long hall was occupied by a target, an electron spectrometer, a detector package and concrete blocks surrounding the entire setup as a radiation shield. The layout of ESA is given in Figure 4.1. This chapter contains a description of the target and the spectrometer, both designed and built specifically for Experiment E158.

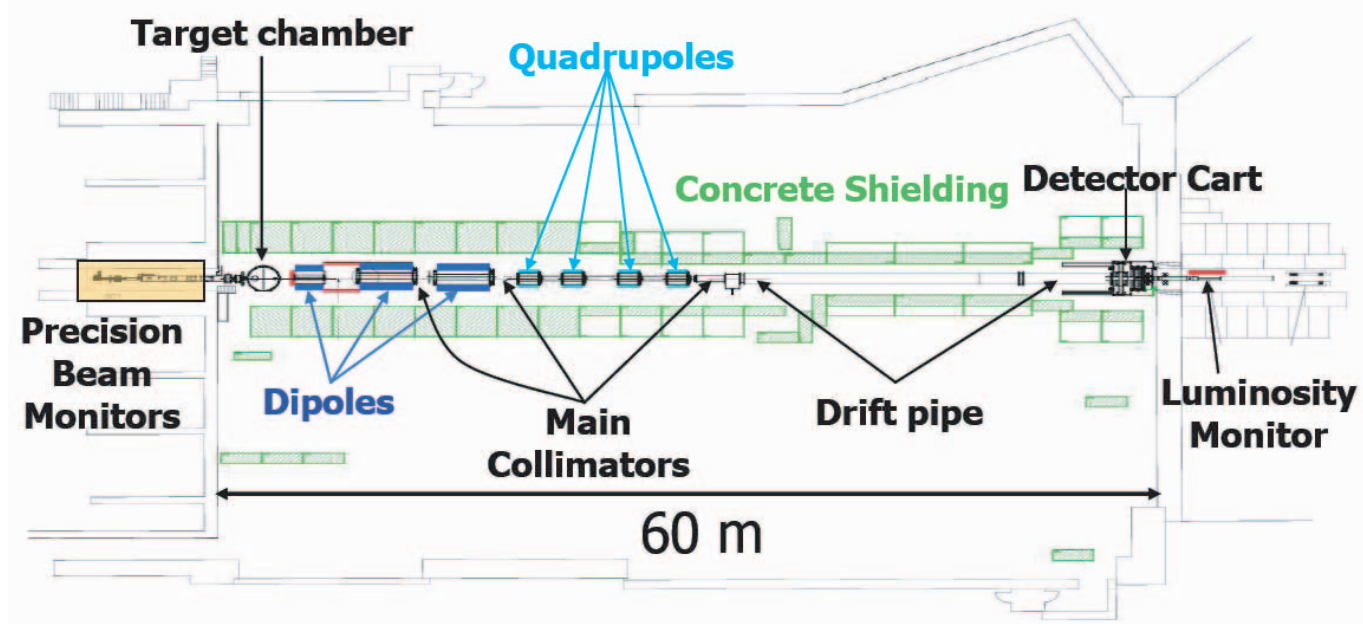

Figure 4.1: The layout of End Station A. 


\subsection{Liquid Hydrogen Target}

Experiment E158 used a liquid hydrogen target, since it is the most practical electron target, given its high electron/proton ratio and minimum radiation length per target electron. The target consisted of a $1.5 \mathrm{~m}$ long and $76.2 \mathrm{~mm}$ inner diameter cylindrical aluminum cell filled with liquid hydrogen, maintained at a temperature of $18 \mathrm{~K}$ [22]. This length corresponds to 0.17 radiation lengths (r.l.) and 0.21 interaction lengths (i.l.) of liquid hydrogen. The target cell was part of a closed loop system which, in addition, contained a pump, a heat exchanger and a heater, as shown in Figure 4.2. The pump ensured that the hydrogen flowed through the loop. The heat exchanger consisted of a copper tube, surrounded by a stainless steel shell. As hydrogen circulated through the shell, cold helium circulated in the opposite direction within the tube, thus cooling the hydrogen. The heater was used to maintain a constant heat load on the target independent of the beam power. For this purpose, the heater was controlled by a feedback loop adjusted by the electron beam current. Given that the beam could deposit a heat load of $\sim 700 \mathrm{~W}$ on the target and taking into account various heat leaks, the heater was designed to provide as much as $1000 \mathrm{~W}$ of power.

An important aspect of the target design was minimizing pulse-to-pulse hydrogen density fluctuations, which would have led to an artificial increase of the width of the experimental asymmetry distribution. The minimization was achieved by sustaining a high hydrogen flow rate of $10 \mathrm{~m} / \mathrm{s}$, and by installing eight wire mesh disks inside the target cell, perpendicular to the direction of the beam. These disks introduced turbulence and transverse flow within the target cell, helping to maintain the hydrogen at uniform density throughout the cell. As a result of these measures, the target density fluctuations contributed $<70 \mathrm{ppm}$ to the experimental asymmetry distribution width [69].

The target loop was enclosed in a spherical aluminum scattering chamber (Figure 4.2) kept at room temperature and at a vacuum of $10^{-8}-10^{-9}$ Torr. By providing the target loop with vacuum insulation, the scattering chamber acted as a cryostat. The target loop hung from an external frame, linked to motorized jacks, which al- 


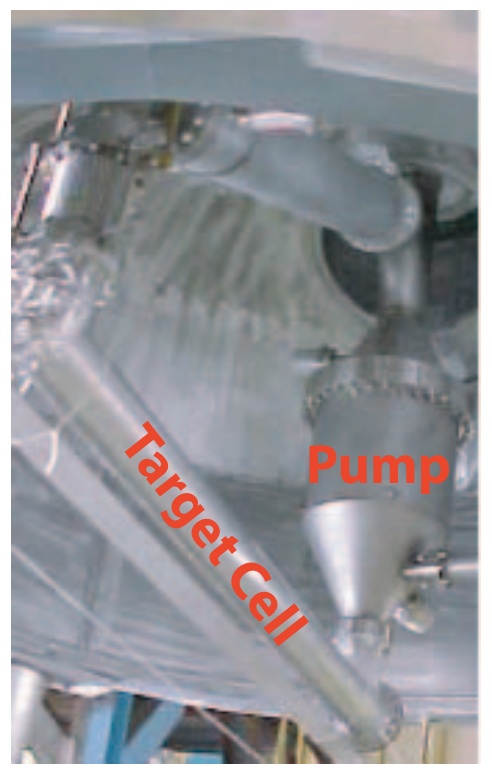

(a)

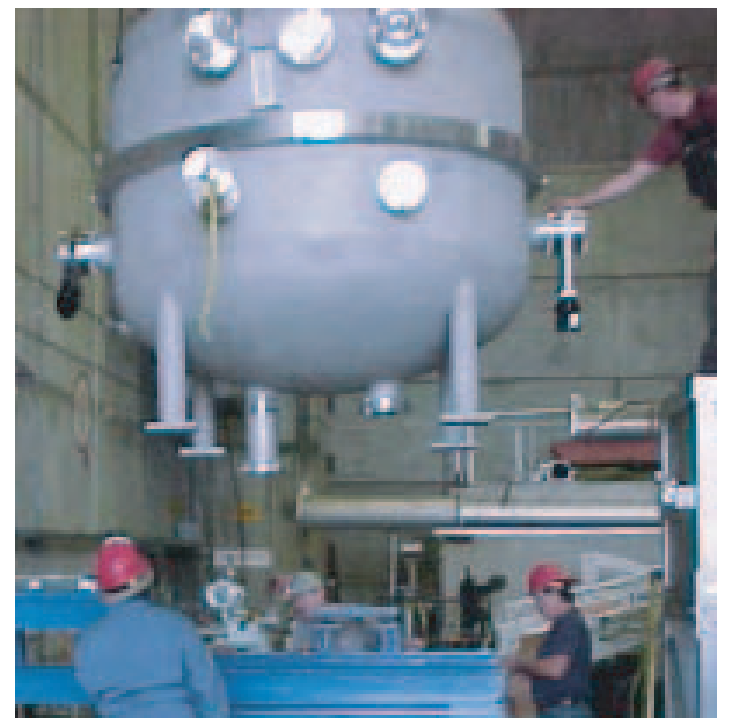

(c)

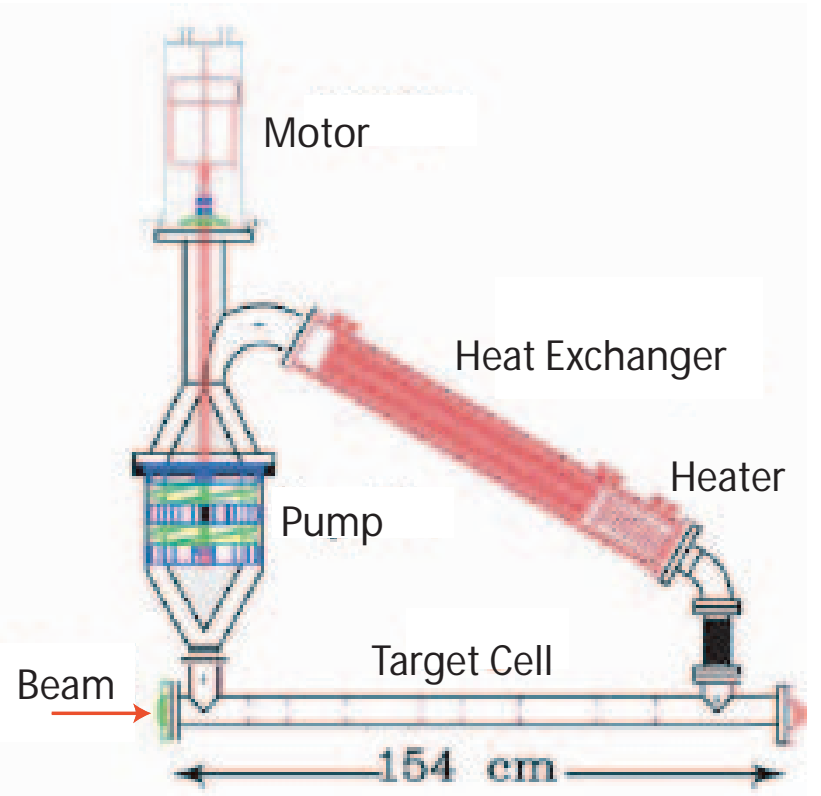

(b)

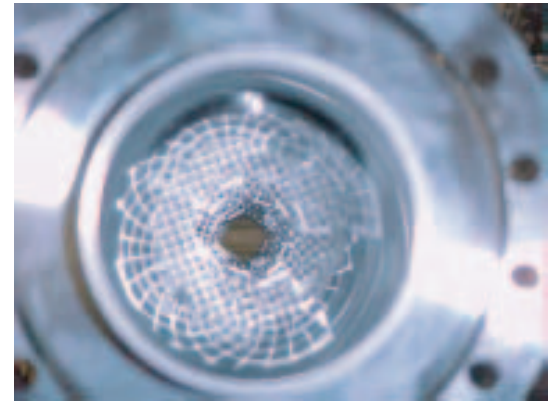

(d)

Figure 4.2: The liquid hydrogen target. (a) Photograph of the target cell and the pump. (b) Schematic of the target loop. (c) Photograph of the scattering chamber. (d) Photograph of the wire mesh disks. 
lowed for target motion $15 \mathrm{~cm}$ in and out of the beam line. Additionally, the scattering chamber contained a laterally insertable table of carbon targets. These targets, ranging in thickness from $30 \mu \mathrm{m}$ to $8 \mathrm{~cm}$, were used for spectrometer and detector calibration. Both the hydrogen and the carbon target movement was controlled remotely from the experimental counting house.

Safety was one of the most important aspects of designing and operating the target. The 55 liters of liquid hydrogen contained in the target are equivalent to 8 $\mathrm{kg}$ of TNT. A system of vents and safety procedures were introduced in the event of excessive pressure in the target loop. Furthermore, the hydrogen temperature and pressure were monitored continuously during the experiment. A LabView program was used as a target readout and as a mean of implementing computerized feedback loops, which kept the target temperature constant to within $0.1 \mathrm{~K}$.

\subsubsection{Foil Target}

The foil target was used for beam polarimetry measurements and was positioned immediately upstream of the hydrogen target. It contained three supermendur ${ }^{1}$ foils of thicknesses of 30, 50 and $100 \mu \mathrm{m}$, enclosed by two Helmholtz coil magnets. The foils were mounted at a sixty degree angle with respect to the beam along the $y-z$ plane, as shown in Figure 4.3, and could be remotely inserted in and out of the beam line. During the polarimetry measurement the current in the Helmholtz coils was set to 6 Amps, producing a field of $\sim 90$ gauss, which polarized the supermedur near saturation. The polarimetry measurement is described in Section 5.4

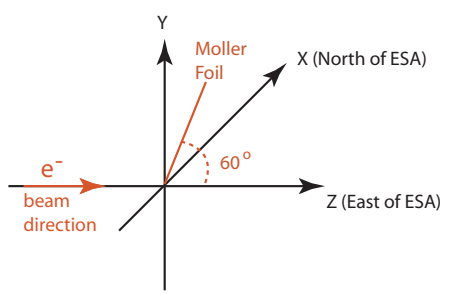

Figure 4.3: The orientation of the polarimetry foils with respect to the beam.

\footnotetext{
${ }^{1}$ Supermendur is a $50 \%$ iron $-50 \%$ cobalt alloy.
} 


\section{$4.2 \quad$ E158 Spectrometer}

The E158 spectrometer was designed to satisfy the following criteria:

- Acceptance along the entire azimuth of scattered Møller electrons in the momentum range of $12-24 \mathrm{GeV} / \mathrm{c}$.

- Separation between Møller and Mott electrons.

- Reduction of photon background.

- Placement of detectors out of the "line of sight" of the target.

- Use of aluminum, copper, tungsten or stainless steel for any component of the spectrometer that might see incident particle flux.

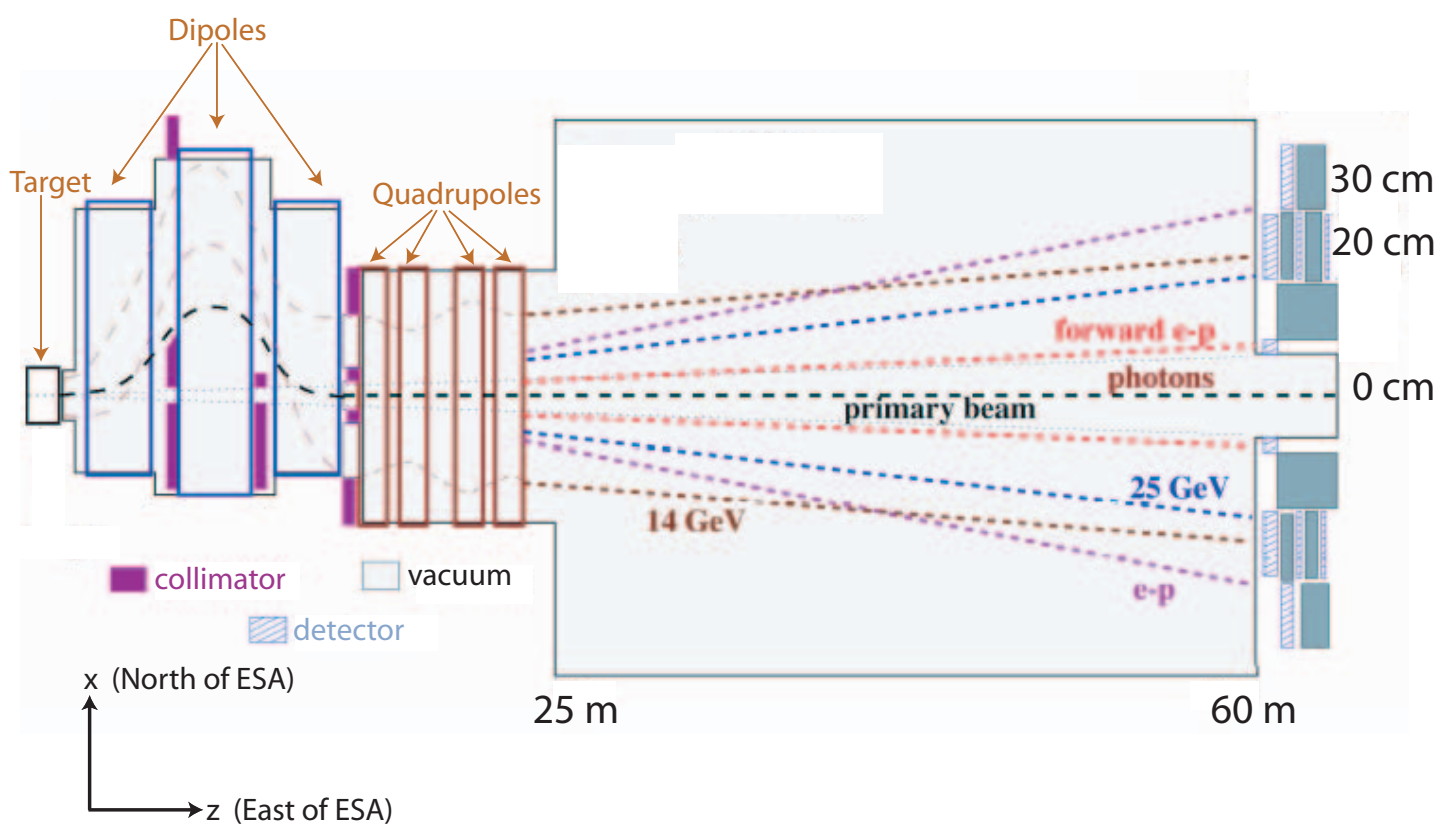

Figure 4.4: Top view schematic of the spectrometer and detector layout. The scale along the $\mathrm{z}$ direction is in meters, while the scale along the $\mathrm{x}$ is in centimeters.

The spectrometer consisted of a dipole chicane, followed by four quadrupole magnets and a drift pipe. A schematic of the spectrometer layout is shown in Figure 4.4. 
The z-direction is taken to be the direction of the beam. The positive $\mathrm{x}$ direction is taken to be to the left side of the spectrometer when looking downstream of the beam.

\subsubsection{Dipole Chicane}

The dipole chicane and the collimator setup were designed to place the Møller detector out of the line of sight of the target and to reduce "soft" backgrounds (low momentum particles, consisting of photons, positrons and electrons). The chicane blocked most of the target photon background by creating a "two-bounce" system, that is, a photon could reach the detector only after bouncing twice off the beam pipe or collimators [10]. The dipoles used for the chicane required large apertures to fit the full beam profile. They required fields strong enough to bend the electron beam centimeters away from the beam axis, so that photons could be collimated (Figure 4.4). Finally, the dipoles had to produce a uniform field on the order of $\pm 1-2$ Tesla, to keep the Møller signal profile at the detectors azimuthally symmetric. In the E158 spectrometer, three existing SLAC dipoles were used (18D72, B81 and B82,) all of which satisfied the above criteria [51].

To maintain an azimuthally symmetric Møller flux, the dipole fields were set to produce a zero net amount of transverse magnetic field on charged particles $\left(\int B_{\perp} d L=0\right)$, when integrated over all three dipoles. To ensure that the fields were stable, the current through each magnet (dipoles and quadrupoles) was continuously monitored. Audible alarms were activated if the current varied by more than $0.1 \%$ of the set value. The current for the magnets was provided by high voltage supplies, located in a building adjacent to ESA. To prevent overheating, all the magnets were water cooled. Additionally, water cooled copper masks were placed in the second and third dipoles to protect in case of a mis-steered beam and from synchrotron radiation. 


\subsubsection{Photon Collimators}

If the undeflected, line of sight photons were to hit the Møller detector, they would cause energy fluctuations ruining its resolution. Two cylindrical collimators were used to block these photons. Both were made out of 40 r.l. thick tungsten, surrounded by 40 r.l. copper in order to minimize "punch-through" leakage. The first collimator, known as 3DC2C, was located immediately upstream of the second dipole and absorbed most of the photon flux power, approximately two kilowatts (Figure 4.5). The second collimator, known as 3DC3, was located immediately downstream of the second dipole and served to reduce the radius of the photon beam to be small enough so that all the remaining photons would only hit the beam dump. Both collimators had their own water cooling line.

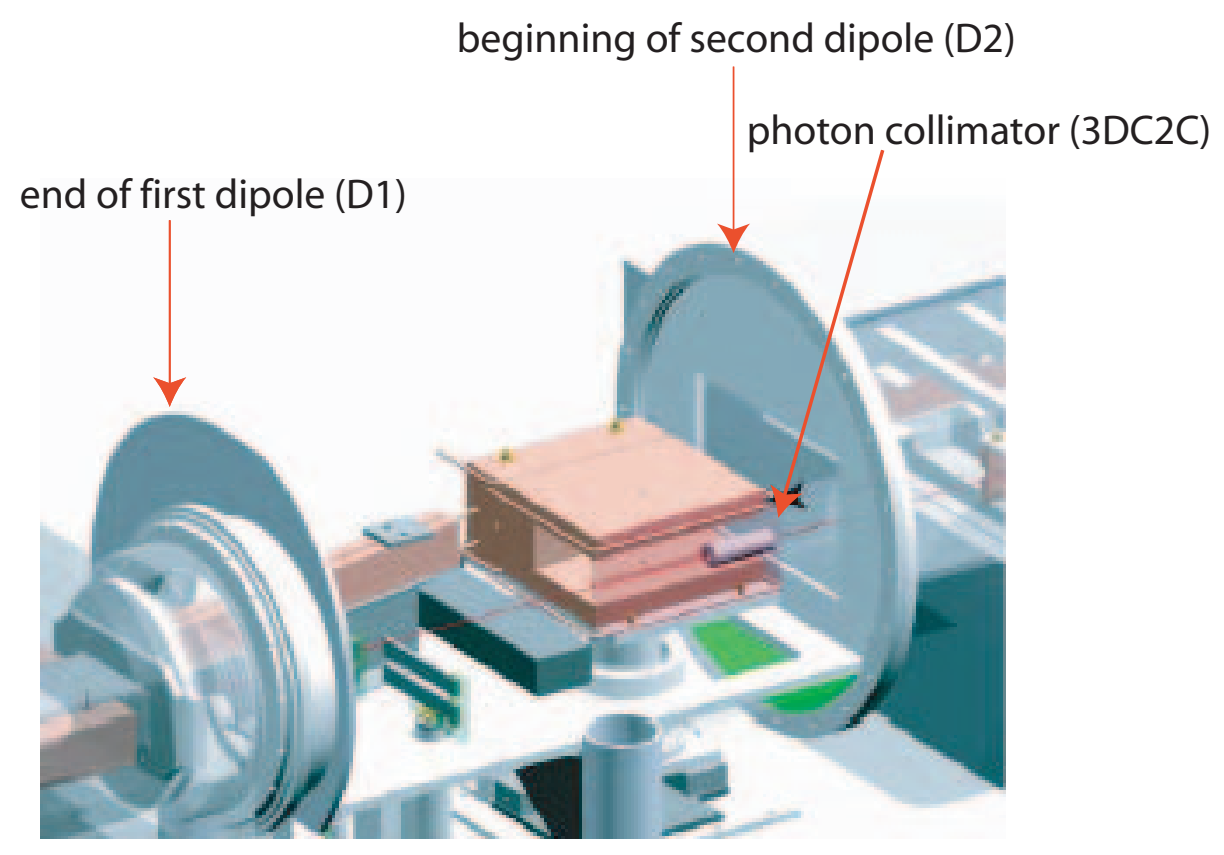

Figure 4.5: AutoCAD rendering of the first photon collimator. 


\subsubsection{Momentum Collimator}

The momentum collimator was used together with the quadrupoles to separate the Møller scatters from the Motts (ep scatters). The collimator, labeled as 3QC1B, was installed between the last dipole and the first quadrupole. It consisted of two concentric cylinders, connected by two horizontal "spokes". Each cylinder was 40 r.l. long and was made of a piece of tungsten brazed to a piece of copper. As one can see from Figure 4.6 the momentum collimator had two concentric openings. The outer opening served as a radial cut, corresponding to a momentum cut for the Møller and ep electrons. The inner opening, on the other hand, was large enough to allow high energy Møller and ep electrons to strike the luminosity detector without scraping the inner edge of the Møller detector. The inner opening also allowed for the passage of the primary beam to the beam dump.

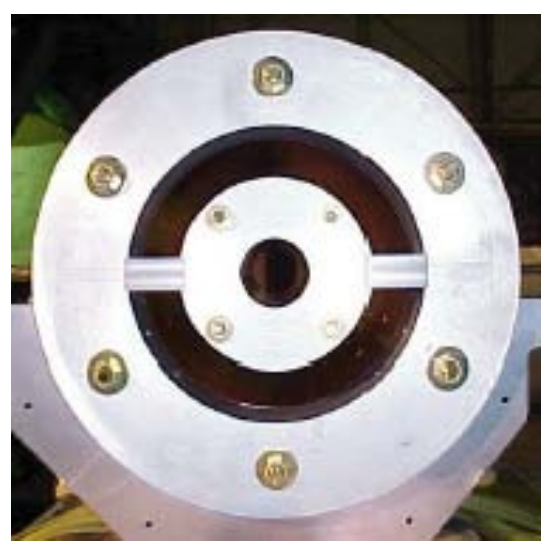

(a)

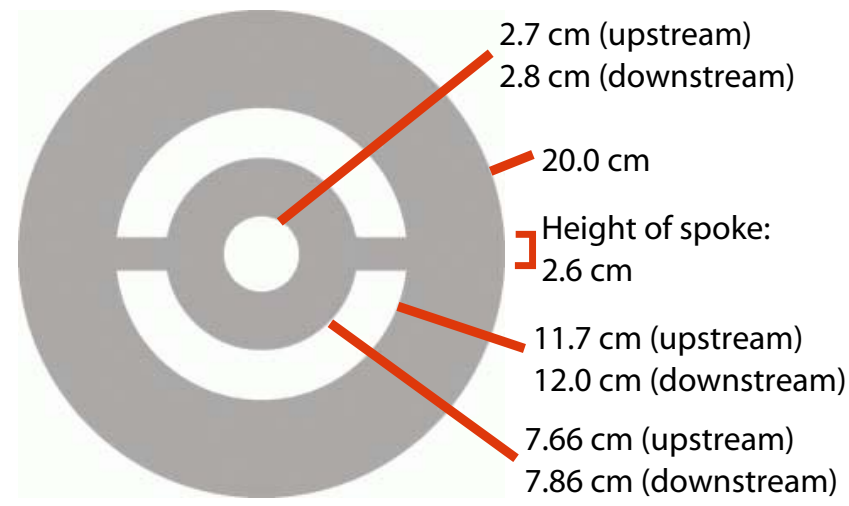

Piece thickness: $12.0 \mathrm{~cm}$ copper, $3.0 \mathrm{~cm}$ tungsten

(b)

Figure 4.6: (a) Photograph of the momentum collimator. (b) Upstream and downstream dimensions of the momentum collimator.

The dimensions of the collimator cylinders were chosen to maximize the separation between the Møller and the ep flux at the Møller detector, while at the same time maximizing the number of Møller electrons directed at the detector acceptance. All 


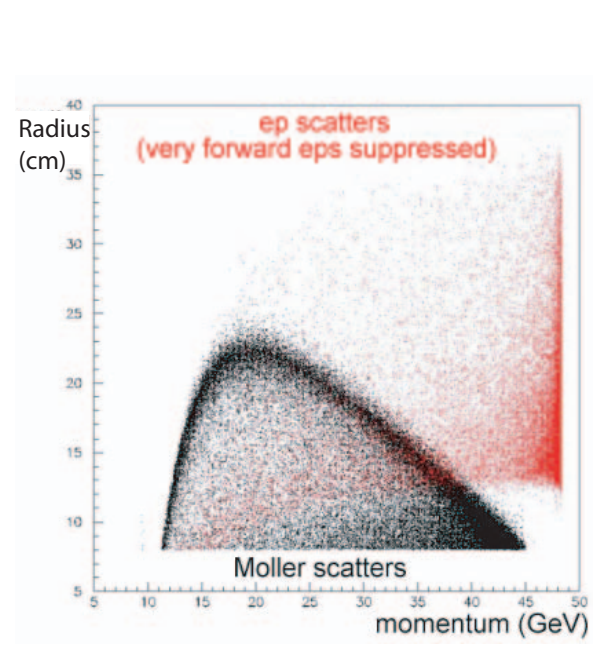

(a)

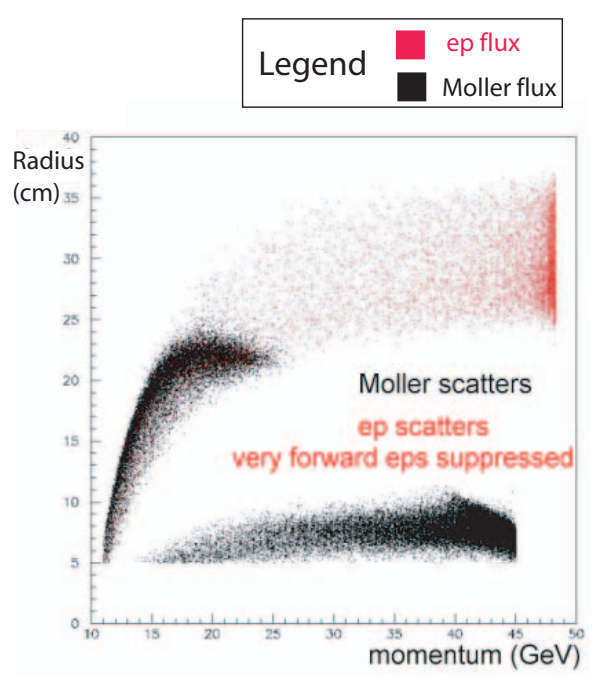

(b)

Figure 4.7: Simulations of the phase space of the Møller and ep flux at the Møller detector. (a) Without the momentum collimator. (b) With the momentum collimator.

collimator edges flared radially inward or outward to account for beam spread and to minimize particle scattering by the inner walls of the collimator. The effect of the momentum collimator on the Møller and the ep flux is shown by the simulations in Figure 4.7. Without the collimator, a large portion of the ep flux hits the detector at the same radii as the Møller flux. Having the collimator causes a clean radial separation between the Møller and the ep electrons.

\subsection{4 "Holey" Collimator}

The "holey" collimator was a remotely insertable device, used to study the inelastic ep flux in detail. The collimator, labeled as 3QC1A, was located immediately upstream of the momentum collimator. Its shape was a mirror image of the momentum collimator, consisting of two semi-cylindrical shells (Figure 4.8). When inserted, the shells blocked the entire outer acceptance region of the momentum collimator, while leaving the inner opening free. The reason for not designing the "holey" collimator as cylinder and, consequently, blocking the inner opening of the momentum collimator, 
was to decrease the beam power deposited into the device, thus avoiding the need for water cooling.

The cylindrical shells of the "holey" collimator had four $1 \mathrm{x} 1 \mathrm{~cm}$ holes cut through them. The holes were located at different radii, and were ninety degrees apart from each other. Their location was chosen to create a very clean separation between the Møller and the ep flux at the detector, when the collimator was inserted. The results obtained under these conditions were used as a model for estimating the inelastic ep flux during the experiment. In addition to the four "ep holes", there were two larger holes $(2 \times 2.6 \mathrm{~cm})$, which were useful for the polarimetry measurement, which will be described in Section 5.4

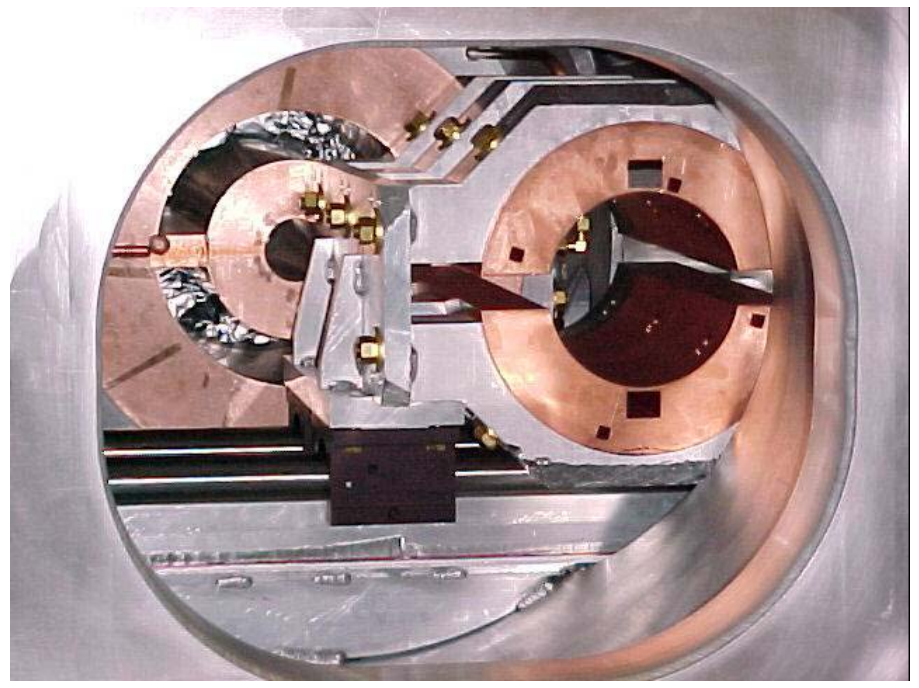

Figure 4.8: Photograph of the "holey" collimator 3QC1A (front) and the momentum collimator 3QC1B (back).

\subsubsection{Quadrupoles}

The E158 spectrometer made use of four existing SLAC quadrupoles (Q82, Q83, Q202, and Q203). These quadrupoles were remarkably uniform in magnetic field, with a field gradient variation of $<1 \%$ over a $19.4 \mathrm{~cm}$ radius [10]. The positions and strengths of the quadrupoles were optimized to achieve maximal separation between 
the Møller and the ep flux at the detector, while preserving their azimuthal symmetry. Furthermore, to prevent wide angle ep elastic flux from scattering off the walls of the last quadrupole, they were spaced to take as little room in the $\mathrm{z}$ direction as possible. Another crucial aspect of the quadrupole installation was their alignment. For this purpose, the quadrupoles were placed on top of precision jacks, which enabled coaxially aligning them to within $0.1 \mathrm{~mm}$. As a results, the Møller profile at the detector was azimuthally symmetric with a tolerance better than $1 \mathrm{~mm}$. Figure 4.9 depicts scans of the Møller and ep flux profiles with the quadrupoles on and off. One can conclude that the quadrupoles successfully separated the Møller from the ep flux.

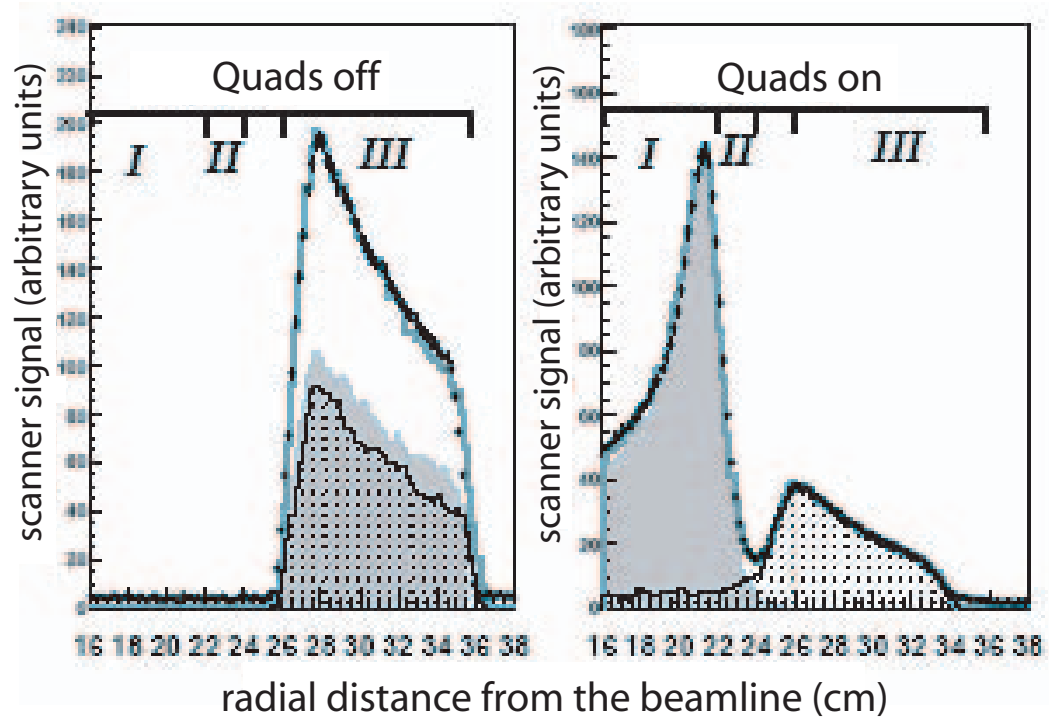

Figure 4.9: Scans of the flux profile at the detector with the quadrupoles on and off. The points represent the data scan and the open histogram is the Monte Carlo simulation. The Møller contribution is represented by a shaded histogram and the ep contribution by a hatched one. The parity-violating asymmetry was measured for regions I and III.

\subsubsection{Synchrotron Collimators and Collimator Masks}

Synchrotron radiation could potentially contribute to the experimental background. Monte Carlo simulations indicated that for these contributions to remain negligible the synchrotron flux had to be $<1 \%$ of the overall flux hitting the Møller detector [51]. 
To achieve this specification, three synchrotron collimators were used. The two horizontal spokes connecting the cylinders of the momentum collimator played the role of the first synchrotron collimator, since synchrotron radiation is produced in the horizontal plane only. The other two collimators, having the same shape and orientation as the first one, were installed immediately downstream of the fourth quadrupole and in front of the Møller detector, respectively. Both second and third collimators were made of 20 r.l. thick tungsten. The spokes in the first synchrotron collimator were chosen large enough to "shadow" the downstream synchrotron collimators. As a result the Møller and ep electrons that would have hit the two downstream collimators were stopped at the first one.

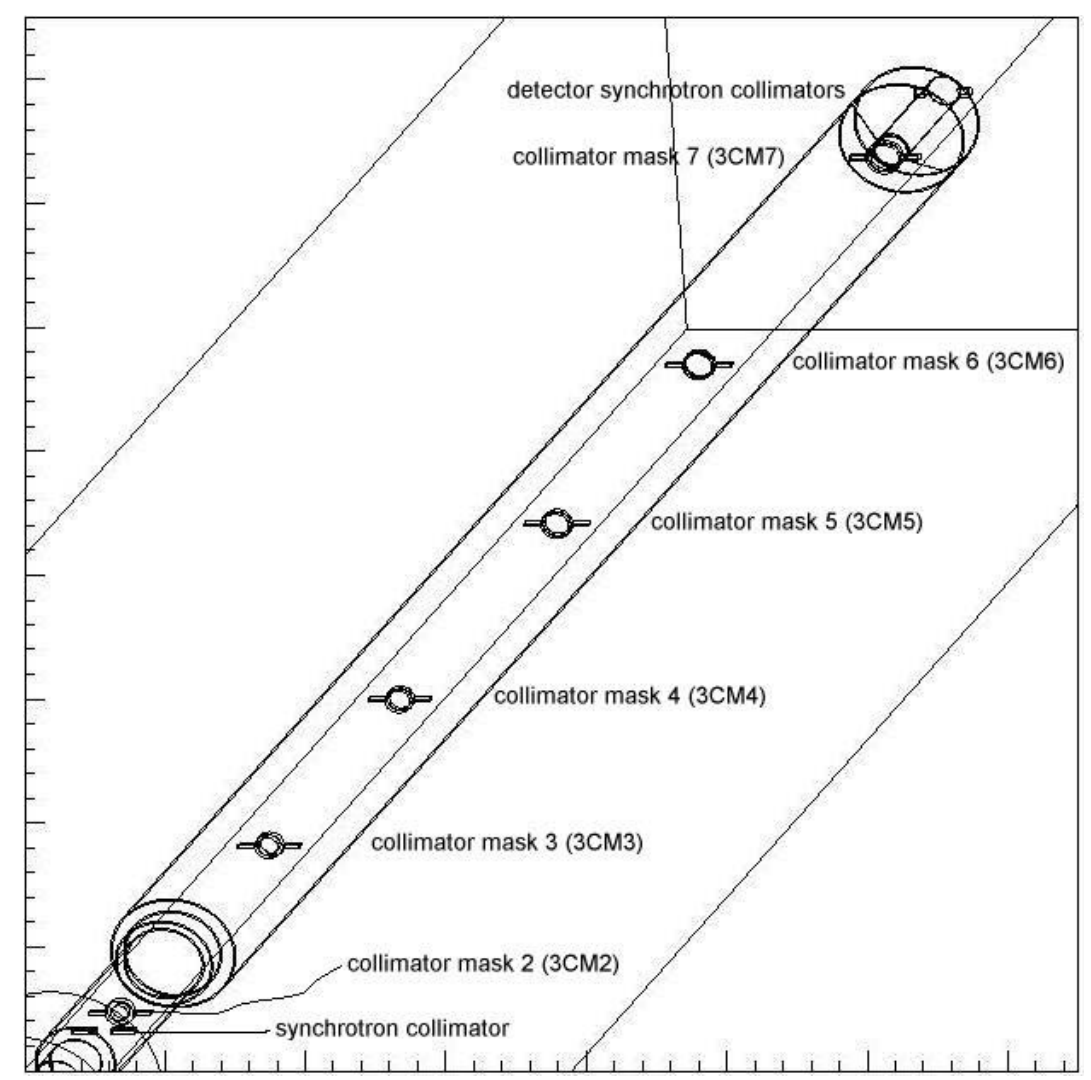

Figure 4.10: Schematic of the drift pipe containing the synchrotron collimators and the collimator masks.

After leaving the fourth quadrupole and continuing to the detector, the beam was enclosed by a wide aluminum drift pipe kept at vacuum in order to prevent 
air degradation. The two downstream synchrotron collimators were mounted inside the drift pipe. Between the two collimators, there were seven "collimator masks" consisting of copper rings supported by tungsten bars. These masks were designed to block the soft and hard photon background coming from slit scattering off the edges of the photon collimators. The tungsten bars provided additional synchrotron protection. A schematic of the relative positions of the synchrotron collimators and the collimator masks is shown in Figure 4.10. 


\section{Chapter 5}

\section{Detectors}

The E158 detectors were mounted on a cart located at the East end of the ESA, except for the luminosity monitor which was installed further downstream, near the beam dump. All five detectors had a cylindrical geometry, symmetric with respect to the beam. Their position along the beam line is shown in Figure 5.1. The drift pipe described in the previous chapter ended right before the detector cart, at which point the beam exited the vacuum through a conically shaped flange. The first detector encountered by the signal was the profile detector, followed by a package consisting of the Møller and the ep detector, and another package containing the pion detector. A fourth detector was mounted on the side of the profile detector (not shown in Figure 5.1), which was used for polarimetry. The fifth and last detector was the luminosity monitor, positioned $\sim 7 \mathrm{~m}$ downstream of the cart. This chapter consists of a brief description of each detector.

\subsection{Profile Detector}

The purpose of the profile detector was to measure accurately the Møller and the ep flux, which, as it will be shown in Section 6.3.2, was used to determine the ep contribution to the measured parity-violating asymmetry. This detector was positioned immediately upstream of the Møller and ep detector. It consisted of four Cerenkov counters, each composed of a quartz cylinder, followed by a lightguide and a photomultiplier tube (PMT). The counters were attached to the back of a wheel (when 


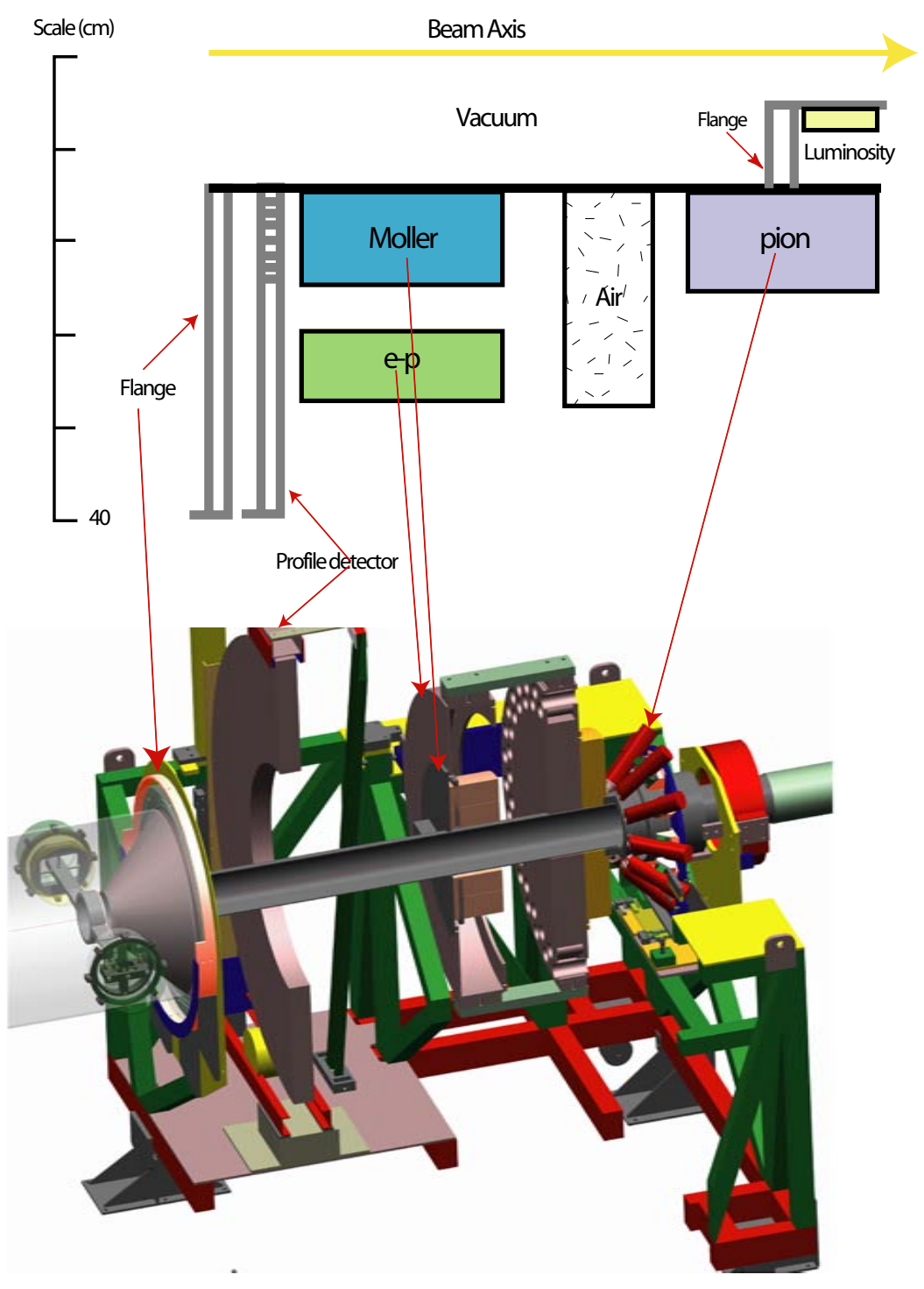

Figure 5.1: The E158 detectors. Top: schematic of the side view of the bottom half of the detector cart. Bottom: a 3-D rendering of the detector cart. All detectors had cylindrical symmetry. Note that the polarimetry detector is not shown, but was mounted on the back and to the right of the profile detector when looking downstream. 
looking downstream), which could rotate by $180^{\circ}$. The counters were mounted on position tracks, allowing them to move radially inward or outward along the plane of the wheel (Figure 5.2). The innermost position was $15 \mathrm{~cm}$. This position was the closest the counters could be to the beam without hitting the beam pipe. The outermost position was $55 \mathrm{~cm}$, so that the counters would not interfere with the Møller flux [9] during normal operation.

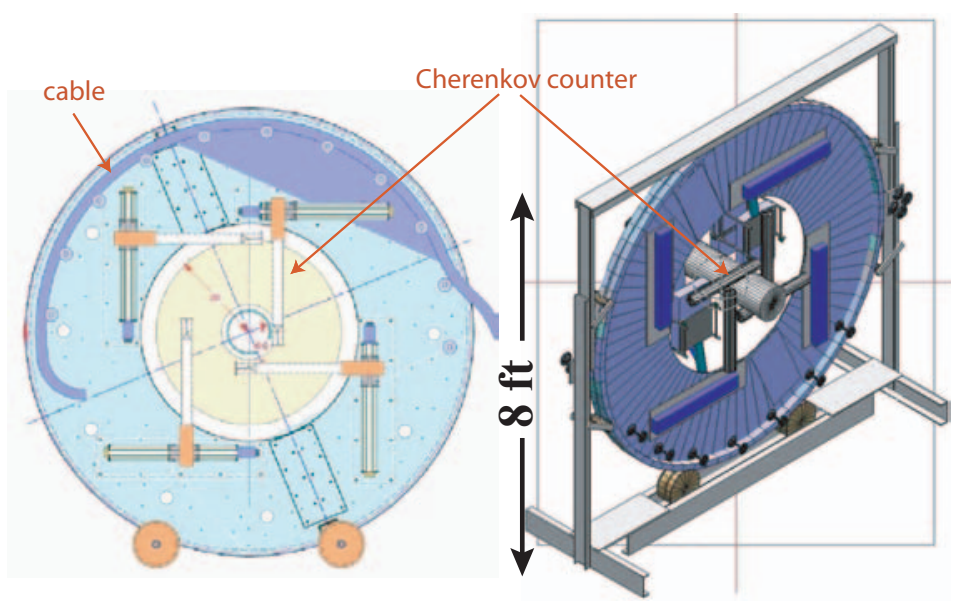

Figure 5.2: Schematic of the profile detector. One can see the four Cerenkov counters mounted to the wheel. Depicted in darker blue on the left figure is the cable used to rotate the wheel.

To increase the measuring flexibility of the detector, more components were added to two of the Cerenkov counters after the initial commissioning. First, there was the option of placing a tungsten pre-radiator in front of any of these two counters during calibration runs, which served to block low momentum particles. Second, one could also insert a shutter in front of either counter's PMT, which blocked all the photons from the quartz leaving only the signal originating from the PMT itself (Figure 5.3). The signal originating from the PMTs had to be measured, since their shielding did not prevent all particles from hitting the PMTs. The motion of each part of the profile detector (the wheel, the counters, the pre-radiators and the shutters) was controlled remotely from the Counting House by a LABVIEW program. The signals from the Cerenkov counter PMTs were connected via BNC cables to CAMAC 2219W 11-bit ADCs. 


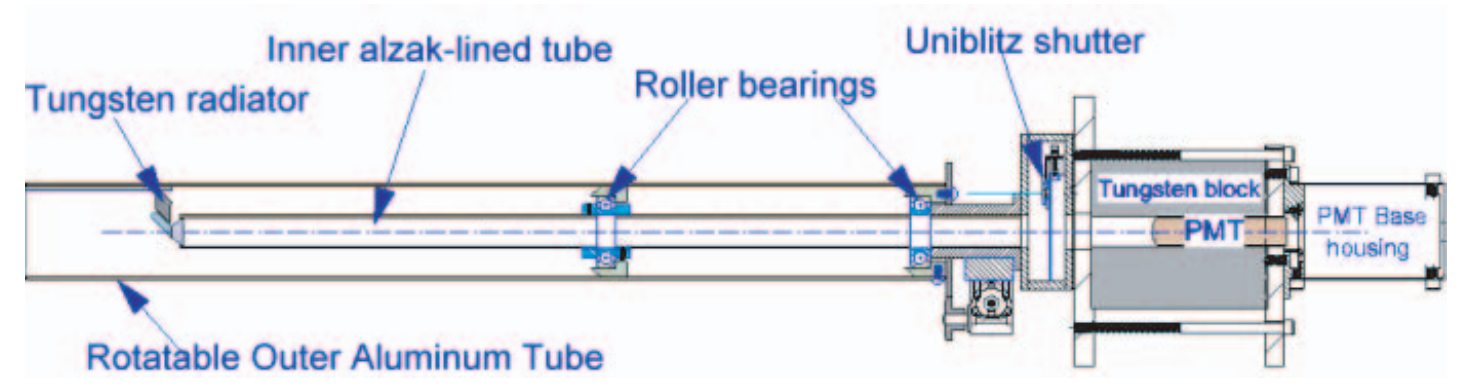

Figure 5.3: Schematic of a Cerenkov counter and the moving mechanism.

\subsection{The Møller and the ep Detector}

The Møller and the ep detector consisted of two concentric, ring-shaped assemblies, which were built in a similar fashion. The inner ring had an acceptance region sensitive to the Møller electrons, while the outer one was sensitive to the ep electrons (Figure 5.4). The detector was designed to maximize its response to electrons in the range of $10-24 \mathrm{GeV}$ and have an energy resolution of $\sigma_{E} / E \approx 10 \%$. Additionally, it had to be able to sustain high radiation doses of about 5 Mrad per week. Finally, the detector had to be azimuthally symmetric and measure flux at different radii and angles.

To satisfy all these requirements the detector design was based on the quartz fiber calorimetry technique. It consisted of layers of quartz fibers, sandwiched between slabs of copper. The copper acted as the absorber, while the fibers were the active medium. As particles showered in the absorber, they generated Cerenkov light in the quartz fibers. The produced light was then taken through mirrored lightguides to PMTs. The particular fibers used in the detector were made of amorphous silica. The light guides were created from highly polished aluminum sheets, and the PMTs were Hamamatsu R2154 model [70].

\subsubsection{Detector Geometry}

The Cerenkov radiation is emitted in a cone at an angle of $\sim 45^{\circ}$ relative to the beam direction. In order to maximize the signal picked up by the fibers, each copper-fiber- 


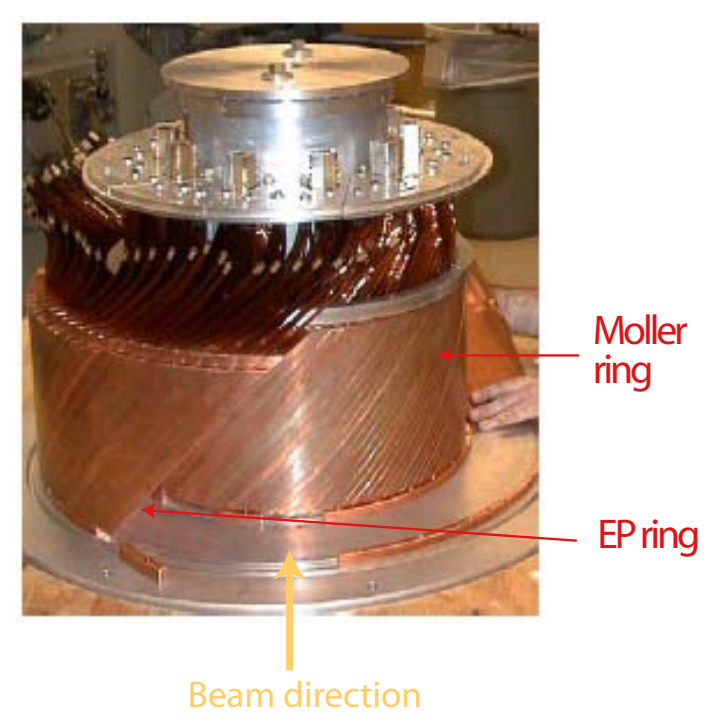

Figure 5.4: A photograph of the Møller and ep detector as the ep ring was being assembled. The lightguides would be attached to the fibers protruding out of the copper slabs.

copper wedge was oriented at a $45^{\circ}$ angle to the incident particles. Both the Møller and the ep rings consisted of 100 such wedges. The detector was 16 r.l. and 1.42 i.l. thick, long enough to keep the shower fluctuations to $<10 \%$ but short enough for the pions not to interact. The orientation of the wedges as well as the detector dimensions are depicted in Figure 5.5.

In order to provide radial and azimuthal segmentation for the Møller detector, the fibers were divided into three layers, which covered the inner, middle and outer region. Furthermore, the fibers from a particular layer originating from adjacent wedges were bundled together into a cluster which was connected to one single PMT. As a result, the Møller detector was divided into three smaller rings (inner, middle and outer), with the inner ring further divided into 10 segments and the middle and outer rings divided into 20 segments, respectively. The ep section of the detector consisted of one single ring divided into 10 segments. Every PMT corresponded to one segment, also known as a "channel". During the experiment, data was recorded from a total of 60 Møller and ep channels. A detailed description of the detector geometry and segmentation is given in Reference [70]. 


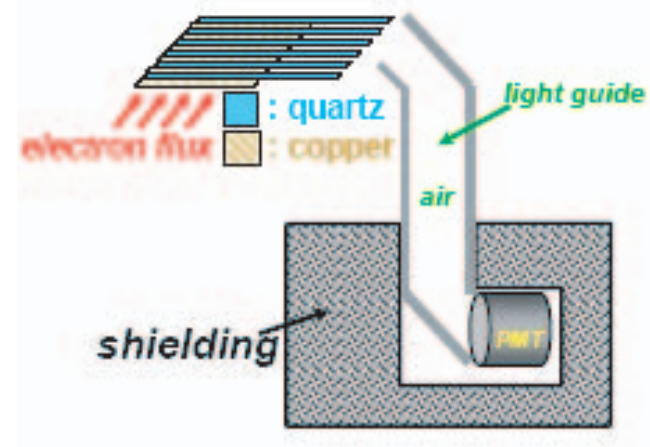

(a)

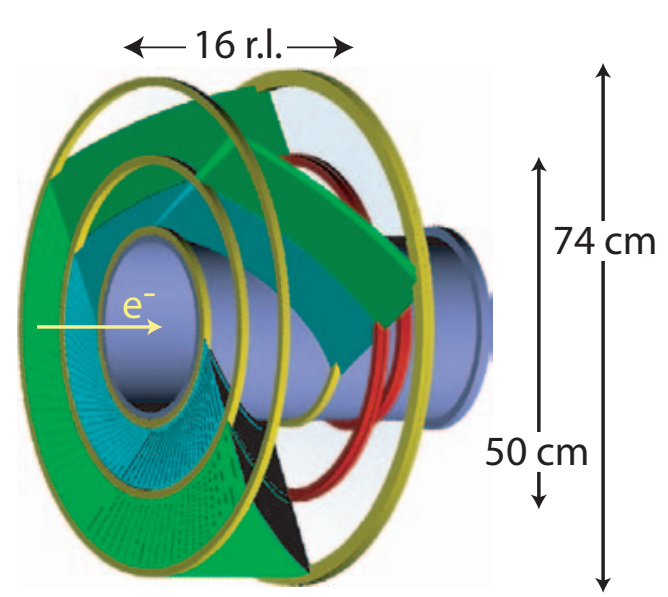

(b)

Figure 5.5: (a) Schematic of the Møller detector. Quartz fibers are sandwiched between copper slabs. (b) Schematic of the the orientation of copper slabs and fibers with respect to the incident beam. The copper-fiber-copper wedges are separate for the Møller and ep rings. Also shown are the outer diameters for each ring.

\subsubsection{Detector Electronics}

The electronics for the Møller and ep detector were divided between two locations: near the detector in ESA, and in an adjacent building $60 \mathrm{~m}$ away, known as the electronics hut. This building also contained the electronics for the other E158 detectors. The reason for the division was to perform repairs, tests and modifications of the electronics without having to access the experimental hall.

A diagram for the Møller and ep electronics is shown in Figure 5.6. The high voltage (HV) for the PMTs originated from a high voltage supply in the electronics hut. The HV RG59 cables were routed from the electronics hut to the detector PMTs. Each PMT signal passed through a low-pass filter in order to prevent reflections and to decelerate the pulse, such that all of its energy would be deposited into the isolation transformers that followed each filter. The purpose of the isolation transformers was twofold. First, they prevented ground loops between the PMTs. Second, by connecting capacitors and resistors to the isolation transformers, together they acted as a ringing RLC circuit, similar to the one for toroids described in Section 3.1.

Once the PMT signals were transformed into ringing signals, they were amplified 
by a differential pre-amplifier, which offered two gain stages: one stage of $1 / 2 / 4 / 8$ gains and one stage of 10/100 gains. During the data collection the gains of 1 and 100 were used, since they maximized the signal resolution. After amplification, the PMT signals were taken from ESA to the electronics hut via twisted pair cables, in order to prevent cross-talk. Inside the hut, they were rectified by means of "absolute value" circuits, in a similar fashion to the toroid signals. Finally, the PMT signals were fed to ADCs identical to the ones used for the toroids.

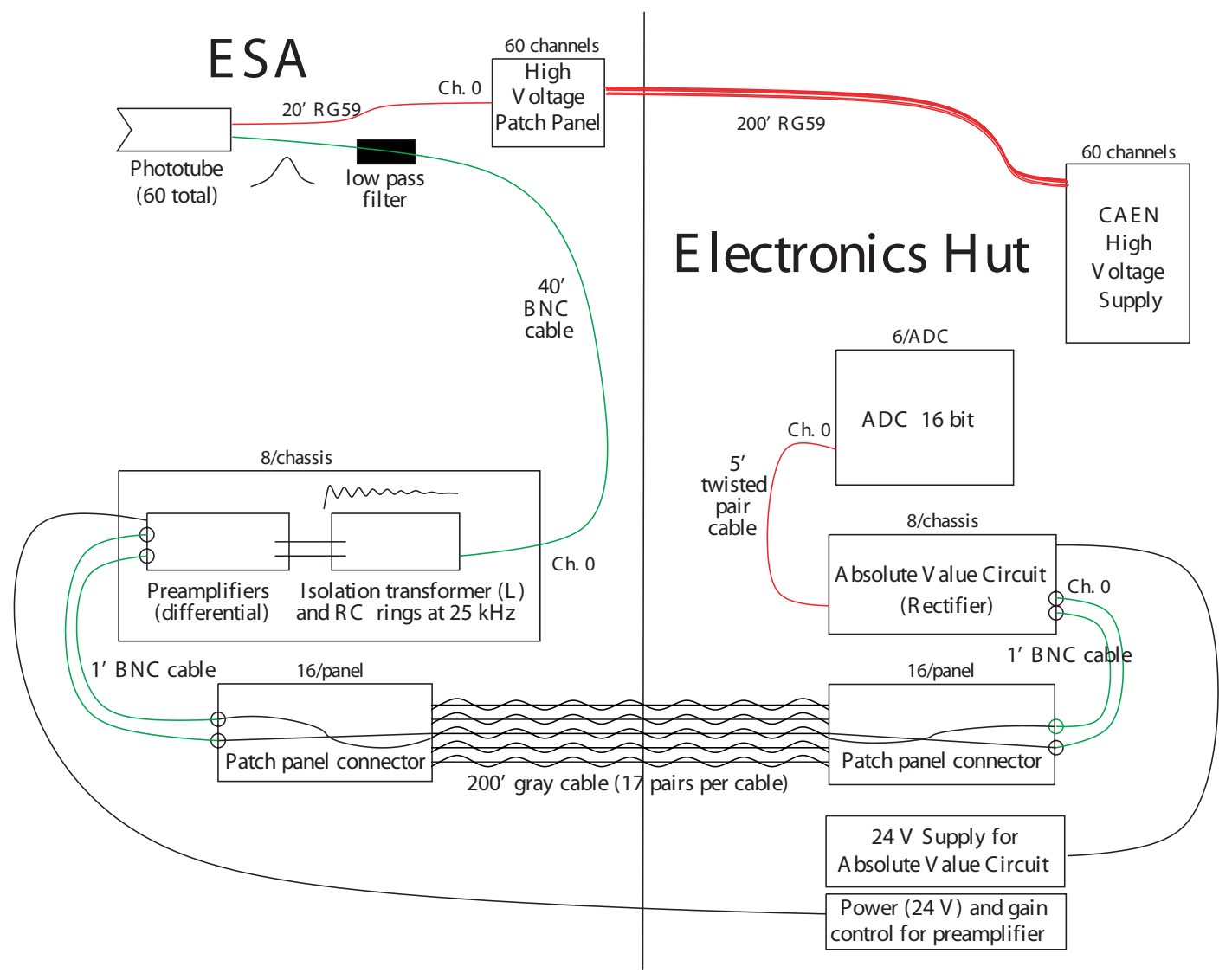

Figure 5.6: Schematic of the Møller and ep detector electronics.

If the signal contribution to the width of the experimental asymmetry distribution were zero, then only the noise due to electronics would be contributing to this width. Such a scenario would be possible if there were infinite statistics. Since this scenario is impossible, the electronics contribution (i.e., electronics resolution) was determined by running the beam at four different current settings and calculating the width of 
the experimental asymmetry for each setting. Figure 5.7 shows the linear fit to a plot of the square of the experimental asymmetry width versus the inverse beam current, $1 / \mathrm{N}$, where $\mathrm{N}$ is the number of electrons. The electronics resolution is given by the offset, at which point $\mathrm{N}=\infty$ and the resolution is $110 \mathrm{ppm}$. This amount of electronics contribution to the experimental asymmetry width is non-negligible, especially since the width of the Møller detector asymmetry during the experiment was 190-220 ppm. Bench measurements of the Møller electronics indicated that the most likely causes for the $110 \mathrm{ppm}$ electronics resolution were pre-amplifier and pedestal noise, as well as electronics cross-talk.

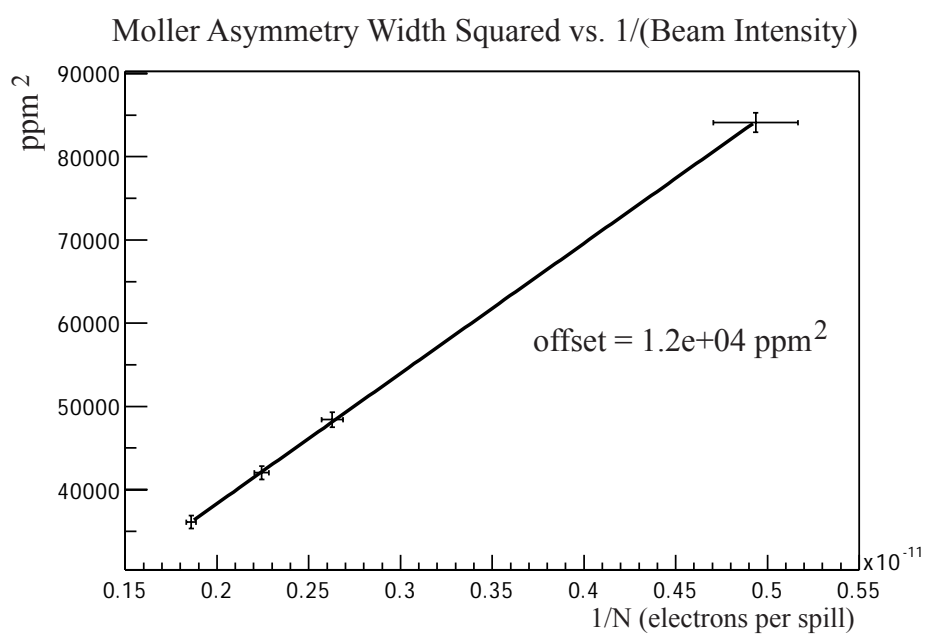

Figure 5.7: Plot of the square of the experimental asymmetry width $\left(\sigma_{\text {exp }}^{2}\right)$ versus the inverse of beam intensity $(1 / \mathrm{N}, \mathrm{N}=$ number of electrons.) The Møller detector resolution is given by the square root of the offset: $\sqrt{12000 \mathrm{ppm}^{2}}=110 \mathrm{ppm}$.

\subsection{Pion Detector}

The pion detector measured the pion flux and asymmetry in the acceptance region of the Møller and ep detector. It consisted of 10 fused quartz cylinders, $10 \mathrm{~cm}$ long and $4 \mathrm{~cm}$ in diameter, mounted around the beam pipe, with Phillips XP2232B PMTs attached to each end (Figure 5.8). The cylinders were tilted by $45^{\circ}$ with respect to the beam direction in order to maximize Cerenkov light signal, similar to the quartz 
fibers in the Møller detector. The pion detector covered a disk-shaped area behind the Møller detector of 30 and $47 \mathrm{~cm}$ in inner and outer diameter, respectively [39].

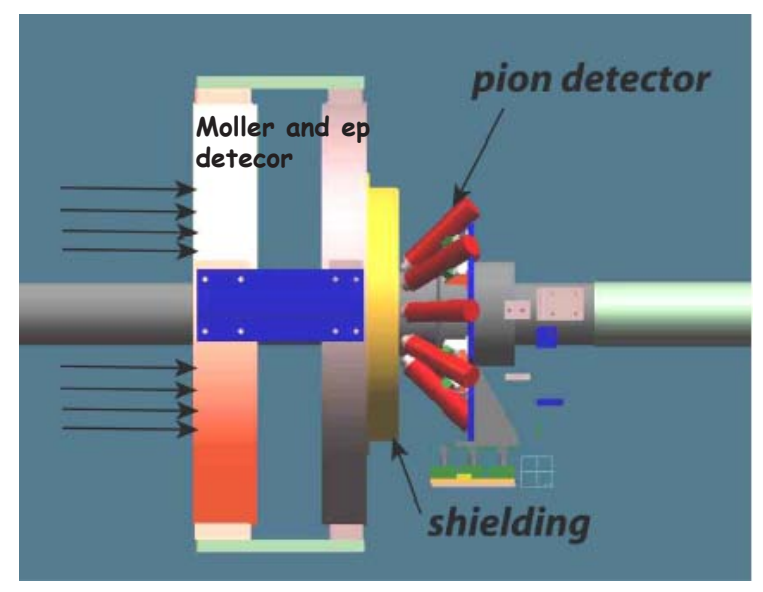

Figure 5.8: Schematic of the Pion detector (in red) sitting behind shielding and the Møller and ep detector.

To reduce backgrounds, $25 \mathrm{~cm}$ of lead shielding were inserted between the pion and the Møller detector, including some extra shielding around the beam pipe. Taken together, the Møller detector and the lead shielding amounted to 60 r.l. in front of the pion detector. This thickness was adequate to block out most of the Møller electron flux from punch-through, which was initially hundreds of times larger than the pion flux. From simulations, the energy resolution of the pion detector was estimated to be $\sigma_{E} / E=150 \%$ ( $E$ is the mean energy of the pion distribution), and the signal fluctuations to be of the order of $0.1 \%$. The pion PMT signals were taken via BNC cables directly to the electronics hut, where they were plugged into ADCs similar to the ADCs used for BPMs, described in Section 3.2.3.

\subsection{Polarimeter Detector}

The polarimeter detector was used to determine the longitudinal polarization of the electron beam by measuring the scattering cross section asymmetry produced when the beam hits the polarized supermendur foils described in Section 4.1.1. The asym- 
metry $A_{\text {polarization }}$ is given by

$$
A_{\text {polarization }}=P_{z}^{\text {beam }} \times P_{z}^{\text {target }} \times \frac{\left(7+\cos ^{2} \theta_{C M}\right) \sin ^{2} \theta_{C M}}{\left(3+\cos ^{2} \theta_{C M}\right)^{2}},
$$

where $P_{z}^{\text {beam }}$ is the beam's longitudinal polarization, $P_{z}^{\text {target }}$ is the longitudinal polarization of the foil electrons ( $\sim 8 \%$ for the experiment) and $\theta_{C M}$ is the scattering angle in the center of mass frame.

During the polarization measurement, the hydrogen target was removed and the foil target was moved into the beam. Moreover, to reduce the ep background, the "holey" collimator (see Section 4.2.4) was inserted, and the spectrometer quadrupole strengths were set to different values compared to the normal running conditions. The measurement lasted typically $\sim 10-15$ thousand pulses (spills), and was repeated throughout the experiment after every half-wave plate reversal, which happened approximately every other day.

The polarimeter was a Cerenkov calorimeter, consisting of six quartz plates sandwiched between seven tungsten plates. The first two plates at the face of the detector were tungsten followed by alternating quartz and tungsten plates. The calorimeter was attached to a horizontal lightguide made of an aluminum tube with a reflecting foil inside. The light from the guide was reflected off a mirror, such that it hit a Hamamatsu R2154-02 PMT (Figure 5.9). To increase the light collection efficiency, the tungsten plates had a single side coated with a reflecting foil, and all the plates were tilted by $30^{\circ}$. The entire polarimeter assembly was shielded by $15 \mathrm{~cm}$ of lead [11].

The Cerenkov calorimeter unit could move vertically in and out of the Møller scattering region, so that it was not present during normal data collection. The vertical movement was controlled remotely from the Counting House. A BNC cable connected the polarimeter detector PMT to a CAMAC 2219W 11-bit ADC in the electronics hut. 


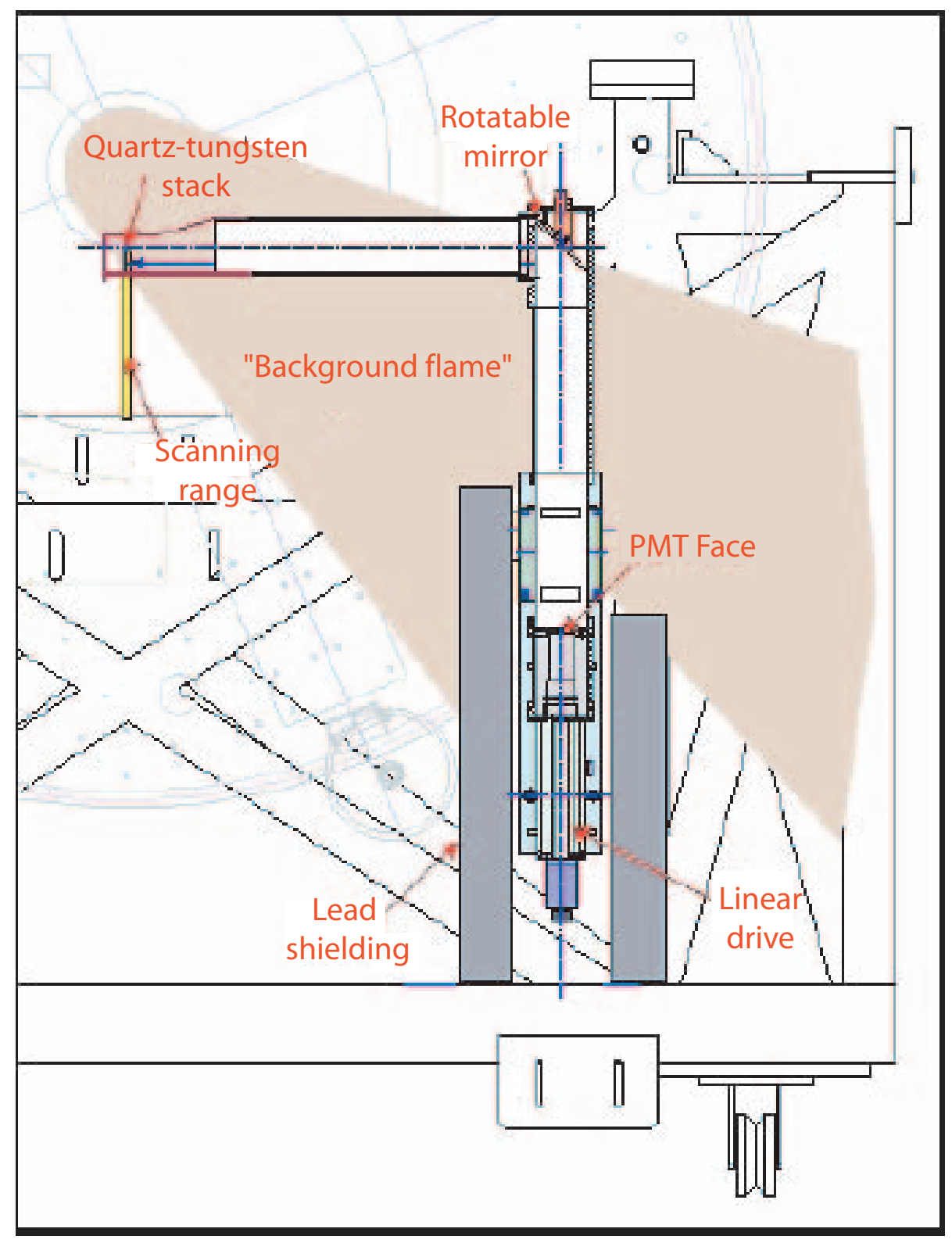

Figure 5.9: Schematic of the polarimeter detector. 


\subsection{Luminosity Monitor}

The luminosity monitor was the final detector in the detector package and was located in the Beam Dump East, just outside the ESA. Its purpose was twofold. First, it measured the asymmetry of very forward angle Møller and ep scattered electrons. Since the physics asymmetry for these low angle scatters is very small ( $\sim 10 \mathrm{ppb})$, the asymmetry measured by the luminosity monitor should be null, within its precision. The luminosity monitor served to check for false asymmetries. Second, the luminosity monitor was used to keep track of target density fluctuations, by looking at the ratio of the observed signal to the beam intensity and the correlation of the Møller detector asymmetry to the luminosity monitor asymmetry.

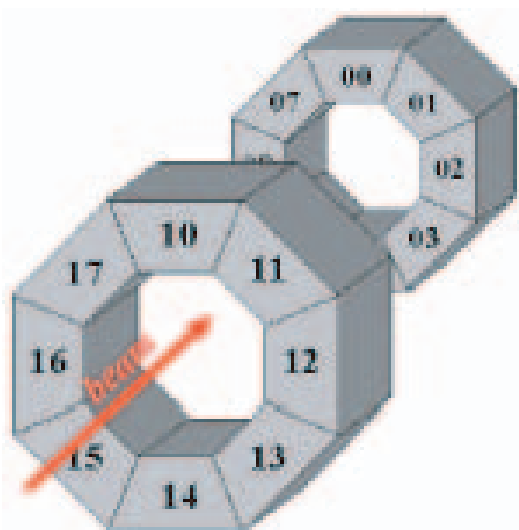

(a)

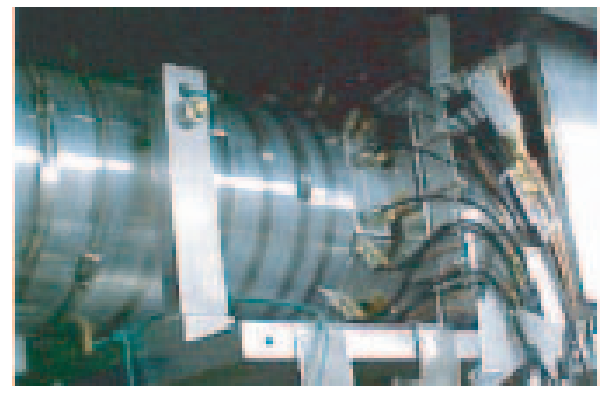

(b)

Figure 5.10: (a) Diagram of the luminosity monitor chambers. (b) Photograph of the luminosity monitor.

The flux hitting the luminosity monitor was very large, compared to the other detectors. To sustain linearity in the face of such flux, the luminosity monitor was designed as an ion chamber type detector. It consisted of two separate rings, concentric to the beam of $7 \mathrm{~cm}$ and $10 \mathrm{~cm}$ in inner and outer diameter, respectively. There were 7 r.l. of aluminum installed in front of the first ring and 4 r.l. of aluminum in front of the second ring. The aluminum served as a pre-radiator and as a shield for blocking synchrotron radiation. Each ring was divided into eight identical chambers (one for each octant), as shown in Figure 5.10. Every chamber was filled with nitro- 
gen and contained a set of eleven parallel plates with alternating plates held at 0 or $100 \mathrm{~V}$. As charged particles traversed the chamber they generated ions, which were collected on the plates [33]. The signals from each chamber passed through Foam 8 BNC cables into ADCs similar to the BPM ADCs, located in the electronics hut.

\subsection{Good Spill and Bad Spill Monitors}

In addition to the five detectors described above, there were two ion chambers located under the beam pipe $\sim 2 \mathrm{~m}$ upstream and $\sim 0.5 \mathrm{~m}$ downstream of the target. The downstream ion chamber, known as the good spill monitor, measured wide angle scattered flux from the target. The upstream one, known as the bad spill monitor, measured the amount of beam halo particles. The signals from both ion chambers were taken via BNC cables to the Counting House, where they were split in two pieces. One part was plugged into a CAMAC 2219W 11-bit ADC, while the other

part was plugged into an oscilloscope. During the data collection, the oscilloscope traces of the good and bad spill monitors were used to tune the accelerator, such that the beam halo would be minimized. The reason for minimizing the beam halo was to prevent scatters upstream of the target from entering the spectrometer. 


\section{Chapter 6}

\section{Analysis and Results}

The goal of the analysis was to extract the physics asymmetry from the raw asymmetry, namely, the asymmetry obtained before any corrections were applied to the data. The analysis needed to determine and remove contributions due to beam helicity correlations, random fluctuations and parity-violating backgrounds. Moreover, the normalization factors related to the beam polarization and the dilution factors generated by backgrounds had to be taken into account. This chapter describes the methods used to obtain the physics asymmetry and reports the results from Run I and Run II. The analysis for Run III is underway.

\subsection{The Møller Detector Analysis}

\subsubsection{Calculating the Raw Asymmetry}

As described in Section 5.2, the Møller detector consisted of 50 channels (the other 10 belonged to the ep detector). The signal from every channel was recorded on a pulse by pulse basis. The first step towards determining the raw asymmetry for each channel was to calculate and subtract the pedestals from the raw signals. This process was done in two passes through the data. In the first pass a running average of pedestals was calculated for every channel in each timeslot (defined in Section 2.2.1). Averaging every ten pedestals was sufficient to determine the pedestal noise. The pedestal subtraction took place during the second pass. 
Once the pedestals were removed, the raw asymmetry per pulse pair of channel $i$ was calculated as

$$
A_{i}^{\text {raw }}=\frac{\frac{S_{i R}}{Q_{R}}-\frac{S_{i L}}{Q_{L}}}{\frac{S_{i R}}{Q_{R}}+\frac{S_{i L}}{Q_{L}}},
$$

where $S_{i R}$ and $S_{i L}$ are the channel signal sizes for a right- and left-handed helicity pulse, respectively. Since the signal size was proportional to the beam charge, the signals were normalized by $Q_{R}$ and $Q_{L}$, corresponding to the charge measurement for a right- and left-handed helicity pulse, respectively. As a result, the dependence of the asymmetry on charge fluctuations was removed.

\subsubsection{Removing Beam Helicity Correlations}

The raw asymmetry $A_{i}^{\text {raw }}$ had to be corrected for a residual asymmetry in the beam trajectory, which affected both the central value of the raw asymmetry and its width. The electron beam trajectory could be described by six parameters: charge, energy, $\mathrm{x}$ and y angle, and $\mathrm{x}$ and y position. The helicity correlations (i.e., the asymmetries) of these parameters were measured by toroids or BPMs. To remove the contributions to the raw asymmetry arising from beam helicity correlations, two independent methods were used: regression and dithering.

\subsubsection{Regression}

The correlation between beam and channel raw asymmetries was linear to first order, as shown in Figure 6.1 (c). Consequently, it was possible to remove the beam contributions by applying a least squares linear regression to the raw asymmetry results for each channel. This was achieved by plotting multi-dimensionally all the channel asymmetries versus the six beam parameters. By applying a single linear fit to the data, the regression slopes $m_{i j}$ were obtained for every channel $i$ and beam parameter $j$. The regressed channel asymmetry $A_{i}^{\text {regressed }}$ is then given by

$$
A_{i}^{\text {regressed }}=A_{i}^{r a w}-\sum_{j} m_{i j} b_{j}
$$


where $b_{j}$ is the asymmetry for beam parameter $j$ and $m_{i j}=\frac{\partial A_{i}^{\text {raw }}}{\partial b_{j}}$.

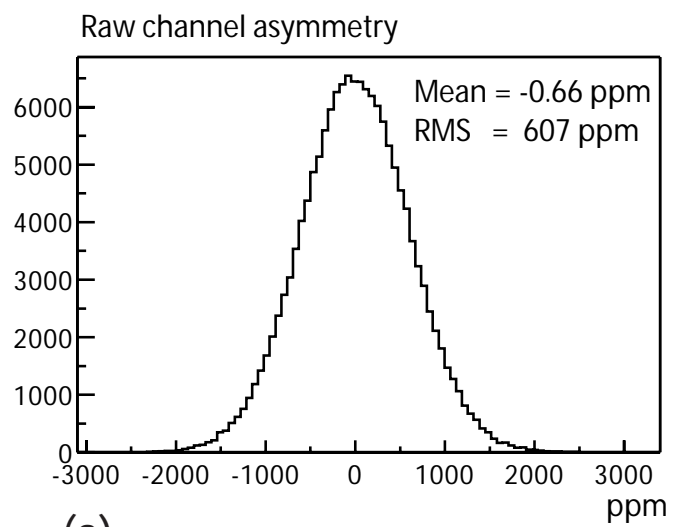

(a)

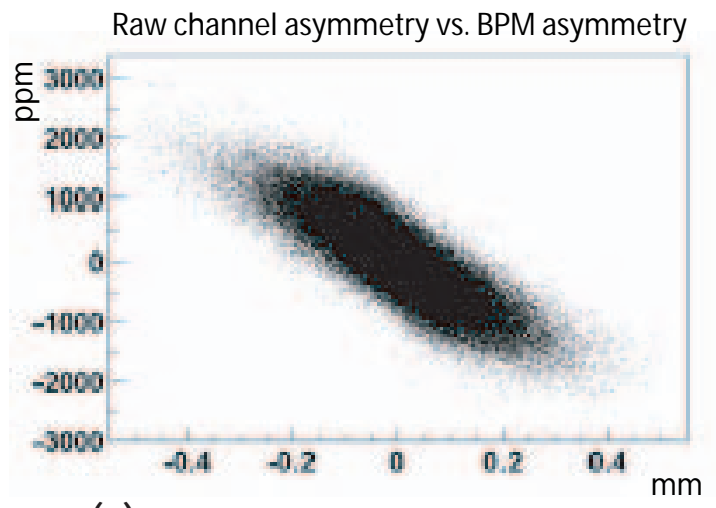

(c)
Regressed channel asymmetry

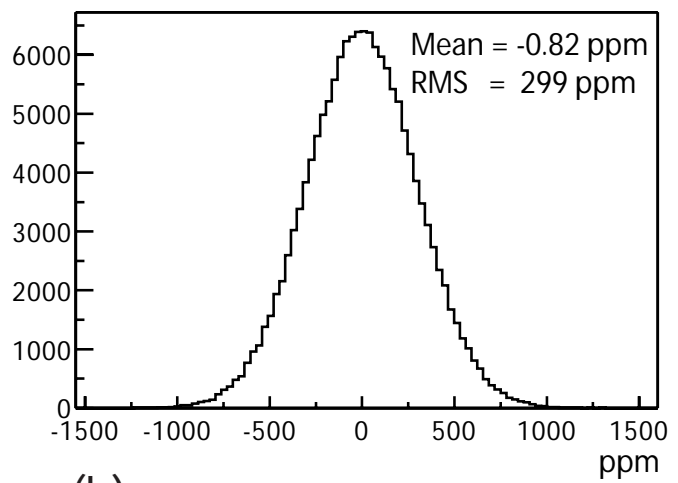

(b)

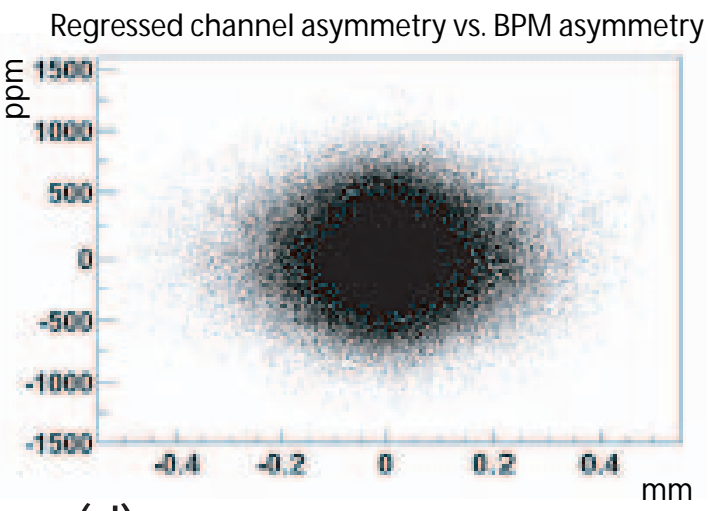

(d)

Figure 6.1: (a) The raw asymmetry of a single channel taken over one hundred thousand pulse pairs, namely, two hundred thousand pulses. (b) The regressed asymmetry of the same channel. Note how the RMS has been reduced by more than a factor of two. (c) Raw channel asymmetry versus position asymmetry. (d) Same plot as in (c), except that the channel asymmetry is now regressed out. The first-order position dependence has been removed.

Since the regression slopes $m_{i j}$ varied over time, they were calculated for every ten thousand pairs in each timeslot, which was enough to minimize the statistical uncertainties of the fit. For the regression to work, the beam jitter for each beam monitor had to be small compared to the slopes, a requirement which was satisfied during the experiment by maintaining the average jitter at the level of 20-100 $\mu \mathrm{m}$. Given that each beam parameter was measured by at least two independent monitors, the regression was performed against the average. One drawback of the regression 
method was that it favored the quieter monitors, being that the regression slopes for those monitors generally had the smallest uncertainties. However, the monitor with the smallest noise was not necessarily the one most sensitive to beam fluctuations. Given that regression was monitor-dependent, another method of determining the correlation between the beam and channel raw asymmetries was needed as a crosscheck.

\subsubsection{Dithering}

The difference between the dithering and regression methods was rooted in the way that the correlation slopes $m_{i j}$ were calculated. The dithering correlation slopes were calculated over portions of the data during which all six beam parameters were dithered about their nominal value by an amount large compared to the beam jitter. The beam dithering was achieved with the help of eight corrector magnets. If $C_{k}$ is the strength of a corrector magnet $k$, then [20]

$$
\frac{\partial A_{i}^{\text {raw }}}{\partial C_{k}}=\sum_{j} \frac{\partial A_{i}^{r a w}}{\partial b_{j}} \frac{\partial b_{j}}{\partial C_{k}}=\sum_{j} m_{i j} \frac{\partial b_{j}}{\partial C^{k}} .
$$

Here $\frac{\partial b_{j}}{\partial C^{k}}$ is the correlation between the asymmetry of beam parameter $j$ and the strength of corrector magnet $k$. Since channel and beam asymmetries, as well as the strengths of corrector magnets were known, the slopes $m_{i j}$ were obtained by performing a $\chi^{2}$ minimization to Eq. 6.3 with respect to $m_{i j}$. Similar to regression, the corrected asymmetry per channel is given by

$$
A_{i}^{\text {dithered }}=A_{i}^{\text {raw }}-\sum_{j} m_{i j} b_{j}
$$

During the experiment the beam was dithered periodically with a $\sim 4 \%$ duty cycle. The data obtained during dithering cycles was only used to determine the dithering slopes and was not used in the data analysis that determined the physics asymmetry. Compared to regression, the dithering method had the advantage that it did not depend on the monitor resolutions. However, during the beginning of Run I, beam 
dithering was not very reliable due to hardware problems with the coil magnets. Consequently, the method utilized to produce the final result was regression, with dithering serving as a cross-check.

\subsubsection{Calculating the Overall Asymmetry}

The most straightforward way to calculate the overall parity-violating asymmetry for the Møller detector would have been to add all the channel asymmetries. However, this simple summation ignored statistical variations among different channels, each of which detected a different particle flux. As a result, the individual channel asymmetries had to be weighted by their width $\sigma_{i}$, calculated on a per-run basis ${ }^{1}$. The detector asymmetry per run in this case is

$$
\begin{gathered}
w_{i}=\frac{1}{\sigma_{i}^{2}} \\
A_{\text {detector }}^{\text {run }}=\frac{\sum_{i=1}^{50} A_{i} w_{i}}{\sum_{i=1}^{50} w_{i}},
\end{gathered}
$$

where the summation is over only 50 channels out of 60 , since the 10 channels belonging to the ep detector are not included.

The weighting scheme represented in Eq. 6.6 was improved even further to account for common mode electronic noise among the channels and for different noise levels in each channel. From the covariance matrix of the detector

$$
M_{i j}=M_{j i}=\frac{1}{N_{\text {pulses }}}\left(\sum_{\text {pulses }} A_{i} A_{j}\right)-\frac{1}{N_{\text {pulses }}^{2}}\left(\sum_{\text {pulses }} A_{i} \sum_{\text {pulses }} A_{j}\right),
$$

the weights $w_{i}$ were calculated by minimizing

$$
\min \left[\sum_{i, j} w_{i} w_{j} M_{i j}\right] \Longrightarrow w_{i}
$$

\footnotetext{
${ }^{1}$ During the experiment the data was taken over series of runs which lasted $\sim 400,000$ pulses each. These runs are not to be confused with Run I and Run II, which include the overall data taking period during spring and fall 2002, respectively.
} 
Combining the correction slopes described in the previous section with the channel weights, the overall detector asymmetry per run is given by

$$
A_{\text {detector }}^{\text {run }}=\frac{1}{N_{\text {pulses }}} \sum_{\text {pulses }} \frac{\sum_{i} A_{i, \text { pulse }}^{\text {raw }}-\sum_{i, j} m_{i j}^{\text {pulse }} b_{j}^{\text {pulse }} w_{i}}{\sum_{i} w_{i}} .
$$

To calculate the asymmetry over several runs $\left(A_{\text {total }}\right)$, the asymmetry per run $\left(A_{\text {detector }}^{\text {run }}\right)$ was weighted by the width in the asymmetry distribution for that run $\left(\sigma_{\text {run }}\right)$ :

$$
\begin{gathered}
w_{\text {run }}=\frac{1}{\sigma_{\text {run }}^{2}} \\
A_{\text {total }}=\frac{\sum_{\text {run }} A_{\text {detector }}^{\text {run }} w_{\text {run }}}{\sum_{\text {run }} w_{\text {run }}} .
\end{gathered}
$$

\subsubsection{Blind Analysis}

The predicted physics asymmetry is so small that extra care had to be taken in order to prevent human bias during the analysis. As a result the data was "blinded", following an algorithm in which the raw asymmetry of each Møller channel was shifted by a fixed amount and then multiplied by a factor of 1 or -1 :

$$
A_{i, \text { blinded }}^{\text {raw }}= \pm\left(A_{i}^{\text {raw }}+A^{\prime}\right)
$$

where $A^{\prime}$ was the introduced shift. The theoretical prediction for the asymmetry is $\sim-150 \mathrm{ppm}$, so $A^{\prime}$ was chosen to fall in the interval between -200 to +200

ppm. All the cuts and systematic studies were performed on the "blinded" data. Once the analysis was completed, the data was "unblinded" revealing the real Møller asymmetry. It should be pointed out that the data from the other detectors was not blinded, only the parity-violating asymmetry was blinded.

\subsubsection{Analysis Data Selection}

There were two kinds of analysis cuts applied to the E158 data. The first kind, also known as baseline cuts, included all cuts made before the data had undergone any 
detailed analysis. Baseline cuts removed all data taken during hardware failures or other conditions which rendered the data suspect. The second kind of cuts were applied to reduce systematic uncertainties. All cuts had to remove as few pulses as possible and avoid introducing any bias to the data. This section gives a list and a brief description of analysis cuts; a detailed description is given in Reference [43].

\subsubsection{Baseline Cuts}

There were a total of nine baseline cuts:

1. Beam level cut, which removed pulses for which the toroids measured less than $1 \cdot 10^{11}$ or more than $7 \cdot 10^{11}$ electrons per pulse.

2. Pedestal cut, which removed pedestal pulses.

3. Timeslot cut, which ensured that both members of a pulse pair belonged to the same timeslot, thus removing $60 \mathrm{~Hz}$ noise.

4. DiffTrigger cut, which was a variable that measured the time elapsed between a pulse and the preceding one. Both members of a pulse pair had to have the same diffTrigger. This cut was applied due to the fact that toroid gains were weakly influenced by the time between the arrival of two consecutive pulses. Therefore if diffTrigger was different for the pulses in a pair, this pair would have a false large asymmetry.

5. Dithering cut, which removed the data taken during beam dithering.

6. "Mixed-up spill" cut. There were times during the experiment when some loss of synchronization occurred among various ADC readings. Such scrambled pulses were called "mixed-up spills" and were removed from the data.

7. Voltage cut, which accepted only pulses for which CP and PS Pockels cell voltages (described in Section 2.1.3) were within $\pm 2000 \mathrm{~V}$, making sure that the beam feedback operated correctly. 
8. Møller ADC cut, which prevented the use of saturated, or broken ADC channels.

9. Unphysical asymmetry cut, which removed any Møller channel which recorded a raw asymmetry above 0.9 . This would happen as a result of the DAQ failing to properly read an ADC board.

\subsubsection{Reducing Systematic Effects}

As it was already mentioned, analysis cuts were applied to the data with the purpose of reducing systematic effects. In order to avoid bias, these cuts were "stretched" in time by additionally removing a few hundred pulses before and after the pulses suspect of introducing systematic uncertainties. There were eight cuts to control systematic effects, listed below:

1. Regression slopes cut, which eliminated the data for which there were too few pairs $(<100)$ to calculate meaningful regression slopes.

2. Beam cut, which removed pulses with large jitter, or large beam centroid excursions.

3. Toroid agreement cut, which ensured that the asymmetries measured by the four ESA toroids agreed to within 100 ppm.

4. Transmission cut, which required that ESA toroids measured at least $90 \%$ of the charge measured by the ASSET toroids, ensuring that transmission losses in the accelerator were less than 10\%. A bad transimission could introduce additional helicity-correlated asymmetries in the final measurement.

5. Energy cut, which removed "klystron cycles". Periodically during the experiment one of the accelerator klystrons would turn off and another klystron would turn on. Even though the beam energy would eventually stabilize to the desired value, during these "klystron cycles", the energy would change very rapidly causing the energy regression slopes to be meaningless. 
6. BPM phases cut, which excluded pulses with large BPM phase variations. As explained in Section 3.2.3, phase variations affect the accuracy of the position measurement.

7. Linear BPM cut, which ensured that the beam was within the region where BPMs were better than $99 \%$ linear in their response.

8. Rate cut, which accepted only the data taken at 120 or $60 \mathrm{~Hz}$ pulse rate.

Table 6.1 gives a summary of the cumulative acceptance levels of each cut for both Run I and Run II. The baseline cut removed most of the data, with the rest of the cuts removing only $\sim 7 \%$. The baseline cut for Run II eliminated less data than for Run I, mainly because there were less hardware failures such as mixed-up spills.

\begin{tabular}{|l|c|c||c|c|}
\hline \multirow{2}{*}{ Cut } & \multicolumn{2}{|c||}{ Run I } & \multicolumn{2}{c|}{ Run II } \\
\cline { 2 - 5 } & $\begin{array}{c}\text { Pulse pairs } \\
\text { (Millions) }\end{array}$ & $\begin{array}{c}\text { Acceptance } \\
\text { Percentage }\end{array}$ & $\begin{array}{c}\text { Pulse pairs } \\
\text { (Millions) }\end{array}$ & $\begin{array}{c}\text { Acceptance } \\
\text { Percentage }\end{array}$ \\
\hline None & 107.80 & $100 \%$ & 117.77 & $100 \%$ \\
\hline Baseline & 93.06 & $86.32 \%$ & 108.65 & $92.26 \%$ \\
\hline Previous + Reg. Slopes & 93.05 & $86.32 \%$ & 108.06 & $91.75 \%$ \\
\hline Previous + Beam & 89.21 & $82.75 \%$ & 103.58 & $87.95 \%$ \\
\hline Previous + Tor. Agreement & 89.21 & $82.75 \%$ & 103.58 & $87.95 \%$ \\
\hline Previous + Transmission & 89.14 & $82.69 \%$ & 103.56 & $87.93 \%$ \\
\hline Previous + Energy & 88.19 & $81.81 \%$ & 102.65 & $87.16 \%$ \\
\hline Previous + BPM phases & 86.76 & $80.48 \%$ & 101.07 & $85.82 \%$ \\
\hline Previous + BPM Linearity & 86.48 & $80.21 \%$ & 100.20 & $85.08 \%$ \\
\hline Previous + rate & 85.86 & $79.65 \%$ & 100.20 & $85.08 \%$ \\
\hline
\end{tabular}

Table 6.1: List of systematic cuts and their cumulative acceptance for both Run I and Run II.

\subsection{The Møller Detector Asymmetry}

Several methods were used to plot the Møller detector data in order to study possible systematic effects. The first one was to look at the asymmetry distribution on a run by run basis. Since each run took approximately one hour, this method looked for systematic effects on hour-long timescales. Additionally, runs were grouped into 
"slugs", where members of the same slug had the same source and energy configuration (half-wave plate setting, Asymmetry Inverter setting, 45 versus $48 \mathrm{GeV}$ beam energy). Since the source configuration was changed approximately every other day, a plot of the asymmetry versus slug covered two-day long timescales. Figure 6.2 shows plots of the Møller asymmetry versus run and versus slug for the Run II data. The average asymmetry is obtained from both plots by fitting a flat line. The fact that both averages agree and that the $\chi^{2}$ are reasonable is an indication that the results are stable over these timescales.
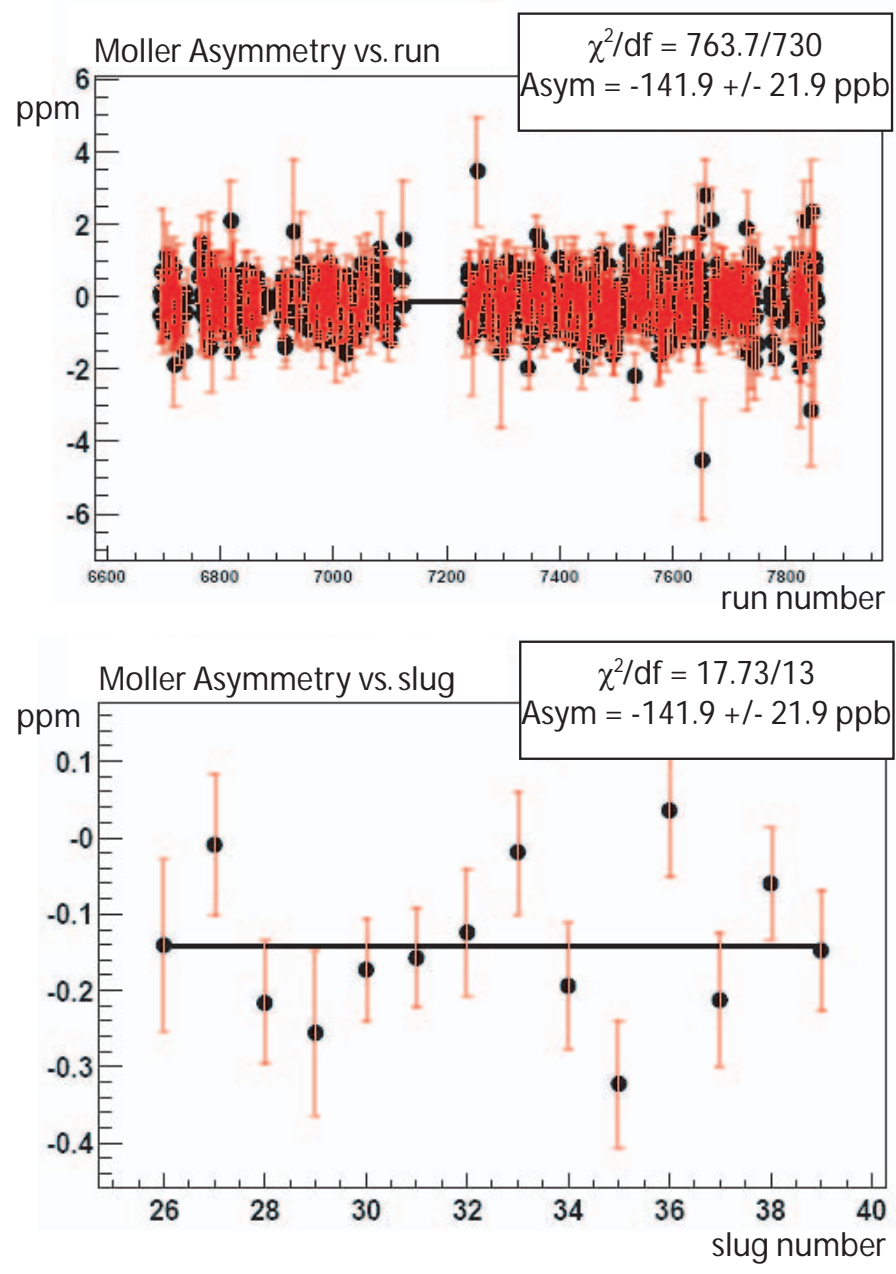

Figure 6.2: Møller asymmetry versus run and versus slug during Run II. The average asymmetries are obtained from fitting a zeroth degree polynomial to each plot.

Another powerful systematic test is to study the overall physics asymmetry av- 
erages versus reversals. As mentioned in the previous chapters, asymmetry reversals were introduced during the experiment by inserting a half-wave plate at the source and by running at 45 and $48 \mathrm{GeV}$. This led to four different sign-flip combinations: $45 \mathrm{GeV} /$ half-wave plate in, $45 \mathrm{GeV} /$ half-wave plate out, $48 \mathrm{GeV} /$ half-wave plate in, and $48 \mathrm{GeV} /$ half-wave plate out. The sign of the systematic contributions was not affected by the asymmetry reversals. Consequently, when the average asymmetry was calculated over the entire data set, the magnitude of systematic contributions was suppressed. Figure 6.3 shows the asymmetries for all four sign-flip combinations and the overall average, for both Run I and Run II.

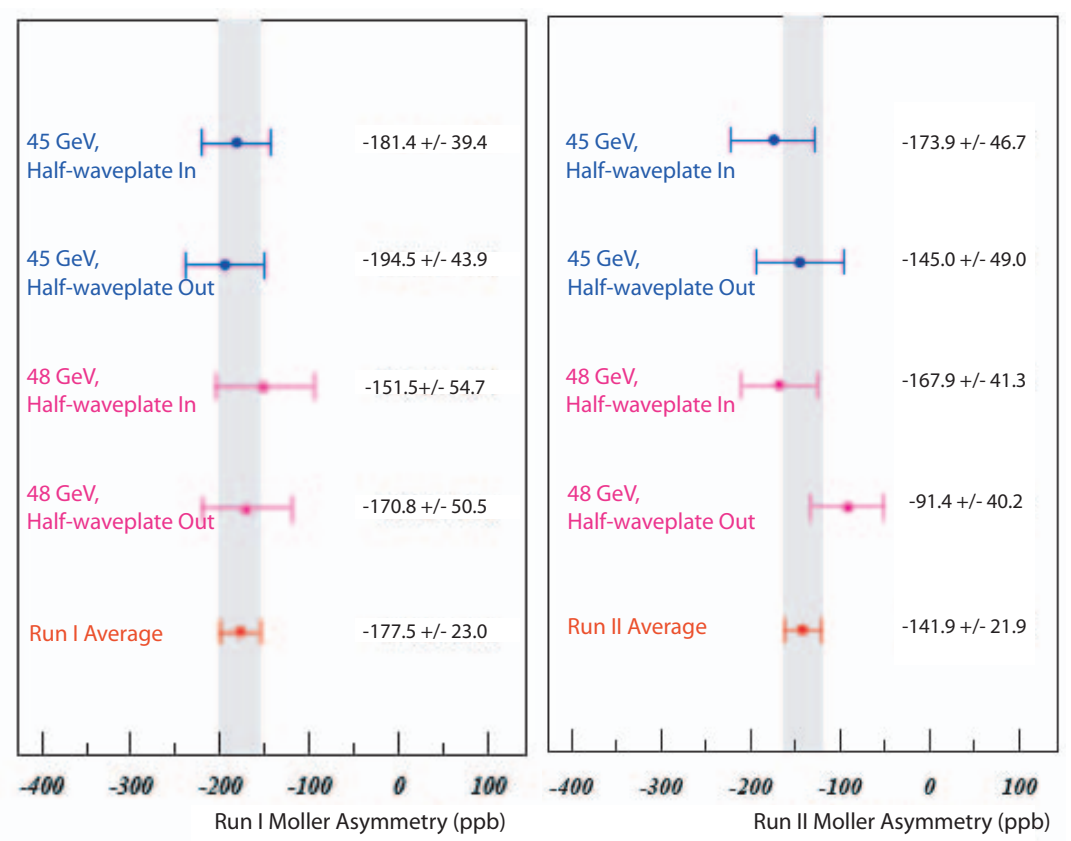

Figure 6.3: Plot of the Møller detector asymmetry for the four different sign-flip combinations and the overall average for both Run I and Run II. The asymmetries have been corrected, such that they all have the same sign.

Although not illustrated here, additional tests for systematic effects were performed on the data, such as comparing the results for each timeslot, and the asymmetries obtained from each ring. The results from these tests were consistent with the ones described above. Only the inner and middle ring of the Møller detector were used to calculate the overall Møller detector asymmetry. The outer ring turned out 
to be sensitive to higher-order moments in the beam distribution, and was used for systematic studies, discussed in Section 6.3.1.

An important test of the overall analysis was to compare the Møller asymmetry obtained by regression with that obtained by dithering. Both asymmetries were found to be within $5 \mathrm{ppb}$ of each other. This was consistent when considering that the difference between the total correction to the asymmetry calculated by regression with that calculated by dithering was $3.2 \pm 3.8$ ppb. Finally, the Møller detector asymmetry was found to be $-177.5 \pm 23.0 \mathrm{ppb}$ for Run I and $-141.9 \pm 21.9 \mathrm{ppb}$ for Run II.

\subsection{Corrections and Dilution Factors}

To obtain the parity violating physics asymmetry, the Møller detector measurements reported in the previous section had to be corrected for systematic asymmetries and dilution factors introduced by the beam, ep electrons, pions and other neutral backgrounds. The dilution factors come from the fact that the denominator in Eq. 6.1 also includes all the background contributions to the measured asymmetry. Finally, the Møller detector results had to be normalized by the beam polarization and detector linearity. If $A_{\text {meas }}$ is the Møller detector asymmetry, the parity-violating asymmetry $A_{P V}$ is given by

$$
A_{P V}=\frac{1}{P_{b} \epsilon} \frac{A_{\text {meas }}-\sum_{i} \Delta A_{i}}{1-\sum_{i} f_{i}},
$$

where $\Delta A_{i}$ is the asymmetry correction and $f_{i}$ is the dilution factor for a given background $i . \quad P_{b}$ is the beam polarization and $\epsilon$ is the linearity of the detector response.

Another important task was to estimate the statistical and systematic uncertainty on the parity-violating asymmetry. The statistical uncertainty to $A_{P V}$ was obtained from evaluating the uncertainty on the Møller detector measurement. The systematic uncertainty, on the other hand, came from a combination of the uncertainties in estimating the various backgrounds. Section 6.3 is devoted to identifying and esti- 
mating the background corrections and dilution factors, and their contribution to the systematic uncertainty.

\subsubsection{Beam Systematic Uncertainties}

Beam systematic uncertainties were divided into first-order and higher-order contributions. Before explaining how they were estimated, it is necessary to introduce the concept of dipoles. The Møller detector rings exhibited a sinusoidal azimuthal variation in the left-right asymmetry (Figure 6.4). This effect can be studied by calculating the dipole asymmetry for a ring given by

$$
A_{X \text { dipole }}=\frac{2}{N_{\text {ring }}} \sum_{i} A_{i} \sin \left(\frac{2 \pi\left(i-\phi_{\text {ring }}\right)}{N_{\text {ring }}}\right) \text {, }
$$

where $A_{i}$ is the channel $i$ left-right asymmetry, $N_{\text {ring }}$ is the number of channels in that ring, $\phi_{\text {ring }}$ is the phase of the top channel of that ring, such that if the top channel is perfectly aligned with the y-direction $\phi_{\text {ring }}=0 . A_{X \text { dipole }}$ is the dipole asymmetry in the x-direction. Similarly, the dipole in the y-direction $A_{Y \text { dipole }}$ is given by

$$
A_{Y \text { dipole }}=\frac{2}{N_{\text {ring }}} \sum_{i} A_{i} \cos \left(\frac{2 \pi\left(i-\phi_{\text {ring }}\right)}{N_{\text {ring }}}\right) .
$$

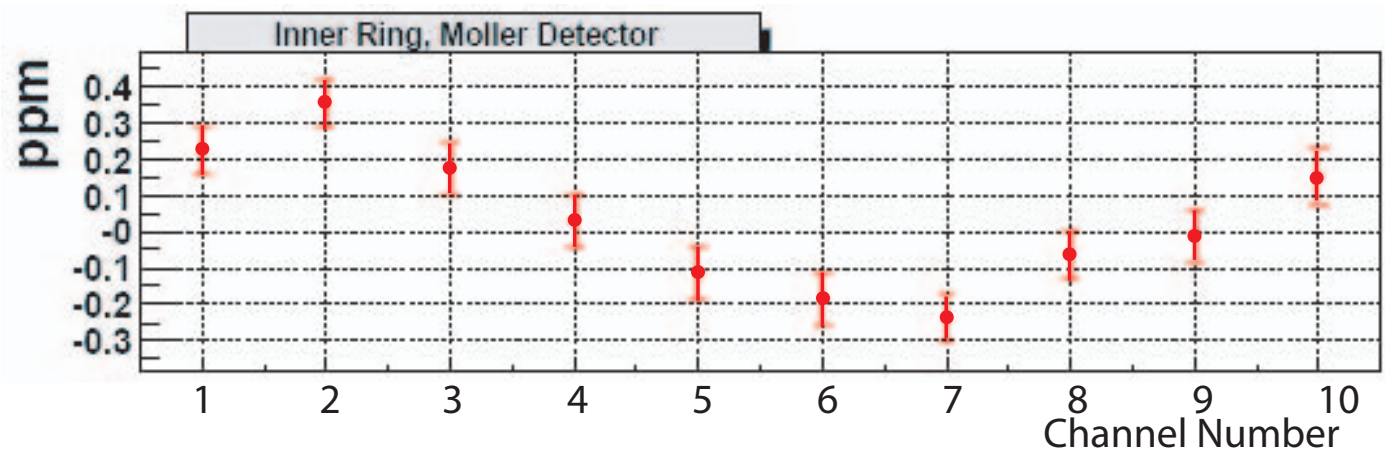

Figure 6.4: The Møller asymmetry per channel versus channel (azimuth) for the inner ring.

The dipoles were induced by mainly two effects. First, if the beam energy was not set precisely to 45.0 or $48.3 \mathrm{GeV}$, there would be a small amount of horizontal 
transverse polarization in the beam. This could produce two-photon exchange processes which would induce an azimuthal asymmetry in the Møller detector, although the average asymmetry around the ring would be zero. This phenomenon and its contribution to the physics asymmetry were studied by running the beam at 46.6 and $42 \mathrm{GeV}$, where the beam polarization was fully transverse. From these studies it was estimated that the contribution due to transverse beam polarization was $-8 \pm 3 \mathrm{ppb}$ for Run I and $-5 \pm 3 \mathrm{ppb}$ for Run II, a small correction.

The second and dominant effect that gave rise to dipoles was due to imperfections in the beam corrections. For example, the channels that were closer to the horizontal plane tended to be more sensitive to the asymmetries in the $\mathrm{x}$ position and $\mathrm{x}$ angle. If the $\mathrm{x}$ position and $\mathrm{x}$ angle corrections to the asymmetry were done systematically incorrectly, then the horizontal channels would exhibit larger asymmetries compared to the vertical channels. Furthermore, the asymmetries for the horizontal channels at opposite sides would have opposite signs. In order to study systematic uncertainty contributions for each beam parameter, one could select the dipole out of the three detector rings that was the most sensitive to that beam parameter. The ring combinations used to study first-order beam systematic uncertainties are given in Table 6.2. Note that for the energy correction the overall Møller detector asymmetry was used.

\begin{tabular}{|c|l|}
\hline Beam Parameter & Asymmetry \\
\hline $\mathrm{E}$ & Møller detector asymmetry \\
\hline $\mathrm{x}$ & Xdipole of middle ring \\
\hline $\mathrm{y}$ & Ydipole of middle ring \\
\hline $\mathrm{x}$ angle & Xdipole of outer ring \\
\hline $\mathrm{y}$ angle & Ydipole of outer ring \\
\hline
\end{tabular}

Table 6.2: Various ring combinations used to study first-order beam systematic uncertainties for each beam parameter.

The first-order beam systematic uncertainties were calculated as follows: First, the overall correction to the Møller detector asymmetry for each beam parameter was computed by using the regression slopes and the asymmetry of the beam parameter (see Eq. 6.2). Second, the relative error of that correction was estimated by taking the ratio of the timeslot difference of the dipole asymmetry sensitive to that parameter 
to the timeslot difference of that parameter's correction:

$$
\text { relative error }=\frac{\partial A}{\partial \text { correction }}=\frac{A_{\text {timeslot } 0}-A_{\text {timeslot } 1}}{\text { correction }_{\text {timeslot } 0}-\text { correction }_{\text {timeslot } 1}} .
$$

For example, the overall correction to the Møller detector asymmetry during Run II due to x position was $4.5 \mathrm{ppb}$. The relative error, calculated using the Xdipole middle ring asymmetries, was $3.8 \%$. Multiplying the two, the $\mathrm{x}$ position contribution to firstorder beam systematic uncertainties was found to be $0.17 \mathrm{ppb}$. The total contribution from all beam parameters was obtained by adding each parameter contribution in quadrature. This method assumed that systematic uncertainty contributions from different beam parameters were uncorrelated. The reason it was used is because it produced the most conservative estimate. Using this procedure it was found that first-order beam systematic uncertainties were $\sim 3 \mathrm{ppb}$ for Run I and $\sim 2 \mathrm{ppb}$ for Run II.

The higher-order beam systematic uncertainties were obtained from an analysis of the outer ring of the Møller detector [36]. It turned out that in addition to the six beam parameters (charge, energy, $\mathrm{x}$ and $\mathrm{y}$ position and angle), this ring was particularly sensitive to other, unmeasured beam parameters, which were collectively called "higher-order beam effects". In particular, the outer Møller detector channels were sensitive to changes in beam parameters within the duration of one beam pulse. These changes were undetected by the BPMs and toroids, which integrated over the entire length of the pulse. During Run III the BPMs signals were "sliced" in order to measure the beam asymmetry as a function of time within one pulse. Regressing against the sliced signals proved to be an adequate method for estimating and removing higher-order beam effects. Combining what was learned from the "slice" regression during Run III with the outer ring analysis for Run I and Run II, the uncertainties due to higher-order beam effects were estimated to be $\sim 10$ ppb and $\sim 15$ ppb for Run I and Run II, respectively. 


\subsubsection{Systematic Uncertainties from Beam Spot Size Asymmetries}

The beam spot size was defined as $S=\pi \sigma_{x} \sigma_{y}$ where $\sigma_{x}$ and $\sigma_{x}$ were obtained from a Gaussian fit to the wire array $\mathrm{x}$ and $\mathrm{y}$ beam profiles, respectively. Asymmetries and fluctuations in the beam spot size caused asymmetries and fluctuations in the liquid hydrogen target density, which in turn led to systematic uncertainties in the measured Møller detector asymmetry. These contributions were small given that spot size asymmetries were found to be consistent with zero. In order to estimate them, however, a correlation was found for every run by correlating the Møller detector asymmetry to the beam spot size. Then, the spot size contribution to the asymmetry for a run is given by [69]

$$
\Delta A_{\text {spotsize }}=\alpha\left[\pi \sigma_{x}\left\langle D_{y}\right\rangle+\pi \sigma_{y}\left\langle D_{x}\right\rangle\right]
$$

where $\alpha$ is the correlation for that run and $\left\langle D_{y}\right\rangle$ and $\left\langle D_{y}\right\rangle$ are the left-right spot size differences in $\mathrm{x}$ and $\mathrm{y}$, respectively. The overall contribution was calculated by averaging $\Delta A_{\text {spotsize }}$ over all runs and resulted in $0.06 \pm 0.5 \mathrm{ppb}$, which was consistent with zero. Conservatively, a 1 ppb systematic contribution was assigned to the spot size asymmetry.

\subsubsection{Electron-Proton Background}

In addition to Møller electrons, the Møller detector was hit by electrons originating from electron-proton interactions (ep electrons), which also exhibited a helicitycorrelated left-right asymmetry. The ep electrons not only shifted the overall value of the measured asymmetry, but also diluted its value by contributing to the signal recorded by the Møller channels. To estimate the ep asymmetry and dilution factors, the results from the ep detector were used in conjunction with profile detector scans taken at various settings of collimators in and out of the beam, and quadrupoles on and off. The scan results were compared to a Monte Carlo simulation, designed specifically for the experiment's conditions [8]. 


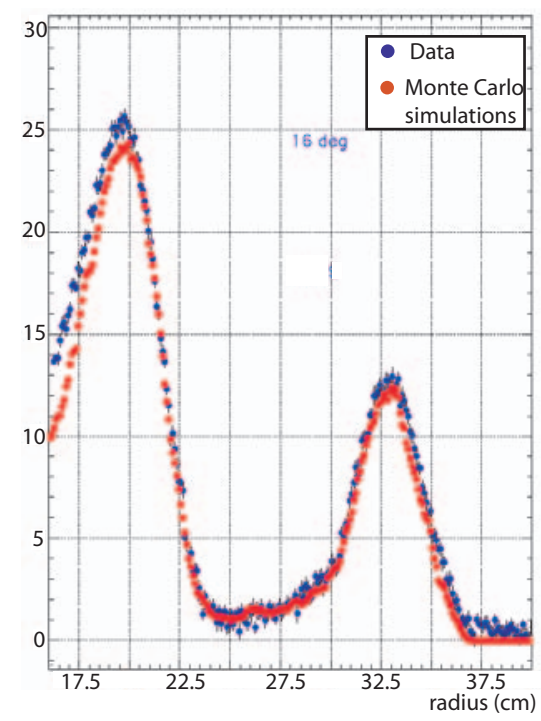

Figure 6.5: A profile detector scan taken with the "holey" collimator inserted. Also shown is the Monte Carlo simulation, normalized to the data sample.

An example of a profile detector scan and the Monte Carlo simulation is given in Figure 6.5. For this particular scan the "holey" collimator, described in Section 4.2.4 was inserted, which created a very clean separation of the Møller and ep flux at the face of the detector. The Monte Carlo simulation was normalized to the data sample, and its results were used to determine the ep flux in the Møller detector. As a check, the data was also fit with a GEANT simulation [64], and both simulations agreed well. A detailed description of the procedure for estimating both the elastic and inelastic ep backgrounds is given in Reference [5]. The results are tabulated in Table 6.3

\begin{tabular}{|c|c|c||c|c|}
\hline \multirow{2}{*}{} & \multicolumn{2}{|c||}{ 45 GeV } & \multicolumn{2}{c|}{ 48 GeV } \\
\cline { 2 - 5 } & correction (ppb) & dilution & correction (ppb) & dilution \\
\hline Run I & $-32.6 \pm 5.0$ & $0.781 \pm 0.0086$ & $-34.4 \pm 5.4$ & $0.0700 \pm 0.0077$ \\
\hline Run II & $-27.5 \pm 4.1$ & $0.0637 \pm 0.0077$ & $-29.5 \pm 4.1$ & $0.0614 \pm 0.0062$ \\
\hline
\end{tabular}

Table 6.3: Total (elastic+inelastic) ep asymmetry corrections and dilution factors. 


\subsubsection{Pion Corrections}

The effect of the pion background to the parity-violating physics asymmetry is given by [39]

$$
A_{\text {meas }}=A_{P V} \times\left(1-\frac{\epsilon N_{\pi}}{N_{e}}\right)+\frac{\epsilon N_{\pi}}{N_{e}} A_{\pi}
$$

where $A_{\text {meas }}$ and $A_{\pi}$ are the asymmetries measured by the Møller and the pion detector, respectively. $N_{\pi}$ and $N_{e}$ are the rates of pions and electrons in the Møller detector, and $\epsilon$ is the ratio of the energy deposited by the pions to that deposited by the electrons in the Møller detector. From Eq. 6.18 the asymmetry correction due to pions is

$$
\Delta A_{\pi}=\frac{\epsilon N_{\pi}}{N_{e}} \times A_{\pi}
$$

The pion detector recorded an asymmetry of $A_{\pi}=-0.36 \pm 0.48$ (stat) ppm. The

flux ratio $\frac{N_{\pi}}{N_{e}}$ and the energy ratio $\epsilon$ were estimated via a GEANT simulation to be $0.0063 \pm 0.0021$ and $0.22 \pm 0.15$, respectively. From Eq. 6.19 one obtains that $\Delta A_{\pi}=1.0 \pm 1.0 \mathrm{ppb}$ and the dilution factor, which is just $\epsilon \frac{N_{\pi}}{N_{e}}$, is $0.001 \pm 0.001$ [39]

\subsubsection{Corrections due to Neutral Backgrounds}

The neutral backgrounds in the Møller detector include high-energy photons (mostly multi-bounce photons from scattering off collimators), residual synchrotron photons, neutral hadrons generated in the detector, spectrometer beampipe splash (photons generated by high-energy electrons hitting the spectrometer beampipe), and leakage from the ep detector. The ep detector leakage was due to ep electron showers spreading from the ep detector into the Møller detector. The collimator masks in the drift pipe region (see Section 4.2.6), which were added after Run I, prevented many of the backgrounds from hitting the detector. As a result, during Run II, backgrounds were reduced by a factor of four.

To estimate the neutral backgrounds, data was taken in five different configurations, each of which blocked or enhanced a combination of the neutral backgrounds [35]. The combinations and their background sensitivities are listed below: 
1. Quadrupoles off, all collimator masks removed. In this configuration all neutral backgrounds contributed.

2. Quadrupoles off, all collimator masks inserted, except for the mask furthest downstream. The upstream collimator masks were only able to block the backgrounds due to spectrometer beampipe splash. As a result, in this configuration all neutral backgrounds, except for the spectrometer beampipe splash, contributed.

3. Quadrupoles off, all collimator masks removed, "blinded" PMTs. Aluminum tape was inserted in front of the PMT lightguide, such that the signal recorded from the Møller detector was only due to neutral hadrons that penetrated to the cathodes.

4. Removing the liquid hydrogen target. In this configuration the Møller signal was dominated by synchrotron radiation.

5. Quadrupoles off, all collimator masks inserted. The only contributions in this case were from high energy photons and synchrotron radiation.

Table 6.4 gives the asymmetry corrections and dilution factors due to neutral backgrounds [38]. Some of the above-mentioned backgrounds were found to have insignificant contributions, and are not included in the table.

\begin{tabular}{|c|c|c||c|c|}
\hline \multirow{2}{*}{ Source } & \multicolumn{2}{|c||}{ Run I } & \multicolumn{2}{c|}{ Run II } \\
\cline { 2 - 5 } & $\Delta \mathbf{A} \mathbf{~ ( p p b )}$ & $\mathbf{f}$ & $\Delta \mathbf{A} \mathbf{~ ( p p b )}$ & $\mathbf{f}$ \\
\hline High energy photons & $3 \pm 3$ & $0.004 \pm 0.002$ & $3 \pm 3$ & $0.004 \pm 0.002$ \\
\hline Synchrotron photons & $0 \pm 5$ & $0.002 \pm 0.001$ & $0 \pm 2$ & $0.002 \pm 0.001$ \\
\hline Neutrons & $-5 \pm 3$ & $0.003 \pm 0.001$ & $0 \pm 0$ & $0.000 \pm 0.000$ \\
\hline
\end{tabular}

Table 6.4: Corrections and dilution factors due to neutral backgrounds.

\subsubsection{Linearity of the Møller Detector Response}

One way of estimating the Møller detector linearity $\epsilon$ was to compare detector asymmetries obtained under different flux conditions. If $F$ is the Møller flux, the measured 
asymmetry is related to the parity violating asymmetry by [13]

$$
A_{\text {meas }}=A_{P V}\left(1-\beta F+\mathcal{O}(\beta F)^{2}\right),
$$

where $\beta$ is a constant and $\epsilon=1-\beta F$ is the detector linearity. In order to create a higher $A_{\text {meas }}$ than $A_{P V}$, the polarized foil target was inserted. The Møller flux was varied by running in three different states: PMTs uncovered, PMTs covered with aluminum foil with a one-inch hole punch through it, and PMTs covered with aluminum with a half-inch hole punched though. The linearity was obtained by taking the asymmetry ratio between high-flux and low-flux states:

$$
\frac{A_{\text {meas }}^{\text {high }}}{A_{\text {meas }}^{\text {low }}}=\frac{1-\beta F^{\text {high }}}{1-\beta F^{\text {low }}} \approx 1-\beta F^{\text {high }}
$$

where the assumption has been made that at low flux level the detector response was very linear and $\beta F^{\text {low }} \ll 1$. The assumption was based on bench measurements of the linearity of the PMT response. Using this procedure, it was determined that Møller detector linearity was $0.99 \pm 0.01$ for both Run I and Run II data.

\subsubsection{Beam Polarization}

As described in Section 5.4 the beam polarization was obtained from measuring the asymmetry produced when the beam hit the polarized foil target. However, the asymmetry that the polarimeter detector measured, $A_{\text {polarization }}^{\text {meas }}$ had to be corrected for backgrounds in order to extract the physics polarization asymmetry $A_{\text {polarization. }}$ The backgrounds consisted primarily of ep electrons and low-energy photons. Background contributions were estimated by fitting a GEANT Monte Carlo simulation to radial scans of the polarimeter detector, and comparing the data taken with the polarized foil target in and out of the beam [12]. The backgrounds were found to be $8 \% \pm 3 \%$ of the asymmetry measured by the polarimeter detector.

After correcting for backgrounds, the beam polarization was determined for each polarimetry measurement, which took place on alternate days. The polarization 
values from each measurement were extremely close to each other, signifying a stable beam polarization during the experiment. Averaging over all measurements, the beam polarization was found to be $85 \% \pm 5 \%$ for Run I and $84 \% \pm 5 \%$ for Run II. The $5 \%$ uncertainty in the polarization measurement was obtained by adding in quadrature all systematic contributions to the polarization measurement. The biggest contributions were due to background subtraction $(3 \%)$ and the measurement of the foil target magnetization $(3 \%)$

\subsubsection{Luminosity Monitor Results}

The luminosity monitor measured the very forward angle Møller and ep electron asymmetry. According to a Monte Carlo simulation, the luminosity monitor was expected to measure an asymmetry of $-15 \pm 5 \mathrm{ppb}$ (Figure 6.6). The simulation

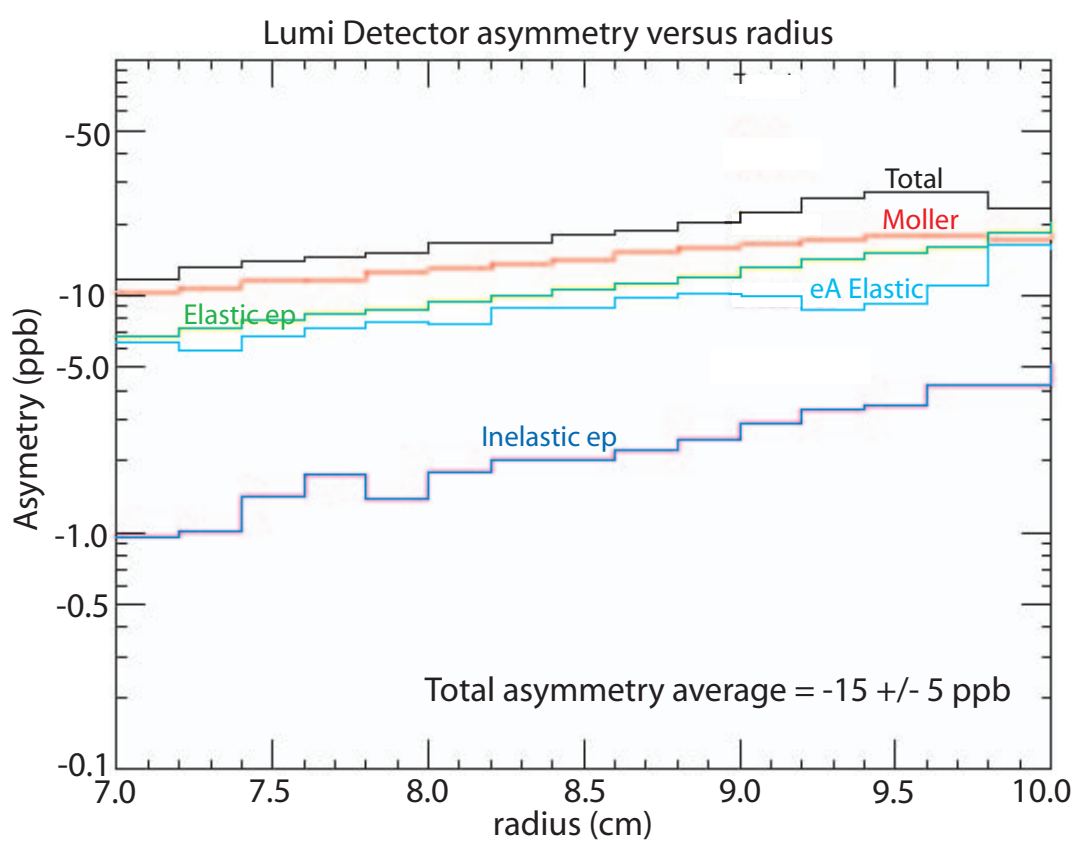

Figure 6.6: A Monte Carlo simulation of the expected asymmetry at the luminosity monitor. Contributions from the Møller and ep electrons, and from electrons scattering off the target aluminum windows (eA Elastic) are shown together with their total sum.

took into account contributions to the asymmetry from the Møller electrons, elastic and inelastic ep electrons, and electrons scattered off the target aluminum windows. 
The luminosity monitor analysis was very similar to that of the Møller detector. The data was regressed to correct for helicity-correlated beam asymmetries and the same cuts as for the Møller detector data were applied. The luminosity monitor measured an asymmetry of $-16 \pm 15 \mathrm{ppb}$ for Run I and $-14 \pm 12$ for Run II [34], as shown in Figure 6.7. The results are consistent with the prediction from the Monte Carlo simulation, another indication that there were no significant higher-order systematic uncertainties.
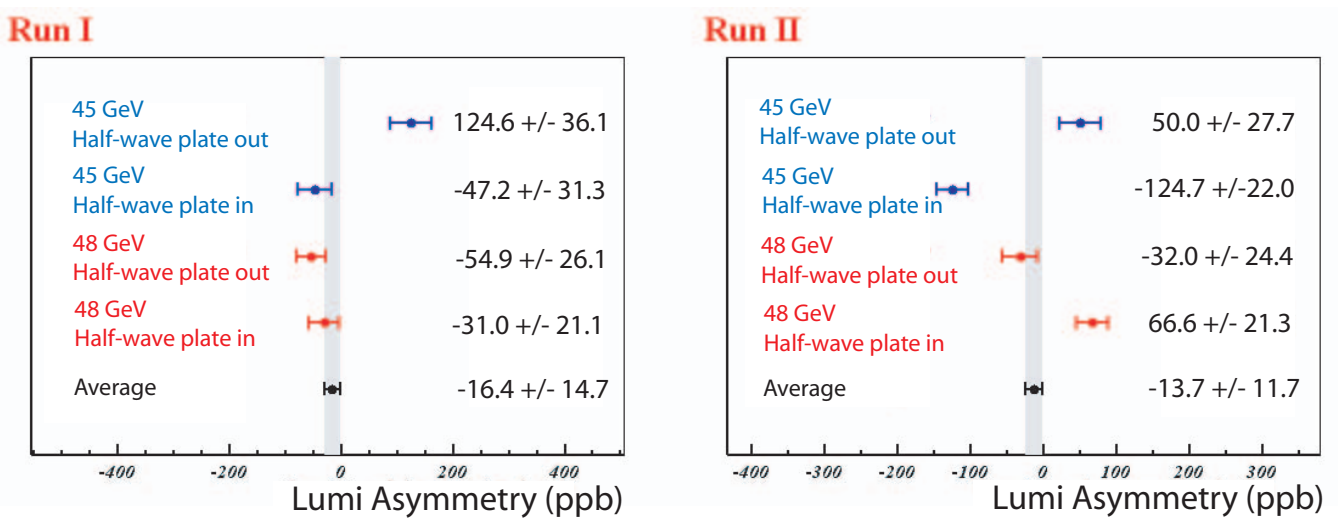

Figure 6.7: Luminosity monitor asymmetry for the four different spin-flip combinations and the overall average for Run I and Run II. The plots demonstrate how averaging over asymmetry reversals suppressed systematic effects.

\subsection{The Parity-Violating Asymmetry}

A summary of all the asymmetry corrections and the dilution factors for both Run I and Run II is given in Table $6.5^{2}$. The parity-violating asymmetry is calculated separately for each energy using Eq. 6.13 and the average Møller detector asymmetries for 45 and $48 \mathrm{GeV}$. The overall asymmetry is given by the weighted sum of the 45 and $48 \mathrm{GeV}$ data. The weight is obtained from the statistical uncertainty at each energy,

\footnotetext{
${ }^{2}$ In this Table the $1^{\text {st }}$ order beam contribution, $\Delta \mathrm{A}$, is zero because, due to regression, it is already taken into account in the Møller detector asymmetry. The $1^{\text {st }}$ order beam contribution removed by regression was $-41 \mathrm{ppb}$ for Run I and $-19 \mathrm{ppb}$ for Run II.
} 


\begin{tabular}{|c|c|c|c|c|}
\hline \multirow[b]{2}{*}{ Source } & \multicolumn{2}{|c|}{$\begin{array}{c}\text { Run I } \\
\mathrm{A}_{\text {meas }}=-177.5 \pm 23.0 \mathrm{ppb}\end{array}$} & \multicolumn{2}{|c|}{$\begin{array}{c}\text { Run II } \\
\mathrm{A}_{\text {meas }}=-141.9 \pm 21.9 \mathrm{ppb}\end{array}$} \\
\hline & $\Delta \mathrm{A}(\mathrm{ppb})$ & f & $\Delta \mathrm{A}(\mathrm{ppb})$ & $\mathbf{f}$ \\
\hline Beam $1^{\text {st }}$ order & $0 \pm 3$ & & $0 \pm 2$ & \\
\hline Beam $2^{\text {nd }}$ order & $0 \pm 10$ & & $0 \pm 15$ & \\
\hline Beam spot size & $0 \pm 1$ & & $0 \pm 1$ & \\
\hline Transverse polarization & $-8 \pm 3$ & & $-5 \pm 3$ & \\
\hline ep total & $-33 \pm 5$ & $0.075 \pm 0.008$ & $-29 \pm 4$ & $0.062 \pm 0.007$ \\
\hline High energy photons & $3 \pm 3$ & $0.004 \pm 0.002$ & $3 \pm 3$ & $0.004 \pm 0.002$ \\
\hline Synchrotron photons & $0 \pm 5$ & $0.002 \pm 0.001$ & $0 \pm 2$ & $0.002 \pm 0.001$ \\
\hline Neutrons & $-5 \pm 3$ & $0.003 \pm 0.001$ & $0 \pm 0$ & $0.000 \pm 0.000$ \\
\hline Pions & $1 \pm 1$ & $0.001 \pm 0.001$ & $1 \pm 1$ & $0.001 \pm 0.001$ \\
\hline
\end{tabular}

Table 6.5: Corrections and dilution factors for Run I and Run II.

which is just the uncertainty of the Møller detector asymmetry at that energy:

$$
\begin{gathered}
\text { weight }_{45 \mathrm{GeV}}=\frac{\sigma_{45 \mathrm{GeV}}^{-2}}{\sigma_{45 \mathrm{GeV}}^{-2}+\sigma_{48 \mathrm{GeV}}^{-2}} \\
A_{P V}=\frac{A_{45 \mathrm{GeV}}^{P V} \times \text { weight }_{45 \mathrm{GeV}}+A_{48 \mathrm{GeV}}^{P} \times \text { weight }_{48 \mathrm{GeV}}}{\text { weight }_{45 \mathrm{GeV}}+\text { weight }_{48 \mathrm{GeV}}},
\end{gathered}
$$

where weight ${ }_{45 \mathrm{GeV}}$ and $\sigma_{45 \mathrm{GeV}}$ are the weight and the statistical uncertainty at 45 GeV. Similarly, weight $48 \mathrm{GeV}$ and $\sigma_{48 \mathrm{GeV}}$ are the weight and the statistical uncertainty at $48 \mathrm{GeV}$.

The overall statistical uncertainty is given by

$$
\sigma_{\text {stat }}=\left(\frac{1}{\sigma_{45}^{2}}+\frac{1}{\sigma_{48}^{2}}\right)^{-1 / 2}
$$

The overall systematic uncertainty is calculated from the uncertainty in corrections, dilutions and normalization, where the normalization is just polarization $\times$ linearity $=P_{b} \epsilon$. The uncertainty in the corrections is

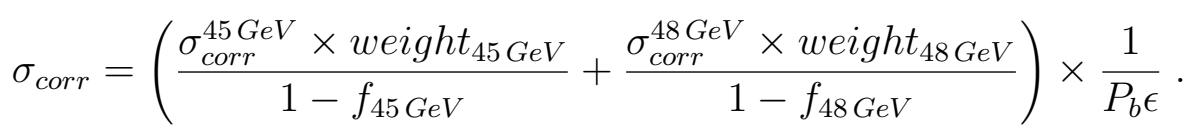


The uncertainty in the dilutions is

$$
\sigma_{f}=\left(\text { weight }_{45 \mathrm{GeV}} \times \frac{\sigma_{f}^{45 \mathrm{GeV}} \times A_{45 \mathrm{GeV}}^{P V}}{1-f_{45 \mathrm{GeV}}}\right)+\left(\text { weight }_{48 \mathrm{GeV}} \times \frac{\sigma_{f}^{48 \mathrm{GeV}} \times A_{48 \mathrm{GeV}}^{P V}}{1-f_{48 \mathrm{GeV}}}\right) .
$$

Since the linearity and polarization were the same for both energies, the normalization uncertainty is calculated as

$$
\sigma_{\text {normalization }}=\frac{\sqrt{\left(\sigma_{\epsilon} P_{b}\right)^{2}+\left(\sigma_{P_{b}} \epsilon\right)^{2}}}{P_{b} \epsilon} \times A_{P V}
$$

The systematic uncertainty is then

$$
\sigma_{\text {syst }}=\sqrt{\sigma_{\text {corr }}^{2}+\sigma_{f}^{2}+\sigma_{\text {normalization }}^{2}} .
$$

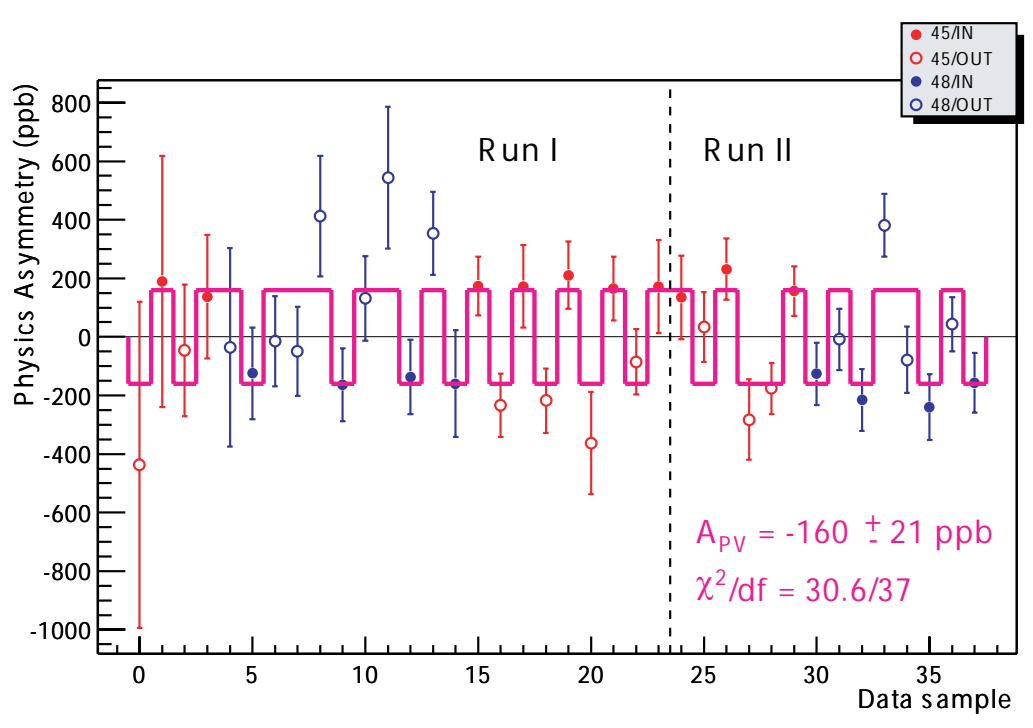

Figure 6.8: The parity violating asymmetry for each slug for both Run I and Run II. The data has not been corrected for asymmetry sign flips and the pink solid line represents the grand average with the expected asymmetry sign for each sign flip configuration. The uncertainty shown is only the statistical uncertainty.

A plot of the parity-violating asymmetry for each slug for both Run I and Run II is given in Figure 6.8. Using the above equations, the parity-violating physics asymmetry observed during experiment E158 was found to be 
Run I:

$-175 \pm 30$ (stat.) \pm 20 (syst.) $\mathrm{ppb}$

Run II:

$$
-144 \pm 28 \text { (stat.) } \pm 23 \text { (syst.) ppb }
$$

Run I+II combined: $-160 \pm 21$ (stat.) \pm 17 (syst.) ppb

The combined result for Run I and Run II establishes parity-violation in Møller scattering at the $6 \sigma$ level and is the most precise measurement to date of any asymmetry in electron scattering. 


\section{Chapter 7}

\section{The Weak Mixing Angle and Final Conclusions}

\subsection{Calculating the Weak Mixing Angle}

As shown in Chapter 1, the weak mixing angle is correlated to the parity-violating asymmetry in Møller scattering. The correlation is given by Eq. 1.3, which can be rewritten as [14]

$$
A_{P V}=\frac{G_{F} Q^{2}}{\sqrt{2} \pi \alpha} \frac{1-y}{1+y^{4}+(1-y)^{4}} \mathcal{F}_{b} Q_{w}^{e} \equiv \mathcal{A}\left(Q^{2}, y\right) Q_{w}^{e}
$$

where $y \equiv \frac{Q^{2}}{s}$, and $\sqrt{s}=\left[(k+p)^{2}\right]^{1 / 2}$ with $k$ and $p$ denoting the four-momenta of the incoming electrons in the $e^{-} e^{-} \rightarrow e^{-} e^{-}$scattering. The $\mathcal{F}_{b}$ factor accounts for radiative corrections to the parity-violating asymmetry due to bremsstrahlung contribution coming from inelastic processes that result in a real photon emission $e^{-} e^{-} \rightarrow e^{-} e^{-} \gamma$ (Figure 7.1). The quantity $Q_{w}^{e}$ is the weak charge, defined as $Q_{w}^{e} \equiv$ $1-4 \sin ^{2} \theta_{w}$. The quantity $\mathcal{A}\left(Q^{2}, y\right)$ is known as the analyzing power and depends on the specific experimental environment, such as beam parameters, target thickness, spectrometer acceptance, and detector response.

The analyzing power of the E158 experiment, $\mathcal{A}\left(Q^{2}, y\right)$, was calculated with the help of the same GEANT simulation [64], which was used to fit the profile detector scans and determine the ep background (see Section 6.3.2). However, the simulation did not incorporate the bremsstrahlung effects, which were calculated for the partic- 

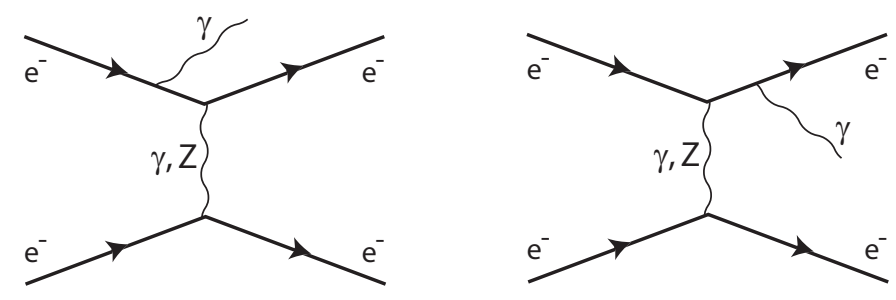

+ crossed diagrams
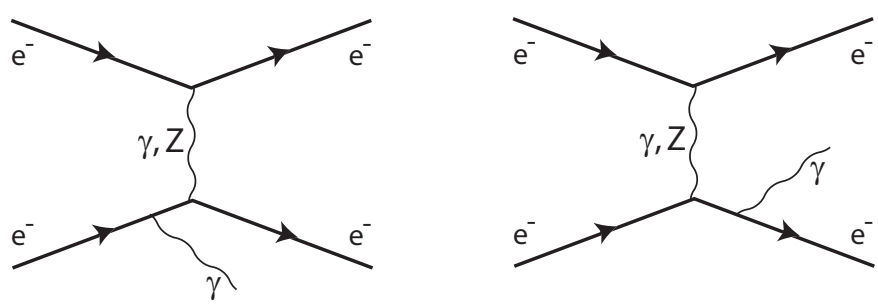

Figure 7.1: Bremsstrahlung contributions to the analyzing power.

ular kinematics of the E158 experiment by Zykunov [71] to be $\mathcal{F}_{b}=1.01 \pm 0.01$. The bremsstrahlung-corrected analyzing powers are given in Table 7.1 for Run I and II, as well for both Runs combined. The average values of the kinematic variables for the E158 experiment were found to be $Q^{2}=0.026(\mathrm{GeV} / \mathrm{c})^{2}$ and $y \simeq 0.6[2]$.

\begin{tabular}{|c|c|c|c|}
\hline & $45 \mathrm{GeV}$ & $48 \mathrm{GeV}$ & overall \\
\hline Run I (ppm) & $3.19 \pm 0.06$ & $3.41 \pm 0.05$ & $3.28 \pm 0.06$ \\
\hline Run II (ppm) & $3.25 \pm 0.05$ & $3.41 \pm 0.05$ & $3.34 \pm 0.05$ \\
\hline Run I+II combined (ppm) & $3.22 \pm 0.05$ & $3.40 \pm 0.05$ & $3.31 \pm 0.05$ \\
\hline
\end{tabular}

Table 7.1: Analyzing powers for Run I and Run II given for each energy, and averaged over an entire Run.

The theoretical predictions of the weak charge and weak mixing angle at $Q^{2}=$ $0.026(\mathrm{GeV} / \mathrm{c})^{2}$ are [14] [27]

$$
\begin{aligned}
Q_{w}^{e} & =-0.046 \pm 0.002 \\
\sin ^{2} \theta_{w \overline{M S}} & =0.2385 \pm 0.0006
\end{aligned}
$$

The prediction for the weak mixing angle is given in the modified minimal subtraction $(\overline{M S})$ scheme at the scale $\mu=m_{Z}$, where $\mu$ is the parametrization scale and $m_{Z}$ is the mass of the $Z$ boson. In this scheme the weak mixing angle is defined as the ratio 
of the $\overline{M S}$ electromagnetic and weak couplings: $\sin ^{2} \theta_{w}\left(m_{Z}\right) \overline{M S} \equiv \frac{g_{e}^{2}\left(m_{Z}\right) \overline{M S}}{g_{w}^{2}\left(m_{Z}\right) \overline{M S}}[41]$. Another way of expressing the weak mixing angle is known as the effective weak mixing angle $\sin ^{2} \theta_{w}^{e f f}$, defined by the ratio of vector and axial vector components for the on-mass-shell $Z \mu \mu^{-}$vertex: $1-4 \sin ^{2} \theta_{w}^{\text {eff }} \equiv \frac{g_{V}}{g_{A}}[21]$. The $\overline{M S}$ and effective schemes differ by finite $\mathcal{O}(\alpha)$ loop corrections, numerically given by [27]

$$
\sin ^{2} \theta_{w}^{e f f}=\sin ^{2} \theta_{w} \overline{M S}+0.00029
$$

The analyzing powers for the E158 experiment were calculated in the $\overline{M S}$ scheme. All results in this document are also presented in the $\overline{M S}$ scheme. The weak charge and weak mixing angle were obtained from Eq. 7.1, using the analyzing powers given in Table 7.1, and the parity-violating asymmetries calculated in Section 6.4. The values for $\sin ^{2} \theta_{w}$ were calculated separately for each energy, with the overall average given by a weighted sum of the 45 and $48 \mathrm{GeV}$ data, similar to the calculation of the overall $A_{P V}$ (see Eq. 6.22 and 6.23). The results for Run I and Run II are listed in Table 7.2. Combining both Runs, the weak charge and weak mixing angle at $Q^{2}=0.026(\mathrm{GeV} / \mathrm{c})^{2}$ were found to be

$$
\begin{aligned}
Q_{w}^{e} & =-0.048 \pm 0.006 \text { (stat.) } \pm 0.005 \text { (syst.) } \\
\sin ^{2} \theta_{w \overline{M S}} & =0.2379 \pm 0.0016 \text { (stat.) } \pm 0.0013 \text { (syst.) }
\end{aligned}
$$

in agreement with the Standard Model prediction given by Eq. 7.3. Using the result in Eq. 7.6 to extrapolate the weak mixing angle at the $Z^{0}$ pole [14] one finds

$$
\sin ^{2} \theta_{w}\left(m_{Z}\right)_{\overline{M S}}=0.2306 \pm 0.0016 \text { (stat.) } \pm 0.0013 \text { (syst.) }
$$

Comparisons between the E158 measurement of the weak mixing angle and measurements by other experiments are given in Figures 7.2 and 7.3. In Figure 7.2, the comparison is given in terms of the values of $\sin ^{2} \theta_{w}$ at the $Z^{0}$ pole. Figure 7.3 shows the experimental values and the theoretical prediction of the running of $\sin ^{2} \theta_{w}$ as a function of momentum transfer $Q$. (It is a repeat of Figure 1.1, with the addition of 
98

\begin{tabular}{|c|c|}
\hline & $Q_{w}^{e}$ \\
\hline Run I & $-0.053 \pm 0.009$ (stat.) \pm 0.006 (syst.) \\
\hline Run II & $-0.043 \pm 0.008$ (stat.) \pm 0.007 (syst.) \\
\hline \hline & $\sin ^{2} \theta_{w \overline{M S}}$ \\
\hline Run I & $0.2367 \pm 0.0023$ (stat.) \pm 0.0015 (syst.) \\
\hline Run II & $0.2392 \pm 0.0021$ (stat.) \pm 0.0017 (syst.) \\
\hline
\end{tabular}

Table 7.2: The weak charge and weak mixing angle measured at $Q^{2}=0.026(\mathrm{GeV} / \mathrm{c})^{2}$ during Run I and Run II. The results are -0.6 and +0.3 standard deviations away from the Standard Model prediction for Run I and Run II, respectively.

the E158 result.) As illustrated by Figures 7.2 and 7.3, the E158 result is consistent with theoretical predictions and with the results reported by the Particle Data Group (PDG2002) and the atomic parity violation experiment (Qw).

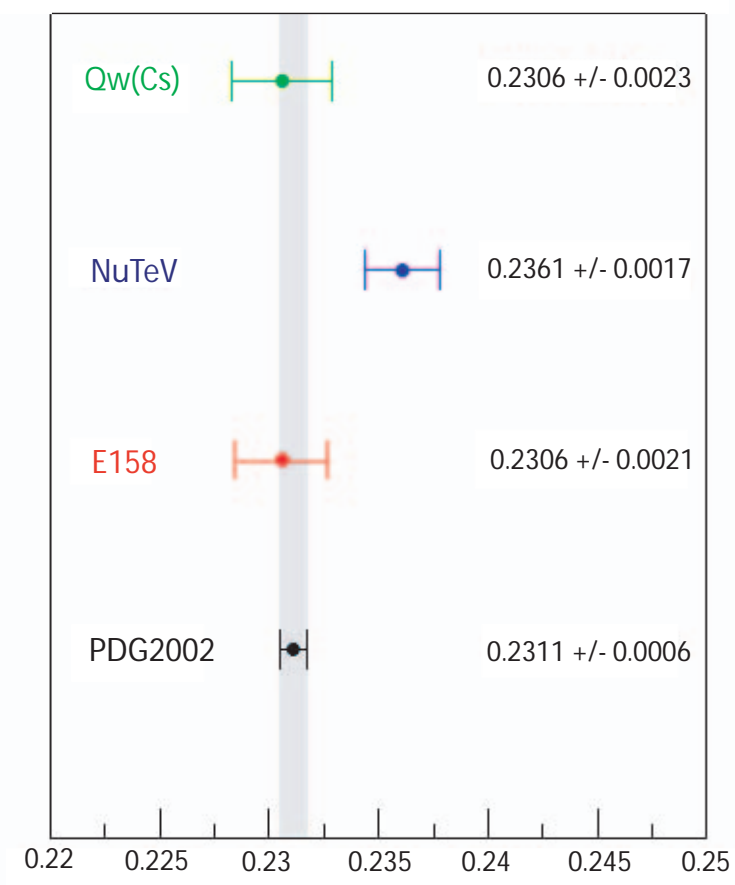

Figure 7.2: Measurements of the weak mixing angle at the $Z^{0}$ pole by the atomic parity violation experiment $(\mathrm{Qw}), \mathrm{NuTeV}$ experiment, E158 Experiment, and the LEP and SLD experiments (PDG2002). 


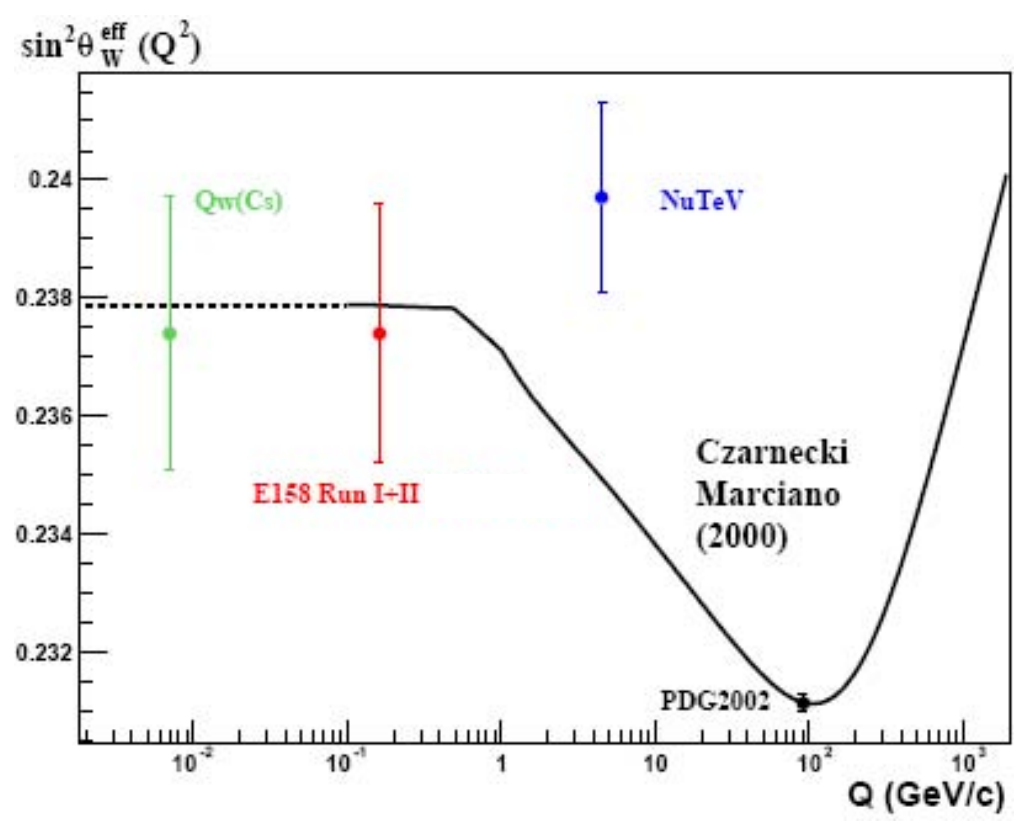

Figure 7.3: Measurements of $\sin ^{2} \theta_{w}$ as a function of momentum transfer $Q$. The solid line and the dotted line are the theoretical prediction at high $Q$ and low $Q$, respectively.

\subsubsection{New Physics Limits}

The E158 result for the weak mixing angle can be used to establish limits on certain classes of new physics, as described in Section 1.2.2. For example, the existence of additional neutral gauge bosons, known as $Z^{\prime}$, would affect the neutral current coupling, and consequently it would change the parity-violating asymmetry in Møller scattering. For an $\mathrm{SO}(10)$ model [14], $A_{P V}$ would increase by a factor of

$$
\frac{A_{P V}^{\text {meas }}}{A_{P V}^{S M}}=\frac{1-4 \sin ^{2} \theta_{w}^{\text {meas }}}{1-4 \sin ^{2} \theta_{w}^{S M}}=1+7 \frac{m_{Z}^{2}}{m_{Z^{\prime}}^{2}},
$$

where the superscripts meas and $S M$ stand for the measurement and the Standard Model prediction, respectively. The parameters $m_{Z}$ and $m_{Z^{\prime}}$ are the masses of the $Z$ and $Z^{\prime}$ bosons. Plugging into Eq. 7.8 the E158 measurement of $\sin ^{2} \theta_{w}$ (Eq. 7.6) and the Standard Model prediction (Eq. 7.3), a limit on the order of $\sim 1 \mathrm{TeV}$ can be set for the mass of $Z^{\prime}$.

Another limit can be placed on the compositeness scale for contact interaction 
among electrons, $\Lambda_{e e}$, since [10]

$$
\sin ^{2} \theta_{w}^{\text {meas }}-\sin ^{2} \theta_{w}^{S M}= \pm \frac{\pi}{G_{F} \sqrt{2}} \frac{\left(\eta_{R R}-\eta_{L L}\right)}{\Lambda_{e e}^{2}}
$$

where parameters $\eta_{R R}$ and $\eta_{L L}$ equal \pm 1 if there are parity-violating terms in the fourelectron contact interaction Lagrangian (see Section 1.2.2). At the 95\% confidence

level, the E158 result places a lower bound on $\Lambda_{e e}^{ \pm}$at $\sim 6$ and $\sim 7 \mathrm{TeV}$ for positive and negative deviations from the Standard Model, respectively. If a new four-electron contact interaction is mediated by a doubly charged Higgs boson $\Delta^{--}$, and if the scale of the interaction is much smaller than the mass of this boson $m_{\Delta}$, then [26] [55]

$$
\frac{g_{e e \Delta}^{2}}{m_{\Delta}^{2}} \approx \frac{4 \pi}{\Lambda_{e e}^{2}}
$$

where $g_{e e \Delta}$ is the $e e \Delta$ coupling. Using the upper limit for $\Lambda_{e e}$, one finds that $\frac{g_{e e \Delta}^{2}}{m_{\Delta}^{2}} \sim$ $0.3 \mathrm{TeV}^{-2}$, which is an order of magnitude improvement compared to the constraints coming from $(g-2)_{\mu}$ and muonium-antimuonium oscillation experiments [55].

The combined uncertainty of the E158 result for the weak mixing angle is $\delta\left(\sin ^{2} \theta_{w}\right)$ $=0.0020$. At this precision level, the limits on new physics set by the E158 experiment are competitive with limits set by the SLD and LEP collider experiments. With the addition of Run III data, the combined uncertainty for the entire 2002-2003 data collection period is expected to reduce to $\delta\left(\sin ^{2} \theta_{w}\right)=0.0015$, increasing the sensitivity to new physics.

\subsection{Future Experiments}

Measuring the running of the weak mixing angle is an important test of the electroweak theory. In order to confirm the Standard Model prediction for the correlation between $\sin ^{2} \theta_{w}$ and momentum transfer $Q$, more measurements at low $Q^{2}$ would be a valuable test of the electroweak theory and search for new interactions. Furthermore, since different experiments use different particles to measure $\sin ^{2} \theta_{w}$, their results are 
sensitive to a different set of radiative corrections and different new physics scenarios.

In the future, several experiments are planned for measuring $\sin ^{2} \theta_{w}$. The $Q_{w e a k}$ experiment (also known as JLab E-02-020) [3] is is scheduled to run in 2006 in Hall $\mathrm{C}$ at Jefferson Laboratory (JLab). The goal of the $Q_{\text {weak }}$ experiment is to measure the parity-violating asymmetry at forward angles using elastic electron-proton scattering at $Q^{2}=0.03(\mathrm{GeV} / \mathrm{c})^{2}$. From this asymmetry one can obtain the proton weak charge $Q_{w}^{p}$, which is related to the weak mixing angle through $Q_{w}^{p} \equiv 1-4 \sin ^{2} \theta_{w}$, similar to the definition of the electron weak charge $Q_{w}^{e}$ measured by the SLAC E158 experiment.

The parity-violating asymmetry $A_{P V}^{\text {Qweak }}$ is given by

$$
A_{P V}^{Q w e a k}=\frac{\sigma_{L}-\sigma_{R}}{\sigma_{L}+\sigma_{R}}
$$

where $\sigma_{L}$ and $\sigma_{R}$ are the scattering cross sections of left- and right-handed longitudinally polarized electrons, scattering off unpolarized protons. For forward angle scattering $A_{P V}^{Q w e a k}$ can be rewritten as

$$
A_{P V}^{Q w e a k} \cong\left[\frac{-G_{F}}{4 \pi \alpha \sqrt{2}}\right]\left[Q^{2} Q_{w}^{p}+Q^{4} B\left(Q^{2}\right)\right]
$$

where $Q$ and $Q_{w}^{p}$ denote the momentum transfer and the proton weak charge, respectively. The quantity $B\left(Q^{2}\right)$ is the leading term in the nucleon structure form factors contributing to $A_{P V}^{Q w e a k}$, and it is defined in terms of the electromagnetic and weak form factors of the proton and the neutron. The value of $B\left(Q^{2}\right)$ has been determined experimentally from previous forward angle parity-violating experiments at high $Q^{2}$, such as the HAPPEX experiment at JLab [28].

During the $Q_{w e a k}$ experiment, the parity-violating asymmetry will be measured by employing a $6 \mathrm{GeV}, 180 \mu \mathrm{A}$ continuous-mode electron beam with $80 \%$ polarization impinging upon a $35 \mathrm{~cm}$ long liquid hydrogen target. A toroidal magnetic field will focus the forward angle ep scatters onto a ring of eight rectangular quartz Cerenkov detectors. The Standard Model value for $A_{P V}^{Q w e a k}$ is expected to be $-280 \mathrm{ppb}$. The 
goal of the $Q_{\text {weak }}$ experiment is to measure this asymmetry to a precision of $\sim 4 \%$, which translates into a measurement of $\sin ^{2} \theta_{w}$ at $Q^{2}=0.03(\mathrm{GeV} / \mathrm{c})^{2}$ to a precision of $\sim 0.3 \%$.

If the $Q_{\text {weak }}$ experiment achieves the design precision, it will test new physics at scales up to $\sim 5 \mathrm{TeV}$. The $Q_{\text {weak }}$ results will be sensitive to new physics such as additional neutral gauge bosons, extensions to the Standard Model that include supersymmetry, and the existence of leptoquarks (bosons with nonzero baryon and lepton number). Finally, since the $Q_{\text {weak }}$ experiment will measure $\sin ^{2} \theta_{w}$ at approximately the same momentum transfer as the SLAC E158 experiment, the results from both experiments should complement each other and provide more clues regarding the nature of possible new physics phenomena.

Another experiment, known as the DIS-Parity experiment [1], has been proposed to run after the $12 \mathrm{GeV}$ energy upgrade at JLab. The experiment will perform a measurement of the parity-violating asymmetry of electron-deuteron deep inelastic scattering, similar to the SLAC E122 experiment [49], but with a high enough precision to compete with existing measurements of $\sin ^{2} \theta_{w}$. The electron-deuteron parity-violating asymmetry is given by

$$
A_{P V}^{D I S}=\frac{\sigma_{L}-\sigma_{R}}{\sigma_{L}-\sigma_{R}}=-\left(\frac{3 G_{F} Q^{2}}{2 \sqrt{2} \pi \alpha}\right) \frac{2 C_{1 u}-C_{1 d}\left[1+R_{s}(x)\right]+Y\left(2 C_{2 u}-C_{2 d}\right) R_{v}(x)}{5+R_{s}(x)}
$$

where $C_{1 u(d)}$ and $C_{1 u(d)}$ are the electron-quark electroweak couplings, approximately given by

$$
\begin{aligned}
C_{1 u} & =g_{A}^{e} g_{V}^{u}=-\frac{1}{2}+\frac{4}{3} \sin ^{2} \theta_{W} \\
C_{1 d} & =g_{A}^{e} g_{V}^{d}=\frac{1}{2}-\frac{2}{3} \sin ^{2} \theta_{W} \\
C_{2 u} & =g_{V}^{e} g_{A}^{u}=-\frac{1}{2}+2 \sin ^{2} \theta_{W} \\
C_{2 d} & =g_{V}^{e} g_{A}^{d}=\frac{1}{2}-2 \sin ^{2} \theta_{W} .
\end{aligned}
$$

Here $g_{V}$ and $g_{A}$ are the vector and axial couplings for electrons (e) and quarks $(\mathrm{u}, \mathrm{d})$. 
The quantity $Y$ in Eq. 7.13 is kinematics:

$$
Y=\frac{1-(1-y)^{2}}{1+(1-y)^{2}-y^{2} R /(1+R)}
$$

where $R \equiv \frac{\sigma_{L}}{\sigma_{R}}$ and $y$ is defined in Eq. 7.1. The ratios $R_{s}$ and $R_{v}$ are obtained from the quark distribution functions:

$$
R_{s}(x)=\frac{s(x)+\bar{s}(x)}{u(x)+\bar{u}(x)+d(x)+\bar{d}(x)} \text { and } \frac{u_{V}(x)+d_{V}(x)}{u(x)+\bar{u}(x)+d(x)+\bar{d}(x)},
$$

where $u_{V}(x)$ and $d_{V}(x)$ are the valence quark distributions, $s(x)$ and $\bar{s}(x)$ are the sea quark distributions, and $u(x)=u_{V}(x)+u_{\text {sea }}(x), d(x)=d_{V}(x)+d_{\text {sea }}(x)$. In the high $x$ limit, where there is essentially no sea quark contribution so that $R_{s} \approx 0, R_{v} \approx 1$, the parity violating asymmetry is related to $\sin ^{2} \theta_{w}$ through [4]

$$
A_{P V}^{D I S}=(109 \mathrm{ppm}) Q^{2}\left[\left(-\frac{3}{2}+\frac{10}{3} \sin ^{2} \theta_{w}\right)+Y R_{v}\left(-\frac{3}{2}+6 \sin ^{2} \theta_{w}\right)\right] .
$$

The running conditions proposed for the DIS-Parity experiment include an 11 $\mathrm{GeV}, 90 \mu \mathrm{A}$ and $80 \%$ polarized electron beam scattering off a $60 \mathrm{~cm}$ liquid deuterium target. The selected scattering angle will be $12.5^{\circ}$, corresponding to $\langle x\rangle=0.28$, $<Y>=0.62$ and $<Q^{2}>=2.9(\mathrm{GeV} / \mathrm{c})^{2}$. Under such conditions, the uncertainty on the measurement of the parity-violating asymmetry is expected to be $\delta\left(A_{P V}^{D I S}\right)=1.3 \%$, which translates into $\delta\left(\sin ^{2} \theta_{w}\right)=0.67 \%$.

While the E158 experiment is purely leptonic and not sensitive to quarks, and the $Q_{\text {weak }}$ experiment is semi-leptonic and sensitive to the $C_{1 q}$ weak quark coupling, the DIS-Parity experiment will be sensitive to the $C_{2 q}$ weak quark coupling. The experiment will perform a measurement of $C_{2 u}-\frac{1}{2} C_{2 d}$ at a precision of $\delta\left(C_{2 u}-\frac{1}{2} C_{2 d}\right)=$ 0.02. Since the weak couplings probed by the DIS-Parity experiment will be different from the ones probed by the E158 and $Q_{\text {weak }}$ experiments, the DIS-Parity will offer unique sensitivity to new physics. What makes the DIS-Parity measurement of $\sin ^{2} \theta_{w}$ particularly interesting is that the energy scale is the same as that of the $\mathrm{NuTeV}$ experiment, thus testing the $3 \sigma$ deviation from the Standard Model of the $\mathrm{NuTeV}$ 
result.

Currently under construction, the Large Hadron Collider (LHC) at the European Organization for Nuclear Research (CERN) is expected to begin running in 2007. Inside the $4.3 \mathrm{~km}$ radius $\mathrm{LHC}$ ring, two $7 \mathrm{TeV}$ counter-rotating proton beams will collide with each other, reaching a luminosity of $\sim 10^{34} \mathrm{~cm}^{-2} \mathrm{~s}^{-1}$. Several experiments are planned to take place at the LHC. The two primary ones are the CMS [57] and the ATLAS [56] experiments. The CMS experiment will make use of a 12500 ton general purpose proton-proton detector, optimized to detect the Higgs boson at the $90 \mathrm{GeV}$ to $1 \mathrm{TeV}$ range. The fields produced by the CMS solenoid magnet - the largest ever built - will reach up to 4 Tesla. The ATLAS experiment will conduct precision measurements of Standard Model parameters using a 7000 ton detector. Although roughly half the weight, the ATLAS detector is twice the size of the CMS detector and the fields inside will reach up to 2 Tesla.

One of the numerous interactions detected by the ATLAS detector will be the dilepton production near the $Z^{0}$ pole: $p p \rightarrow\left(\gamma^{*}, Z\right) \rightarrow\left(\mu^{+} \mu^{-}, e^{+} e^{-}\right)$. Such interaction exhibits a forward-backward asymmetry [6]:

$$
A_{F B}=\frac{\sigma(\cos \Theta>0)-\sigma(\cos \Theta<0)}{\sigma(\cos \Theta>0)+\sigma(\cos \Theta<0)},
$$

where $\Theta$ is the scattering angle in the lab frame. The forward-backward asymmetry is related to the effective weak mixing angle through [52]

$$
A_{F B}=b\left(a-\sin ^{2} \theta_{w}^{e f f}\left(m_{Z}\right)\right) .
$$

Parameters $a$ and $b$ depend on the rapidity ${ }^{1}$ and have been calculated for the ATLAS detector rapidity coverage $(|y|<2.5)$ to next-to-leading order in QED and QCD [37].

Given a luminosity of $100 \mathrm{fb}^{-1}$, the ATLAS experiment will be able to perform a measurement of the forward-backward asymmetry to a precision of $\delta\left(A_{F B}\right)=$ $2.3 \times 10^{-4}$, and of the weak mixing angle to a precision of $\delta\left(\sin ^{2} \theta_{w}^{e f f}\right)=1.4 \times 10^{-4}$.

\footnotetext{
${ }^{1}$ Rapidity is one of the parameters used to parametrize the quark momenta in proton-proton collisions [47].
} 
Combined with other measurements from the LHC, it will explore a new frontier of physics at the highest energies and should provide a definite test of the Higgs sector predicted in the Standard Model.

\subsection{Conclusions}

SLAC Experiment E158 provides the first direct observation of parity violation in Møller scattering. The left-right parity-violating asymmetry measured over the 2002 data collection period is

$$
\left.\left.A_{P V}=(-160 \pm 21 \text { (stat. }) \pm 17 \text { (syst. }\right)\right) \times 10^{-9}
$$

The measurement is the most precise determination of the asymmetry in electron scattering to date. The weak charge and weak mixing angle at $Q^{2}=0.026(\mathrm{GeV} / \mathrm{c})^{2}$, obtained from the measured $A_{P V}$ are

$$
\begin{aligned}
Q_{w}^{e} & =-0.048 \pm 0.006 \text { (stat.) } \pm 0.005 \text { (syst.) } \\
\sin ^{2} \theta_{w \overline{M S}} & =0.2379 \pm 0.0016 \text { (stat.) } \pm 0.0013 \text { (syst.) }
\end{aligned}
$$

which correspond to a weak mixing angle at the $Z^{0}$ pole of

$$
\sin ^{2} \theta_{w}\left(m_{Z}\right)_{\overline{M S}}=0.2306 \pm 0.0016 \text { (stat.) } \pm 0.0013 \text { (syst.) } \pm 0.0006 \text { (theory) }
$$

The E158 results are consistent with the Standard Model and provide evidence for the running of $\sin ^{2} \theta_{w}$ versus momentum transfer $Q$. Given the precision of $\delta\left(\sin ^{2} \theta_{w}\right) \sim 0.0020$, the E158 measurement of the weak mixing angle allows for the exploration of new physics effects at the $\sim \mathrm{TeV}$ level, complementing studies from collider experiments. The measurement of parity-violating asymmetries with high precision has become an important tool for studying new physics beyond the Standard Model. The success of the E158 experiment establishes that it is possible to measure asymmetries to an accuracy of $\sim 10^{-8}$ in high energy electron scattering. 


\section{Bibliography}

[1] Pre-Conceptual Design Report (pCDR) for the Science and Experimental Equipment for The $12 \mathrm{GeV}$ Upgrade of CEBAF. Draft 12.1, June 2003.

[2] P. L. Anthony et al. (SLAC E158 Collaboration). Observation of Parity Nonconservation in Møller Scattering. hep-ex/0312035, December 2003.

[3] D. Armstrong et al. The Q(Weak) Experiment: A Search for Physics at the $\mathrm{TeV}$ Scale Via a Measurement of the Proton's Weak Charge. Jlab proposal PR02-020, December 2001.

[4] J. Arrington et al. $\vec{e}-{ }^{2} H$ Parity Violating Deep Inelastic Scattering at CEBAF $6 \mathrm{GeV}$. Jlab proposal LOI-03-106, May 2003.

[5] C. Arroyo. EP Inelastic Scattering Asymmetry Correction. E158 Technical Note, TN \#33, 2003.

[6] U. Baur, S. Keller, and W. K. Sakumoto. QED Radiative Corrections to Z Boson Production and the Forward-Backward Asymmetry at Hadron Colliders. Physical Review D, 57:199-215, January 1998.

[7] S. C. Bennett and C. E. Wieman. Measurement of the $6 \mathrm{~S} \rightarrow 7 \mathrm{~S}$ Transition Polarizability in Atomic Cesium and an Improved Test of the Standard Model. Physical Review Letters, 82:2484-2487, March 1999.

[8] P. Bosted. Radiative Corrections for E158. E158 Technical Note, TN \#23, 2002.

[9] M. Breuer, Decowski P., Hicks R., and Celli J. E158 Motion Control System. E158 Technical Note, 2002.

[10] R. Carr et al. A Precision Measurement of the Weak Mixing Angle in Møller Scattering. SLAC-Proposal-E-158, July 1997. 
[11] E. Chudakov. New Detector for Polarimetry. E158 Technical Note, 2002.

[12] E. Chudakov. Polarimetry, Run I. E158 Technical Note, TN \#43, 2003.

[13] M. Cooke. Probing Moller Detector Linearity with the Polarized Iron Foil and Light Filters. E158 Technical Note, TN \#48, 2003.

[14] A. Czarnecki and W. J. Marciano. Electroweak Radiative Corrections to Polarized Møller Scattering Asymmetries. Physical Review D, 53:1066-1072, February 1996.

[15] S. Davidson, S. Forte, P. Gambino, N. Rius, and A. Strumia. Old and New Physics Interpretations of the NuTeV Anomaly. Journal of High Energy Physics, 2:37, February 2002.

[16] A. Derevianko. Reconciliation of the Measurement of Parity Nonconservation in Cs with the Standard Model. Physical Review Letters, 85:1618-1621, August 2000.

[17] E. Derman and W. J. Marciano. Parity Violating Asymmetries in Polarized Electron Scattering. Annals of Physics, 121:147, 1979.

[18] K. P. O. Diener, S. Dittmaier, and W. Hollik. Electroweak Radiative Corrections to Deep-inelastic Neutrino Scattering - Implications for NuTeV? hepph/0310364, October 2003.

[19] E. J. Eichten, K. D. Lane, and M. E. Peskin. New Tests for Quark and Lepton Substructure. Physical Review Letters, 50:811-814, March 1983.

[20] W. Emam. Dithering Analysis. E158 Technical Note, TN \#32, 2003.

[21] P. Gambino and A. Sirlin. Relation Between $\sin ^{2} \theta_{W}\left(m_{Z}\right)$ and $\sin ^{2} \theta_{\text {eff }}^{\text {lept }}$. Physical Review D, 49:1160, February 1994.

[22] J. Gao et al. A Liquid Hydrogen Target for the Precision Measurement of the Weak Mixing Angle in Møller Scattering at SLAC. Nuclear Instruments and Methods in Physics Research A, 498:90-100, February 2003. 
[23] S. L. Glashow. Partial-Symmetries of Weak Interactions. Nuclear Physics, 22:579, 1961.

[24] S. Godfrey. Comparison of Discovery Limits for Extra Z Bosons at Future Colliders. Physical Review D, 51:1402-1405, February 1995.

[25] B. Grinstein and M. B. Wise. Operator Analysis for Precision Electroweak Physics. Physics Letters B, 265:326-334, August 1991.

[26] J. F. Gunion, J. Grifols, A. Mendez, B. Kayser, and F. Olness. Higgs Bosons in Left-Right-Symmetric Models. Physical Review D, 40:1546-1561, September 1989.

[27] K. Hagiwara et al. Review of Particle Properties. Physical Review D, 66:010001, July 2002.

[28] HAPPEX Collaboration. New Measurement of Parity Violation in Elastic Electron-Proton Scattering and Implications for Strange Form Factors. Physics Letters B, 509:211-216, June 2001.

[29] F. J. Hasert at al. Observation of Neutrino-like Interactions Without Muon or Electron in the Gargamelle Neutrino Experiment. Nuclear Physics B, 73, April 1974.

[30] J. L. Hewett, T. Takeuchi, and S. Thomas. Indirect Probes of New Physics. SLAC-PUB-7088, March 1996.

[31] P. Horowitz and W. Hill. The Art of Electronics, pages 437-442. Cambridge University Press, Cambridge, 1980.

[32] T. B. Humensky, R. Alley, A. Brachmann, M. J. Browne, and J. Clendenin. SLAC's Polarized Electron Source Laser System and Minimization of Electron Beam Helicity Correlations for the E-158 Parity Violation Experiment. NASA STI/Recon Technical Report N, 3:6712, September 2002.

[33] M. Jones. Luminosity Monitor, April-June Run of E158. E158 Technical Note, TN \#21, 2001. 
[34] M. Jones. Lumi Asymmetry Analysis, All Data Runs. E158 Technical Note, TN \#54, 2003.

[35] L. Kaufman and K. Kumar. Neutral Backgrounds in the Moller Detector. E158 Technical Note, TN \#44, 2003.

[36] Y. Kolomensky. Higher Order Asymmetry Systematics. E158 Technical Note, TN \#56, 2003.

[37] S. Krzysztof, S. Riley, and U. Baur. Measurement of the Z Forward-Backward Asymmetry with the ATLAS Detector and Determination of $\sin ^{2} \theta_{\text {eff }}^{\text {lep }}\left(M_{Z}^{2}\right)$. ATL-PHYS-2000-018, May 2000.

[38] K. Kumar. Update on Neutral Backgrounds. E158 Technical Note, TN \#55, 2003.

[39] D. Lhuillier. Pion Detector Analysis. E158 Technical Note, TN \#42, 2003.

[40] I. Maksymyk, C. P. Burgess, and D. London. Beyond S, T, and U. Physical Review D, 50:529-535, July 1994.

[41] W. J. Marciano and A. Sirlin. Precise SU(5) Predictions for $\sin ^{2} \theta_{W}^{e x p}, m_{W}$, and $m_{Z}$. Physical Review Letters, 46:163-166, January 1981.

[42] T. Maruyama et al. A Very High Charge, High Polarization Gradient-Doped Strained GaAs Photocathode. Nuclear Instruments and Methods in Physics Research A, 492:199-211, October 2002.

[43] P. Mastromarino. Beam Asymmetry Analysis for E-158 Run 1. E158 Technical Note, TN \#34, 2003.

[44] G. A. Miller and A. W. Thomas. Comment on "A Precise Determination of Electroweak Parameters in Neutrino-Nucleon Scattering". hep-ex/020400\%, September 2002.

[45] R. B. Neal. The Stanford Two-Mile Accelerator, pages 652-654. W.A. Benjamin, Inc., New York, New York, 1968. 
[46] R. B. Neal. The Stanford Two-Mile Accelerator, page 307. W.A. Benjamin, Inc., New York, New York, 1968.

[47] M. Peskin and D. Schroeder. An Introduction to Quantum Field Theory, pages 565-566. Addison-Wesley Publishing Company, 1997.

[48] M. E. Peskin and T. Takeuchi. Estimation of Oblique Electroweak Corrections. Physical Review D, 46:381-409, July 1992.

[49] C. Y. Prescott et al. Parity Nonconservation in Inelastic Elctron Scattering. Physics Letters B, 77:347-352, August 1978.

[50] C. Y. Prescott et al. Further Measurements of Parity Nonconservation in Inelastic Electron Scattering. Physics Letters B, 84:524-528, July 1979.

[51] D. Relyea. A Precision Measurement of Parity Violation in Møller Scattering. Princeton University doctoral dissertation, 2003.

[52] J. L. Rosner. Off-Peak Lepton Asymmetries from New Z's. Physical Review D, 35:2244-2247, April 1987.

[53] A. Salam. Weak and electromagnetic interactions. In Elementary Particle Theory, page 367. Stockholm:Almquist and Wiksell, 1968.

[54] B. Schrempp, F. Schremmp, N. Wermes, and D. Zeppenfeld. Bounds on New Contact Interactions from Future $e^{+} e^{-}$Colliders. Nuclear Physics B, 296, January 1988.

[55] M. L. Swartz. Limits on Doubly Charged Higgs Bosons and Lepton-Flavor Violation. Physical Review D, 40:1521-1528, September 1989.

[56] The ATLAS Collaboration. ATLAS Technical Proposal. CERN/LHCC/94-43, December 1994.

[57] The CMS Collaboration. CMS Technical Proposal. CERN/LHCC/94-38, December 1994.

[58] The LEP Collaborations. A Combination of Preliminary Electroweak Measurements and Constraints on the Standard Model. CERN-EP/2002-091 [hep- 
ex/0212036], December 2002.

[59] The NuTeV Collaboration. Precise Determination of Electroweak Parameters in Neutrino-Nucleon Scattering. Physical Review Letters, 88:091802, February 2002 .

[60] The SLD Collaboration. Improved Direct Measurement of Leptonic Coupling Asymmetries with Polarized Z Bosons. Physical Review Letters, 86:11621166, February 2001.

[61] G. t'Hooft. Renormalizable Lagrangians for Massive Yang-Mills Fields. Nuclear Physics, B35:167, 1971.

[62] G. t'Hooft. Renormalization of Massless Yang-Mills Fields. Nuclear Physics, B33:173, 1971.

[63] B.T. Tognuc and M. Woods. Report on Synchrotron Radiation Analysis. E158 Technical Note, TN \#17, 2001.

[64] B. Tweedie. The GEANT3 Simulation of E158. E158 Technical Note, TN \#37, 2003.

[65] S. Weinberg. A Model of Leptons. Physical Review Letters, 19:1264-1266, November 1967.

[66] D. H. Whittum, R. A. Erickson, Y. G. Kolomensky, M. B. Woods, and G. Yocky. Overview of Cavity Beam Position Monitors for Precision Møller Scattering Measurements. SLAC ARDB Technical Note, 134:26, 1997.

[67] D. H. Whittum and Y. Kolomensky. Analysis of an Asymmetric Resonant Cavity as a Beam Monitor. Review of Scientific Instruments, 70:2300-2313, May 1999.

[68] H. Wiedemann. Particle Accelerator Physics I, page 309. Springer-Verlag Berlin Heidelberg New York, 1999.

[69] M. Woods, R. Arnold, C. Arroyo, J. Gao, M. Jones, and P. Mastromarino. Analysis of Target Density and Spotsize Asymmetries for Run 1. E158 Technical Note, TN \#40, 2003. 
[70] I. Younus. E158 Calorimeter. E158 Technical Note, TN \#47, 2003.

[71] V. Zykunov. Bremsstrahlung Corrections to the Parity-Violating Asymmetry in Møller Scattering. Yadernaya Fizika, 66, 2003. annot. 